\title{
A QUANTUM MIRKOVIĆ-VYBORNOV ISOMORPHISM
}

\author{
BEN WEBSTER, ALEX WEEKES, AND ODED YACOBI
}

\begin{abstract}
We present a quantization of an isomorphism of Mirković and Vybornov which relates the intersection of a Slodowy slice and a nilpotent orbit closure in $\mathfrak{g l}_{N}$ to a slice between spherical Schubert varieties in the affine Grassmannian of $P G L_{n}$ (with weights encoded by the Jordan types of the nilpotent orbits). A quantization of the former variety is provided by a parabolic W-algebra and of the latter by a truncated shifted Yangian. Building on earlier work of Brundan and Kleshchev, we define an explicit isomorphism between these non-commutative algebras and show that its classical limit is a variation of the original isomorphism of Mirković and Vybornov. As a corollary, we deduce that the $\mathrm{W}$-algebra is free as a left (or right) module over its Gelfand-Tsetlin subalgebra, as conjectured by Futorny, Molev, and Ovsienko.
\end{abstract}

\section{INTRODUCTION}

In MV07a Mirković and Vybornov construct an isomorphism between slices to (spherical) Schubert varieties in the affine Grassmannian of $P G L_{n}$ on the one hand and Slodowy slices in $\mathfrak{g l}_{N}$ intersected with nilpotent orbit closures on the other. This isomorphism has important applications in geometric representation theory. To name just a few occurrences, it appears in works on the mathematical definition of the Coulomb branch associated to quiver gauge theories Nak16, the analog of the geometric Satake isomorphism for affine Kac-Moody groups [BF12], and geometric approaches to knot homologies CK08, CKL10.

These varieties each have quantizations corresponding to natural Poisson structures on them. The main aim of this paper is to show that the Mirković-Vybornov isomorphism is the classical limit of an isomorphism of these quantizations.

To be more precise, the Slodowy slice $\mathcal{S}_{e}$ through a nilpotent element $e \in \mathfrak{g l}_{N}$ is quantized by a finite $\mathrm{W}$-algebra. Finite $\mathrm{W}$-algebras have been extensively studied by Kostant, Lynch, Premet, Gan-Ginzburg, and many others (cf. GG02 and references therein). The quantization of $\mathcal{S}_{e} \cap \overline{\mathbb{O}_{e^{\prime}}}$, the intersection of $\mathcal{S}_{e}$ with the closure of the nilpotent orbit through another nilpotent $e^{\prime}$, is given by a parabolic $\mathrm{W}$-algebra [Los12,Web11]. Parabolic W-algebras are quotients of finite W-algebras.

Received by the editors October 6, 2017, and, in revised form, January 14, 2019, November 12, 2019, and December 23, 2019.

2010 Mathematics Subject Classification. Primary 16S80, 17B37, 20 C99.

This research was supported in part by Perimeter Institute for Theoretical Physics. Research at Perimeter Institute is supported by the government of Canada through the Department of Innovation, Science and Economic Development Canada and by the Province of Ontario through the Ministry of Research, Innovation and Science.

The first author was supported in part by the NSF Division of Mathematical Sciences and the Alfred P. Sloan Foundation.

The second author was supported in part by NSERC and the Ontario Ministry of Training, Colleges and Universities.

The third author was supported by the Australian Reseach Council. 
Slices to Schubert varieties in the affine Grassmannian of $P G L_{n}$ are indexed by pairs $\mu, \lambda$ of dominant coweights of $P G L_{n}$, such that $\mu \leq \lambda$ in the dominant coroot ordering. We denote the slice by $\mathrm{Gr}_{\mu}^{\bar{\lambda}}$. In KWWY14 the present authors, along with Kamnitzer, quantized $\mathrm{Gr}_{\mu}^{\bar{\lambda}}$ using algebras called truncated shifted Yangians.

The Mirković-Vybornov isomorphism is an explicit isomorphism of varieties

$$
\mathcal{S}_{e} \cap \overline{\mathbb{O}_{e^{\prime}}} \cong \operatorname{Gr}_{\mu}^{\bar{\lambda}}
$$

where $e, e^{\prime}$ are related to $\mu, \lambda$ by a certain combinatorial correspondence (cf. Sections 1.2 and 4.1). Naturally one expects that (1.1) is the classical limit of an isomorphism between the quantizations of these varieties. That is our main result.

Theorem A (Theorem 4.3, part (c)). Suppose e, $e^{\prime}$ (respectively, $\mu, \lambda$ ) is a pair of nilpotent elements (respectively, dominant coweights) which are related by the Mirković-Vybornov isomorphism (1.1). Then there is an isomorphism of filtered algebras between the parabolic $W$-algebra quantizing $\mathcal{S}_{e} \cap \overline{\mathbb{O}_{e^{\prime}}}$ and the truncated shifted Yangian quantizing $\operatorname{Gr}_{\mu}^{\bar{\lambda}}$.

One can immediately conclude from this theorem that (1.1) is an isomorphism of Poisson varieties (Corollary 4.4). Moreover, since truncated shifted Yangians are explicitly presented, this theorem provides a presentation of parabolic W-algebras in type A. This generalizes Brundan and Kleshchev's foundational work on presentations of finite $\mathrm{W}$-algebras BK08.

Our final corollary of Theorem $\mathrm{A}$ uses the recent interpretation of the truncated shifted Yangian in the setting of SUSY gauge theories. The parabolic W-algebra has a distinguished maximal commutative subalgebra, called the Gelfand-Tsetlin subalgebra. In the case where $\lambda$ is a multiple of the first fundamental weight, this agrees with the Gelfand-Tsetlin subalgebra as defined by Futorny, Molev, and Ovsienko. They conjecture that the finite $\mathrm{W}$-algebra is free as a left (or right) module over its Gelfand-Tsetlin subalgebra [FMO10, Conjecture 2]. Using Theorem A we obtain (a generalization of) this conjecture by connecting it to work of Braverman, Finkelberg, and Nakajima on the mathematical theory of Coulomb branches for $3 d \mathcal{N}=4$ gauge theories.

Corollary A (Corollary 4.6). The parabolic W-algebra is free as a left (or right) module over its Gelfand-Tsetlin subalgebra.

Remark 1.1.

(1) In MV07a, the authors consider a second family of isomorphisms, based on work of Maffei [Maf05], between Slodowy slices and type A quiver varieties. This isomorphism has already been quantized by Losev Los12, Theorem 5.3.3].

(2) When $\lambda$ is a multiple of the first fundamental weight, then $\overline{\mathbb{O}_{e^{\prime}}}$ is the nilpotent cone of $\mathfrak{g l}_{N}$. In this case, the quantization of $\mathcal{S}_{e} \cap \overline{\mathbb{O}_{e^{\prime}}}$ is a central quotient of the finite $\mathrm{W}$-algebra, and the isomorphism of Theorem $\mathrm{A}$ is a variation of Brundan and Kleshchev's theorem (using the Drinfeld presentation of the Yangian instead of the RTT presentation). Indeed Losev has speculated that Brundan and Kleshchev's presentation should be understood as a quantization of the Mirković-Vybornov isomorphism Los12, Remark 5.3.4], and Theorem $\mathrm{A}$ makes this precise. 
In order to prove Theorem $\mathrm{A}$, we need results about the highest weight theory of parabolic W-algebras and truncated shifted Yangians. Brundan and Kleshchev describe the highest weights in category $\mathcal{O}$ of a finite $\mathrm{W}$-algebra in terms of row tableaux. First we describe those highest weights which descend to the parabolic W-algebra using so-called parabolic-singular elements of the Weyl group (Theorem 3.26). These are elements which are simultaneously longest left coset for a parabolic corresponding to $\mu$ and shortest right coset representatives for a parabolic corresponding to $\lambda$. This allows for the following new description of the parabolic W-algebra:

Theorem B (Theorem 3.28). In type A, the parabolic W-algebra is the quotient of the finite $W$-algebra by the intersection of annihilators of simple modules corresponding to parabolic-singular permutations.

Now to prove Theorem $\mathrm{A}$ we first prove the desired isomorphism in the case where $\lambda$ is a multiple of the first fundamental coweight (Theorem4.3(a)). This is an explicit calculation with the Brundan-Kleshchev isomorphism, comparing different subquotients of the Yangian of $\mathfrak{s l}_{n}$ on the one hand and the Yangian of $\mathfrak{g l}_{n}$ on the other. We then use results about the highest weight theory of the truncated shifted Yangian given by Kamnitzer, Tingley, and the authors in KTWWY19a and the highest weight theory of the parabolic W-algebra from Section 3.3.5 to deduce the general result from the special case.

In Section 5.1 we introduce general "MV slices" and prove an easy but useful result that any two MV slices are Poisson isomorphic (Theorem 5.5). Recently Cautis and Kamnitzer described a variation on the classical Mirković-Vybornov isomorphism, which uses MV slices that are transposes of those used by Mirković and Vybornov (cf. Section 5.3). This isomorphism is much simpler to express in coordinates, and we prove that it is the classical limit of our quantum isomorphism.

Theorem C (Theorem4.3 part (d)). The classical limit of the quantum MirkovicVybornov isomorphism in Theorem $\mathrm{A}$ agrees with Cautis and Kamnitzer's version of the classical Mirković-Vybornov isomorphism.

1.1. Notation. Throughout this paper, we alternate between letting $\mathfrak{g}$ be any simply-laced simple complex Lie algebra and specializing to the special or general linear Lie algebra. In the beginning of every section we are careful to note which setting we are in.

In general, we let $I$ denote the nodes of the Dynkin diagram of $\mathfrak{g}$, and we write $j \sim i$ to mean $j$ and $i$ are connected in the Dynkin diagram. Since Langlands duality often appears in the context of the affine Grassmannian, we will use dual notation and denote simple coroots by $\left\{\alpha_{i}\right\}_{i \in I}$ and fundamental coweights by $\left\{\varpi_{i}\right\}_{i \in I}$ and, dually, the simple roots $\left\{\alpha_{i}^{\vee}\right\}_{i \in I}$ and fundamental weights by $\left\{\varpi_{i}^{\vee}\right\}_{i \in I}$. We let $\Delta^{+}$denote the set of positive roots of $\mathfrak{g}$. When we specialize to $\mathfrak{g}=\mathfrak{s l}_{n}$ we set $I=\{1, \ldots, n-1\}$.

All spaces considered are varieties, schemes, or ind-schemes over $\mathbb{C}$.

1.2. Combinatorial data. Let $\mathfrak{g}=\mathfrak{s l}_{n}$. Consider a pair $\lambda, \mu$ of dominant coweights for $\mathfrak{g}$ such that $\lambda \geq \mu$. Write

$$
\lambda=\sum_{i=1}^{n-1} \lambda_{i} \varpi_{n-i}, \quad \mu=\sum_{i=1}^{n-1} \mu_{i} \varpi_{n-i}, \quad \lambda-\mu=\sum_{i=1}^{n-1} m_{i} \alpha_{n-i}
$$


so that $\lambda \geq \mu$ means precisely that all $m_{i} \in \mathbb{Z}_{>0}$. (Our strange indexing conventions above are chosen to match those of [KWWY14].) Define

$$
N=\sum_{i=1}^{n-1} i \lambda_{n-i}
$$

Then $N \varpi_{1} \geq \lambda \geq \mu$. Write $N \varpi_{1}-\mu=\sum_{i} m_{i}^{\prime} \alpha_{n-i}$.

We associate a pair of partitions to the above data as follows: first, the partition $\tau \vdash N$ is defined in exponential notation by

$$
\tau=\left(1^{\lambda_{n-1}} 2^{\lambda_{n-2}} \cdots(n-1)^{\lambda_{1}}\right)^{t} .
$$

Second, consider the partition $\pi \vdash N$,

$$
\pi=\left(p_{1} \leq \cdots \leq p_{n}\right),
$$

defined by

$$
p_{1}=m_{1}^{\prime}, \quad p_{2}=m_{2}^{\prime}-m_{1}^{\prime}, \ldots, p_{n-1}=m_{n-1}^{\prime}-m_{n-2}^{\prime}, \quad p_{n}=N-m_{n-1}^{\prime} .
$$

Then $\tau \geq \pi$ with respect to the dominance order on partitions.

Remark 1.2. As a matter of convention, we will write partitions as either nonincreasing or non-decreasing as appropriate.

\section{The AfFine Grassmannian SIDE}

In this section we recall truncated shifted Yangians in type A and their connection to slices in the affine Grassmannian of $P G L_{n}$. Throughout this section $\mathfrak{g}=\mathfrak{s l}_{n}$, and we fix a pair $\lambda \geq \mu$ of dominant coweights as in Section 1.1.

2.1. Slices in the affine Grassmannian. Consider (spherical) Schubert cells $\mathrm{Gr}^{\mu}, \mathrm{Gr}^{\lambda}$ in the affine Grassmannian $\mathrm{Gr}$ for $P G L_{n}$. Our running hypothesis that $\lambda \geq \mu$ implies that $\mathrm{Gr}^{\mu} \subset \overline{\mathrm{Gr}^{\lambda}}$, and we let $\mathrm{Gr}_{\mu}^{\bar{\lambda}}$ be the slice to $\mathrm{Gr}^{\mu}$ in $\overline{\mathrm{Gr}^{\lambda}}$ at the point $t^{w_{0} \mu}$. See [KWWY14, Section 2.2] for more details and precise definitions, as well as Section 5.2 below.

$\operatorname{Gr}_{\mu}^{\bar{\lambda}}$ is an irreducible affine variety of dimension $2\left\langle\rho^{\vee}, \lambda-\mu\right\rangle=2 \sum_{i} m_{i}$. It has a $\mathbb{C}^{\times}$-action by loop rotation, which contracts it to the unique fixed point $t^{w_{0} \mu} \cdot \mathrm{Gr}_{\mu}^{\bar{\lambda}}$ admits a Poisson structure which is homogeneous of degree -1 with respect to the loop rotation, as described in [KWWY14, Section 2C].

Recall that $\mathrm{Gr}$ admits a description in terms of lattices: every point is given by a $\mathbb{C}[[t]]$-lattice in $\mathbb{C}((t))^{n}$. This is well-defined only up to multiplication by a power of $t$, but we will consistently choose representatives $\Lambda$ such that $\Lambda \subset$ $\Lambda_{0}=\mathbb{C}[[t]]^{n}$. Denote $E_{\pi}=\left\{t^{p_{i}-1} e_{i}, \ldots, t e_{i}, e_{i}: \forall i\right\}$ and $E_{p}=\left\{t^{p-1} e_{i}, \ldots, e_{i}: \forall i\right\}$, where $e_{1}, \ldots, e_{n}$ is the standard basis of $\mathbb{C}^{n}$. Explicitly, we can identify

$$
\operatorname{Gr}_{\mu}^{\overline{N \varpi_{1}}}=\left\{\begin{array}{ll} 
& \text { (a) } \Lambda \subset \Lambda_{0} \text { a } \mathbb{C}[[t]] \text {-submodule, } \\
\Lambda: & \text { (b) image of } E_{\pi} \text { gives basis of } \Lambda_{0} / \Lambda, \\
& \text { (c) } \forall i, t^{p_{i}} e_{i} \in \Lambda+E_{p_{i}} .
\end{array}\right\}
$$

Since $N \varpi_{1} \geq \lambda$, we have inclusions of closed subvarieties $\overline{\operatorname{Gr}^{\lambda}} \subset \overline{\mathrm{Gr}^{N \varpi_{1}}}$ and $\operatorname{Gr}_{\mu}^{\bar{\lambda}} \subset$ $\mathrm{Gr}_{\mu}^{\overline{N \varpi_{1}}}$. Considering multiplication by $t$ as an endomorphism of $\Lambda_{0} / \Lambda$, we can also identify

$$
\operatorname{Gr}_{\mu}^{\bar{\lambda}}=\left\{\Lambda \in \operatorname{Gr}_{\mu}^{\overline{N \varpi 1}}: t \in \operatorname{End}_{\mathbb{C}}\left(\Lambda_{0} / \Lambda\right) \quad \text { has Jordan type } \leq \tau\right\}
$$


2.2. Truncated shifted Yangians. Let $Y=Y(\mathfrak{g})$ be the Yangian of $\mathfrak{g}$. This is a filtered $\mathbb{C}$-algebra with generators $E_{\alpha}^{(r)}, F_{\alpha}^{(r)}, H_{i}^{(r)}$ for $\alpha \in \Delta^{+}, i \in I, r \in \mathbb{Z}_{>0}$, and filtration defined by $\operatorname{deg}\left(X^{(r)}\right)=r$ for any generator $X$. In fact, $Y$ is generated by the elements $E_{i}^{(r)}:=E_{\alpha_{i}}^{(r)}, F_{i}^{(r)}:=F_{\alpha_{i}}^{(r)}$, and $H_{i}^{(r)}$. For the defining relations see Theorem 3.5 in [KWWY14.

We will frequently work with the formal generating series

$$
E_{i}(u)=\sum_{r>0} E_{i}^{(r)} u^{-r}, \quad F_{i}(u)=\sum_{r>0} F_{i}^{(r)} u^{-r}, \quad H_{i}(u)=1+\sum_{r>0} H_{i}^{(r)} u^{-r} .
$$

Definition 2.1 (Definition 3.10, [KWWY14]). The shifted Yangian $Y_{\mu} \subset Y$ is the subalgebra generated by $E_{i}^{(r)}, H_{i}^{(r)}$ where $r \geq 1$ and $F_{i}^{(s)}$ where $s>\mu_{i}$.

Introduce formal variables $R_{i}^{(j)}$ where $i \in I$ and $j=1, \ldots, \lambda_{i}$, and consider the tensor product of algebras

$$
Y_{\mu}\left[R_{i}^{(j)}\right]:=Y_{\mu} \otimes_{\mathbb{C}} \mathbb{C}\left[R_{i}^{(j)}: i \in I, j=1, \ldots, \lambda_{i}\right] .
$$

Let $R_{i}(u)=\sum_{j=0}^{\lambda_{i}} R_{i}^{(j)} u^{\lambda_{i}-j}$, where we denote $R_{i}^{(0)}=1$. We define $A_{i}^{(r)} \in Y_{\mu}\left[R_{i}^{(j)}\right]$ by

$$
H_{i}(u)=r_{i}(u) \frac{\prod_{j \sim i} A_{j}\left(u-\frac{1}{2}\right)}{A_{i}(u) A_{i}(u-1)},
$$

where $A_{i}(u)=1+\sum_{r>0} A_{i}^{(r)} u^{-r}$ and

$$
r_{i}(u)=u^{-\lambda_{i}} R_{i}(u) \frac{\prod_{j \sim i}\left(1-\frac{1}{2} u^{-1}\right)^{m_{j}}}{\left(1-u^{-1}\right)^{m_{i}}} .
$$

See Section 4.1 in KWWY14 for details.

Definition 2.2 (Section 4.4, [KWWY14]). Let $I_{\mu}^{\lambda}$ be the two-sided ideal of $Y_{\mu}\left[R_{i}^{(j)}\right]$ generated by $A_{i}^{(r)}$ for $r>m_{i}$. The truncated shifted Yangian is the quotient

$$
Y_{\mu}^{\lambda}:=Y_{\mu}\left[R_{i}^{(j)}\right] / I_{\mu}^{\lambda} \text {. }
$$

The subalgebra $\Gamma_{\mu}^{\lambda} \subset Y_{\mu}^{\lambda}$ generated by the images of the elements $A_{i}^{(r)}, R_{i}^{(j)}$ is commutative. In fact, it is freely generated by these elements:

$$
\Gamma_{\mu}^{\lambda}=\mathbb{C}\left[A_{i}^{(r)}, R_{i}^{(j)}: i \in I, 1 \leq r \leq m_{i}, 1 \leq j \leq \lambda_{i}\right],
$$

as follows e.g. from Corollary 2.8 below. We call $\Gamma_{\mu}^{\lambda}$ the Gelfand-Tsetlin subalgebra of $Y_{\mu}^{\lambda}$.

Remark 2.3. In some situations, it will be more convenient to adjoin formal roots $\gamma_{i, k}$ for the polynomials $R_{i}(u)=\prod_{k=1}^{\lambda_{i}}\left(u-\frac{1}{2} \gamma_{i, k}\right)$. We denote the resulting algebra $Y_{\mu}^{\lambda}(\gamma)$. This algebra carries an action of the product of symmetric groups $\Theta=$ $\prod_{i} S_{\lambda_{i}}$, and $Y_{\mu}^{\lambda}$ is the invariant subalgebra.

Definition 2.4. A set of parameters of weight $\lambda$ is a tuple $\mathbf{R}=\left(\mathbf{R}_{i}\right)_{i \in I}$, where $\mathbf{R}_{i}$ is a multiset of $\lambda_{i}$ complex numbers. via

Given a set of parameters of weight $\lambda$, we can specialize the formal variables $R_{i}^{(j)}$

$$
R_{i}(u)=\prod_{c \in \mathbf{R}_{i}}\left(u-\frac{1}{2} c\right)
$$


We denote by $Y_{\mu}^{\lambda}(\mathbf{R})$ the corresponding specialized algebra:

$$
Y_{\mu}^{\lambda}(\mathbf{R})=Y_{\mu}^{\lambda} \otimes_{\mathbb{C}\left[R_{i}^{(j)}\right]} \mathbb{C} .
$$

Note that $\mathbf{R}$ determines the roots of the specialized polynomial $R_{i}(u)$, and as a consequence we obtain a specialization of the formal variables $R_{i}^{(j)} \mapsto \mathbb{C}$. In terms of elementary symmetric functions, we can make this explicit:

$$
R_{i}^{(j)} \mapsto(-1)^{j} e_{j}\left(\frac{1}{2} \mathbf{R}_{i}\right)
$$

This same algebra arises if we number the elements of $\mathbf{R}_{i}$ and specialize $\gamma_{i, k}$ to the corresponding values. Thus, no statement about the specializations depends on which version we use, but certain statements about the families will be cleaner for $Y_{\mu}^{\lambda}(\gamma)$.

We will denote by $\Gamma_{\mu}^{\lambda}(\mathbf{R}) \subset Y_{\mu}^{\lambda}(\mathbf{R})$ the image of $\Gamma_{\mu}^{\lambda}$, so that

$$
\Gamma_{\mu}^{\lambda}(\mathbf{R})=\mathbb{C}\left[A_{i}^{(r)}: i \in I, 1 \leq r \leq m_{i}\right] .
$$

We call $\Gamma_{\mu}^{\lambda}(\mathbf{R})$ the Gelfand-Tsetlin subalgebra of $Y_{\mu}^{\lambda}(\mathbf{R})$.

Note that $Y_{\mu}^{\lambda}(\mathbf{R})$ carries a canonical filtration, as it is a quotient of the filtered algebra $Y_{\mu}$. Similarly, with notation as in the first paragraph of this section, $Y_{\mu}^{\lambda}$ and $Y_{\mu}^{\lambda}(\gamma)$ carry filtrations defined by $\operatorname{deg}\left(X^{(r)}\right)=r$ and $\operatorname{deg}\left(R_{i}^{(j)}\right)=j$ (resp., $\operatorname{deg}\left(\gamma_{i, k}\right)=1$ ).

2.3. Relationship with functions on slices. The main result of [KMWY18] is a proof of [KWWY14, Conjecture 2.20] in the case of $\mathfrak{g}=\mathfrak{s l}_{n}$. By [KWWY14, Theorem 4.10], it follows that:

Theorem 2.5. For any choice of $\mathbf{R}$, there is an isomorphism

$$
\operatorname{gr}\left(Y_{\mu}^{\lambda}(\mathbf{R})\right) \cong \mathbb{C}\left[\operatorname{Gr}_{\mu}^{\bar{\lambda}}\right]
$$

of graded Poisson algebras.

This isomorphism is given explicitly in terms of generalized minors; see [KWWY14, Section 2A] as well as Section 5.2 below.

Remark 2.6. The generalization of this theorem to $\mathfrak{g}$ to more general type is obtained in [BFN, Appendix B].

2.4. Shifted Yangians and Coulomb branches. Braverman, Finkelberg, and Nakajima have recently developed a mathematical theory of Coulomb branches for $3 d \mathcal{N}=4$ gauge theories $[\mathrm{BFN18}$, $\mathrm{BFN}]$. For any pair $(\mathbf{G}, \mathbf{N})$ of a reductive group $\mathbf{G}$ and its representation $\mathbf{N}$ (both over $\mathbb{C}$ ), they associate a moduli space $\mathcal{R}_{\mathbf{G}, \mathbf{N}}$ carrying an action of $\mathbf{G}$ and an action of $\mathbb{C}^{\times}$by loop rotation. They then define a commutative $\operatorname{ring} \mathcal{A}(\mathbf{G}, \mathbf{N}):=H_{*}^{\mathbf{G}}\left(\mathcal{R}_{\mathbf{G}, \mathbf{N}}\right)$ via a convolution product and its deformation quantization $\mathcal{A}_{\hbar}(\mathbf{G}, \mathbf{N}):=H_{*}^{\mathbf{G} \times \mathbb{C}^{\times}}\left(\mathcal{R}_{\mathbf{G}, \mathbf{N}}\right)$. The Coulomb branch is defined as the affine scheme $\mathcal{M}_{C}(\mathbf{G}, \mathbf{N}):=\operatorname{Spec} \mathcal{A}(\mathbf{G}, \mathbf{N})$.

For us, the most relevant cases of this construction are for certain quiver gauge theories and more precisely the type $A$ cases. Letting $\mathfrak{g}=\mathfrak{s l}_{n}$ and fixing coweights $\lambda, \mu$ as in Section 1.2, we define vector spaces $W_{i}=\mathbb{C}^{\lambda_{i}}$ and $V_{i}=\mathbb{C}^{m_{i}}$ for all $1 \leq i \leq n-1$. We can then define a pair $(\mathbf{G}, \mathbf{N})$ as follows:

$$
\mathbf{G}=\prod_{i=1}^{n-1} \operatorname{GL}\left(V_{i}\right), \quad \mathbf{N}=\bigoplus_{i=1}^{n-2} \operatorname{Hom}\left(V_{i}, V_{i+1}\right) \oplus \bigoplus_{i=1}^{n-1} \operatorname{Hom}\left(W_{i}, V_{i}\right)
$$


We can also incorporate the "flavor symmetry" group $\mathbf{F}=\prod_{i=1}^{n-1} \mathrm{GL}\left(W_{i}\right)$, and define

$$
\mathcal{A}_{\hbar}(\mathbf{G}, \mathbf{N} ; \mathbf{F}):=H_{*}^{\mathbf{G} \times \mathbf{F} \times \mathbb{C}^{\times}}\left(\mathcal{R}_{\mathbf{G}, \mathbf{N}}\right) .
$$

We summarize relevant results from $[\mathrm{BFN}]$ :

Theorem 2.7 ([BFN, Theorem 3.10 and Corollary B.28]). Consider data $(\mathbf{G}, \mathbf{N})$ associated to $\lambda, \mu$ as above.

(a) There is an isomorphism of graded Poisson algebras

$$
\mathbb{C}\left[\mathrm{Gr}_{\mu}^{\bar{\lambda}}\right] \cong \mathcal{A}(\mathbf{G}, \mathbf{N})
$$

In particular, $\operatorname{Gr}_{\mu}^{\bar{\lambda}} \cong \mathcal{M}_{C}(\mathbf{G}, \mathbf{N})$ is a Coulomb branch.

(b) The above isomorphism lifts to an isomorphism of filtered algebras

$$
Y_{\mu}^{\lambda} \cong \mathcal{A}_{\hbar=1}(\mathbf{G}, \mathbf{N} ; \mathbf{F})
$$

which identifies the subalgebras $\Gamma_{\mu}^{\lambda} \cong H_{\mathbf{G} \times \mathbf{F}}^{*}(p t)$.

More precisely, in the isomorphism (b) the elements $A_{i}^{(r)}$ correspond to generators of the equivariant cohomology ring $H_{\mathrm{GL}\left(V_{i}\right)}^{*}(p t)$ and the elements $R_{i}^{(j)}$ to generators of $H_{\mathrm{GL}\left(W_{i}\right)}^{*}(p t)$. Since $\mathcal{R}_{\mathbf{G}, \mathbf{N}}$ is equivariantly formal BFN18, Section 2], it follows that $\mathcal{A}_{\hbar=1}(\mathbf{G}, \mathbf{N} ; \mathbf{F})$ is free over $H_{\mathbf{G} \times \mathbf{F}}^{*}(p t)$ as a left module (and also as a right module). Thus we deduce:

Corollary 2.8. $Y_{\mu}^{\lambda}$ is free as a left (or right) module over $\Gamma_{\mu}^{\lambda}$, and $Y_{\mu}^{\lambda}(\mathbf{R})$ is free as a left (or right) module over $\Gamma_{\mu}^{\lambda}(\mathbf{R})$.

This modest application of the theory of Coulomb branches will allow us to deduce an analogous freeness result for W-algebra; see Corollary 4.6 below. We will use this connection more intensively in further work on the representation theory of these algebras KTWWY19b, Web.

2.5. Highest weights and product monomial crystals. Consider a module $M$ over the algebra $Y_{\mu}^{\lambda}(\mathbf{R})$. We call a vector $\mathbf{1} \in M$ a highest weight vector if it generates $M$ and

$$
H_{i}^{(r)} \mathbf{1} \in \mathbb{C} \mathbf{1}, \quad E_{i}^{(r)} \mathbf{1}=0 \quad \forall i \in I, r>0 .
$$

It follows that the series $H_{i}(u)$ acts on $\mathbf{1}$ by multiplication by some series

$$
J_{i}(u)=\sum_{r \geq 0} J_{i}^{(r)} u^{-r} \in 1+u^{-1} \mathbb{C}\left[\left[u^{-1}\right]\right] .
$$

We call the tuple $J=\left(J_{i}(u)\right)_{i \in I}$ the highest weight of $M$.

Conversely, given a tuple $J=\left(J_{i}(u)\right)_{i \in I}$ of series as above, there is a universal highest weight module $M(J)$ for $Y_{\mu}^{\lambda}(\mathbf{R})$ (also called a Verma or standard module). It is generated by a highest weight vector $\mathbf{1}$ with highest weight $J$ and has a unique simple quotient $L(J)$. The collection of all tuples $J$ such that $M(J) \neq 0$ (equivalently, $L(J) \neq 0$ ) is called the set of highest weights for $Y_{\mu}^{\lambda}(\mathbf{R})$. 
2.5.1. The product monomial crystal. The highest weights of $Y_{\mu}^{\lambda}(\mathbf{R})$ can be classified in terms of the weight $\mu^{*}=-w_{0} \mu$ elements of the product monomial crystal $\mathcal{B}(\mathbf{R})$, where here $w_{0} \in S_{n}$ is the longest permutation. In this section we briefly overview $\mathcal{B}(\mathbf{R})$ and its relation to highest weights in general. We then give a combinatorial model of $\mathcal{B}(\mathbf{R})$ in type A using partitions.

Remark 2.9. In this paper we will not make use of the crystal structure on $\mathcal{B}(\mathbf{R})$. Rather, we will focus on its underlying set. We refer the reader to KTWWY19a, Section 2] for further details regarding the crystal $\mathcal{B}(\mathbf{R})$. Note that in [KTWWY19a the product monomial crystal is denoted $\mathcal{B}(\lambda, \mathbf{R})$.

$\mathcal{B}(\mathbf{R})$ is a subset of the set Laurent monomials in variables $y_{i, c}$ (the "Nakajima monomial crystal"), where $i \in I, c \in \mathbb{C}$ (although strictly speaking it is only a $\mathfrak{g}-$ crystal when the parameters $\mathbf{R}$ are "integral"; see Section 2.5.3). To define $\mathcal{B}(\mathbf{R})$, one first defines the fundamental monomial crystals $\mathcal{B}\left(y_{i, c}\right)$, corresponding to a fundamental weight $\varpi_{i}$ and parameter $c \in \mathbb{C}$. It is generated by the monomial $y_{i, c}$ by applying Kashiwara operators. For any $c \in \mathbb{C}, \mathcal{B}\left(y_{i, c}\right)$ is isomorphic to the fundamental $\mathfrak{g}$-crystal of highest weight $\varpi_{i}$.

Next, the general product monomial crystal is defined by multiplying together the elements of various fundamental crystals $\mathcal{B}\left(y_{i, c}\right)$ :

$$
\mathcal{B}(\mathbf{R})=\prod_{i \in I, c \in \mathbf{R}_{i}} \mathcal{B}\left(y_{i, c}\right):=\left\{p=\prod_{i \in I, c \in \mathbf{R}_{i}} p_{i, c}: \forall i, c, p_{i, c} \in \mathcal{B}\left(y_{i, c}\right)\right\} .
$$

Here, the product symbol does not signify Cartesian product but rather the usual product in $\mathbb{C}\left[y_{i, c}^{ \pm}\right]$.

Remark 2.10. Note that with our conventions (1.2), $\lambda=\varpi_{i}$ corresponds to $\lambda_{n-i}=1$ and $\lambda_{j}=0$ for $j \neq n-i$. In particular a corresponding set of parameters $\mathbf{R}$ consists of a singleton, namely, $\mathbf{R}_{n-i}=\{c\}$, and $\mathcal{B}(\mathbf{R})$ is isomorphic to the fundamental $\mathfrak{g}$-crystal of highest weight $\varpi_{n-i}$.

We've chosen to follow the conventions of [KWWY14, which differ from those of KTWWY19a by a diagram automorphism. We pay for this choice here, since $\mathcal{B}(\mathbf{R}) \cong \mathcal{B}\left(\lambda^{*}, \mathbf{R}\right)$, where $\mathcal{B}\left(\lambda^{*}, \mathbf{R}\right)$ is the product monomial crystal as defined in KTWWY19a. We'll gain from this choice later on, since the formulation of our main results is cleaner with this convention.

The weight of a monomial is defined as follows:

$$
\mathrm{wt}\left(\prod_{i, k} y_{i, k}^{a_{i, k}}\right)=\sum_{i, k} a_{i, k} \varpi_{i}
$$

where $i \in I, k \in \mathbb{C}$, and only finitely many of the multiplicities $a_{i, k} \in \mathbb{Z}$ are non-zero. We denote the elements of weight $\mu$ by $\mathcal{B}(\mathbf{R})_{\mu}$.

For any $i \in I, k \in \mathbb{C}$, define the monomial

$$
z_{i, k}=\frac{y_{i, k} y_{i, k+2}}{\prod_{j \sim i} y_{j, k+1}} .
$$

Any element $p \in \mathcal{B}(\mathbf{R})$ can be written in the form

$$
p=y_{\mathbf{R}} z_{\mathbf{S}}^{-1}:=\prod_{i \in I, c \in \mathbf{R}_{i}} y_{i, c} \prod_{i \in I, k \in \mathbf{S}_{i}} z_{i, k}^{-1}
$$

for a unique tuple of multisets $\mathbf{S}=\left(\mathbf{S}_{i}\right)_{i \in I}$ (where products are taken with multiplicity). See Section 2 of KTWWY19a for more details. 
2.5.2. Connection to highest weights. As described in [KTWWY19a, Section 3.6], elements of $\mathcal{B}(\mathbf{R})_{\mu^{*}}$ correspond to highest weights for $Y_{\mu}^{\lambda}(\mathbf{R})$. More precisely, a monomial $p=\prod_{i, k} y_{i, k}^{a_{i, k}}$ corresponds to the series

$$
J_{i}(u):=u^{-\mu_{i}} \prod_{k}\left(u-\frac{1}{2} k\right)^{a_{i, k}}
$$

where the rational function on the right-hand side is expanded as an element of $1+u^{-1} \mathbb{C}\left[\left[u^{-1}\right]\right]$.

Theorem 2.11 ([KTWWY19a, Theorem 1.3]). The correspondence (2.12) defines a bijection between $\mathcal{B}(\mathbf{R})_{\mu^{*}}$ and the set of highest weights for $Y_{\mu}^{\lambda}(\mathbf{R})$.

Remark 2.12. In KTWWY19b we show that this theorem holds in much greater generality, using a presentation for the Yangian based on its connection to Coulomb branches, as described in $[\mathrm{BFN}$.

We note that if we write $p=y_{\mathbf{R}} z_{\mathbf{S}}^{-1}$, the tuple of multisets $\mathbf{S}=\left(\mathbf{S}_{i}\right)_{i \in I}$ encodes the action of the elements $A_{i}^{(r)} \in Y_{\mu}^{\lambda}(\mathbf{R})$ on a highest weight vector $\mathbf{1}$ of weight $p$ :

$$
A_{i}(u) \mathbf{1}=\prod_{k \in \mathbf{S}_{i}}\left(1-\frac{1}{2} k u^{-1}\right) \mathbf{1} .
$$

2.5.3. Monomials and partitions. There is an alternate description of $\mathcal{B}(\mathbf{R})$ and its combinatorics in terms of tuples of Young diagrams [KTWWY19a, Section 6.2], which we'll now explain. This will be used in Section 4.3.2

We call a set of parameters $\mathbf{R}$ integral if for every $i, \mathbf{R}_{i}$ consists of integers and, moreover, the parity of the elements in $\mathbf{R}_{i}$ equals the parity of $i$. In this case, there is a $\mathfrak{g}$-crystal structure on $\mathcal{B}(\mathbf{R})$. For arbitrary $\mathbf{R}$ we can decompose each $\mathbf{R}_{i}$ into equivalence classes $\mathbf{R}_{i}=\bigcup_{\zeta \in \mathbb{C} / 2 \mathbb{Z}} \mathbf{R}_{i}(\zeta)$, where $\mathbf{R}_{i}(\zeta)=\left\{c \in \mathbf{R}_{i} \mid c-\zeta \in 2 \mathbb{Z}+i\right\}$. We let $\mathbf{R}=\bigcup_{\zeta} \mathbf{R}(\zeta)$ be the corresponding decomposition of $\mathbf{R}$.

As sets we have that $\mathcal{B}(\mathbf{R}) \cong \bigotimes_{\zeta} \mathcal{B}(\mathbf{R}(\zeta))$; we can put a $\mathfrak{g} \oplus \cdots \oplus \mathfrak{g}$-crystal structure here, with a copy of $\mathfrak{g}$ acting independently on each equivalence class $\mathcal{B}(\mathbf{R}(\zeta))$. Therefore, to describe $\mathcal{B}(\mathbf{R})$ it suffices to describe each $\mathcal{B}(\mathbf{R}(\zeta))$. Moreover, $\mathcal{B}(\mathbf{R}(\zeta)) \cong \mathcal{B}(\mathbf{R}(\zeta)-\zeta)$, and hence we can confine ourselves to the case where $\mathbf{R}$ is integral.

First let us describe the case of a fundamental crystal $\mathcal{B}\left(y_{i, c}\right)$, where $c \equiv i \bmod 2$. As a set, it is in bijection with the collection of Young diagrams which fit into an $i \times(n-i)$ box. We picture this by placing the Young diagrams in a skew-grid. The vertices of the skew-grid are labelled by pairs $(i, \ell)$, where $i \in I$ and $\ell \equiv i \bmod 2$. The $i \times(n-i)$ box is placed in the grid with its top vertex at the point $(i, c)$.

For example, if $n=7, i=3$, and $c=5$ and the Young diagram is $(4,2)$, then we have the following picture. Here we've circled the vertex $(3,5)$, the $i \times(n-i)$ box is inscribed in blue, and the Young diagram is depicted by placing 1's in its boxes: 


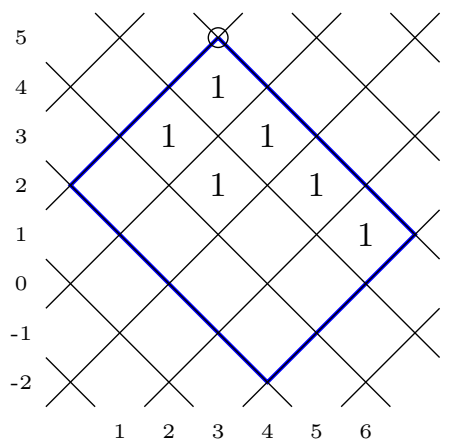

To associate a monomial to such a picture, we multiply $y_{i, c}$ by $z_{j, \ell}^{-1}$, as $(j, \ell)$ ranges over the coordinates of the bottom vertices of all the boxes in the partition. For example, the diagram above corresponds to the monomial

$$
y_{3,6} z_{3,4}^{-1} z_{2,3}^{-1} z_{4,3}^{-1} z_{3,2}^{-1} z_{5,2}^{-1} z_{6,1}^{-1} \in \mathcal{B}\left(y_{3,6}\right) \text {. }
$$

The rest of the elements of $\mathcal{B}\left(y_{3,6}\right)$ correspond to the other partitions fitting into the blue box.

In general suppose $\mathbf{R}$ is any integral set of parameters. Then elements of $\mathcal{B}(\mathbf{R})$ are identified with diagrams consisting of circled vertices and numbered boxes. The circled vertices correspond to the elements of $\mathbf{R}$ : for every $c \in \mathbf{R}_{i}$ we circle the vertex at $(i, c)$. If $c \in \mathbf{R}_{i}$ occurs with multiplicity, then the vertex is circled multiple times.

Such a diagram corresponds to an element of $\mathcal{B}(\mathbf{R})$ if and only if it can be decomposed into a tuple of overlayed partitions. More precisely, we must be able to place partitions at each circled vertex on the grid in such a way that the number in a given box counts the times that box appears in a partition. Note that a choice of such partitions may not be unique.

For example, consider the case where $\mathfrak{g}=\mathfrak{s l}_{9}$ and we take $\mathbf{R}_{3}=\{3,5,5\}, \mathbf{R}_{5}=$ $\{5\}, \mathbf{R}_{6}=\{2,4\}$, and $\mathbf{R}_{7}=\{5\}$. The left picture below depicts a candidate element of $\mathcal{B}(\mathbf{R})$. To check that it is an element of $\mathcal{B}(\mathbf{R})$ we must be able to place partitions at the circled vertices so that the number in each box counts the number of partitions that contain it. The right picture depicts such a choice of partitions, verifying that this diagram is indeed in $\mathcal{B}(\mathbf{R})$. Note that since 5 occurs twice in $\mathbf{R}_{3}$ we are able to place two partitions at $(3,5)$ :
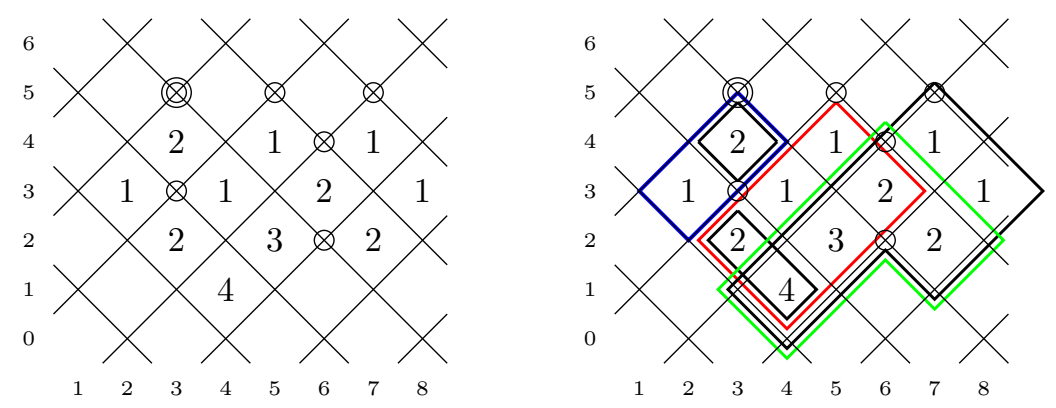

To associate a monomial to such a diagram we multiply $y_{\mathbf{R}}$ by $z_{j, l}^{-k}$, where $(j, \ell)$ ranges over the bottom vertices of the numbered boxes, and $k$ is the number of the 
box. In the example above, the diagram corresponds to the monomial

$$
y_{\mathbf{R}} z_{3,3}^{-2} z_{5,3}^{-1} z_{7,3}^{-1} z_{2,2}^{-1} z_{4,2}^{-1} z_{6,2}^{-2} z_{8,2}^{-1} z_{3,1}^{-2} z_{5,1}^{-3} z_{7,1}^{-2} z_{4,0}^{-4} \in \mathcal{B}(\mathbf{R}) .
$$

We reiterate that the assumption that $\mathbf{R}$ is an integral set of parameters is made only for the sake of convenience. We could set up the same combinatorics for general $\mathbf{R}$, where we depict elements of $\mathcal{B}(\mathbf{R})$ by tuples of such diagrams, one for each $\zeta \in \mathbb{C} / 2 \mathbb{Z}$ such that $\mathbf{R}(\zeta)$ is non-empty.

2.6. Maps between truncated shifted Yangians. Given $\lambda \geq \mu$, recall that we define $N=\sum_{i} i \lambda_{n-i}$. Consider a set of parameters $\mathbf{R}=\left(\mathbf{R}_{i}\right)_{i \in I}$ of weight $\lambda$ and a set of parameters $\widetilde{\mathbf{R}}$ of weight $N \varpi_{1}$. Note that the latter is prescribed by the single multiset $\widetilde{\mathbf{R}}_{n-1}$ of size $N$. For this reason, we will abuse our notation and simply identify $\widetilde{\mathbf{R}}=\widetilde{\mathbf{R}}_{n-1}$.

Our goal is to establish the following commutative diagram:

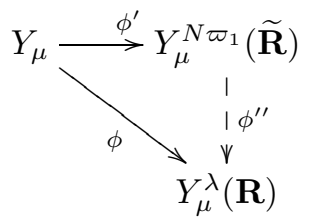

where $\phi, \phi^{\prime}$ are the (defining) quotient maps. Note that $\phi^{\prime \prime}$ is automatically filtered, if it exists, since $Y_{\mu}^{\lambda}(\mathbf{R})$ and $Y_{\mu}^{N \varpi_{1}}(\widetilde{\mathbf{R}})$ have the quotient filtration from $Y_{\mu}$.

Theorem 2.13. A map $\phi^{\prime \prime}$ making the above diagram commute exists iff

$$
\widetilde{\mathbf{R}}=\bigcup_{i=1}^{n-1}\left(\mathbf{R}_{i}+(n-i-1)\right) \cup\left(\mathbf{R}_{i}+(n-i-3)\right) \cup \cdots \cup\left(\mathbf{R}_{i}-(n-i-1)\right)
$$

as a union of multisets. In this case, $\phi^{\prime \prime}$ quantizes the inclusion $\mathrm{Gr}_{\mu}^{\bar{\lambda}} \subset \mathrm{Gr}_{\mu}^{\overline{N \varpi_{1}}}$ as a closed Poisson subvariety, and $\phi^{\prime \prime}$ maps the subalgebra $\Gamma_{\mu}^{N \omega_{1}}$ surjectively to $\Gamma_{\mu}^{\lambda}$.

The claim that $\phi^{\prime \prime}$ quantizes the inclusion simply follows from the form of the identification $\operatorname{gr} Y_{\mu}^{\lambda}(\mathbf{R}) \cong \mathbb{C}\left[\mathrm{Gr}_{\mu}^{\bar{\lambda}}\right]$. Indeed as in [KWWY14, for any $\lambda \geq \mu$ the surjection $Y_{\mu} \rightarrow Y_{\mu}^{\lambda}(\mathbf{R})$ corresponds to the inclusion $\mathrm{Gr}_{\mu}^{\bar{\lambda}} \subset \mathrm{Gr}_{\mu}$ into the opposite cell $\mathrm{Gr}_{\mu}$. So (2.14) expresses the inclusions

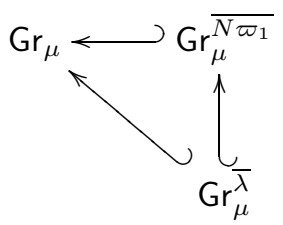

We will prove Theorem 2.13 in Section 2.6 .1 below. First we record some consequences.

When the map $\phi^{\prime \prime}$ exists, every highest weight module for $Y_{\mu}^{\lambda}(\mathbf{R})$ pulls back to a highest weight module for $Y_{\mu}^{N \varpi_{1}}(\widetilde{\mathbf{R}})$. Recall from Section 2.5 .2 that an element of the monomial crystal, expressed in the variables $y_{i, k}$, explicitly encodes the action of the series $H_{i}(u)$ on a highest weight vector. Since $H_{i}(u) \mapsto H_{i}(u)$ under $Y_{\mu}^{N \varpi_{1}}(\widetilde{\mathbf{R}}) \rightarrow Y_{\mu}^{\lambda}(\mathbf{R})$, the pull-back of highest weights corresponds to an inclusion of sets $\mathcal{B}(\mathbf{R})_{\mu^{*}} \subset \mathcal{B}(\widetilde{\mathbf{R}})_{\mu^{*}}$. Slightly more generally, we have: 
Lemma 2.14. Let $\mathbf{R}, \widetilde{\mathbf{R}}$ satisfy (2.15). Then there is an inclusion of sets

$$
\mathcal{B}(\mathbf{R}) \subset \mathcal{B}(\widetilde{\mathbf{R}}) .
$$

If $\mathbf{R}$ is integral, then this is an inclusion of crystals.

Proof. The case where $\lambda=\varpi_{i}$ is analogous to [KTWWY19a, Lemma 5.31], and the general case follows by taking products.

Remark 2.15. The above results are analogs of the embedding of $\mathfrak{s l}_{n}$ representations

$$
\left(\mathbb{C}^{n}\right)^{\otimes \lambda_{1}} \otimes\left(\wedge^{2} \mathbb{C}^{n}\right)^{\otimes \lambda_{2}} \otimes \cdots \otimes\left(\wedge^{n-1} \mathbb{C}^{n}\right)^{\otimes \lambda_{n-1}} \subset\left(\wedge^{n-1} \mathbb{C}^{n}\right)^{\otimes N},
$$

and in fact when $\mathbf{R}$ is sufficiently generic Lemma 2.14 can be interpreted as a crystal version of this embedding.

Corollary 2.16. When $\phi^{\prime \prime}: Y_{\mu}^{N \varpi_{1}}(\widetilde{\mathbf{R}}) \rightarrow Y_{\mu}^{\lambda}(\mathbf{R})$ as above exists, we have a containment

$$
\operatorname{ker} \phi^{\prime \prime} \subset \bigcap_{p} \operatorname{Ann} L_{p}
$$

the intersection being over the simple $Y_{\mu}^{N \varpi_{1}}(\widetilde{\mathbf{R}})-$ modules $L_{p}$ with highest weights $p \in \mathcal{B}(\mathbf{R})_{\mu^{*}} \subset \mathcal{B}(\widetilde{\mathbf{R}})_{\mu^{*}}$.

Defining this map in the case where we consider $R_{i}(u)$ as a formal polynomial, rather than specializing to numerical values, is slightly more complicated. Of course, Theorem 2.13 shows that we have a homomorphism $Y_{\mu}^{N \omega_{1}} \rightarrow Y_{\mu}^{\lambda}$ sending

$$
\tilde{R}_{n-1}(u) \mapsto \prod_{i=1}^{n-1} \prod_{k=1}^{n-i} R_{i}\left(u-\frac{n-i-1}{2}+k-1\right) .
$$

Unfortunately, this map is not necessarily surjective; it is more convenient to consider the enlarged version where we have a surjective map

$$
Y_{\mu}^{N \omega_{1}}(\tilde{\gamma}) \rightarrow Y_{\mu}^{\lambda}(\gamma)
$$

of the algebras from Remark 2.3. This map is defined by sending the roots of the LHS of (2.17) to the roots of the RHS (by an arbitrary bijection).

2.6.1. Proof of Theorem 2.13. Recall that we set

$$
\lambda-\mu=\sum_{i} m_{i} \alpha_{n-i}, \quad N \varpi_{1}-\mu=\sum_{i} m_{i}^{\prime} \alpha_{n-i} .
$$

In addition denote $N \varpi_{1}-\lambda=\sum_{i} m_{i}^{\prime \prime} \alpha_{n-i}$. In particular $m_{i}=m_{i}^{\prime}-m_{i}^{\prime \prime}$. We note the following:

\section{Lemma 2.17.}

$$
N \varpi_{1}-\lambda=\sum_{i=2}^{n-1} \lambda_{n-i}\left((i-1) \alpha_{1}+(i-2) \alpha_{2}+\cdots+\alpha_{i-1}\right) .
$$

Thus, we have that the coefficient $m_{1}^{\prime \prime}=0$.

Proof. We have $N \varpi_{1}-\lambda=\sum_{i} \lambda_{n-i}\left(i \varpi_{1}-\varpi_{i}\right)$. Now observe that

$$
i \varpi_{1}-\varpi_{i}=(i-1) \alpha_{1}+(i-2) \alpha_{2}+\cdots+\alpha_{i-1} .
$$


Recall from Section 2.2 that $Y_{\mu}^{\lambda}(\mathbf{R})=Y_{\mu} /\left\langle A_{i}^{(r)}: i \in I, r>m_{i}\right\rangle$, where $A_{i}^{(r)} \in Y_{\mu}$ are defined by

$$
H_{i}(u)=r_{i}(u) \frac{A_{i-1}\left(u-\frac{1}{2}\right) A_{i+1}\left(u-\frac{1}{2}\right)}{A_{i}(u) A_{i}(u-1)}
$$

with

$$
r_{i}(u)=\frac{R_{i}(u)}{u^{\lambda_{i}}} \frac{\left(1-\frac{1}{2} u^{-1}\right)^{m_{i-1}+m_{i+1}}}{\left(1-u^{-1}\right)^{m_{i}}} .
$$

Above, we follow the convention that $A_{0}(u)=A_{n}(u)=1$ and $m_{0}=m_{n}=0$.

Similarly $Y_{\mu}^{N \varpi_{1}}(\widetilde{\mathbf{R}})=Y_{\mu} /\left\langle\widetilde{A}_{i}^{(r)}: i \in I, r>m_{i}^{\prime}\right\rangle$, where $\widetilde{A}_{i}^{(r)} \in Y_{\mu}$ are defined by

$$
H_{i}(u)=\widetilde{r}_{i}(u) \frac{\widetilde{A}_{i-1}\left(u-\frac{1}{2}\right) \widetilde{A}_{i+1}\left(u-\frac{1}{2}\right)}{\widetilde{A}_{i}(u) \widetilde{A}_{i}(u-1)}
$$

where

$$
\widetilde{r}_{i}(u)=\left(\frac{\widetilde{R}(u)}{u^{N}}\right)^{\delta_{i, n-1}} \frac{\left(1-\frac{1}{2} u^{-1}\right)^{m_{i-1}^{\prime}+m_{i+1}^{\prime}}}{\left(1-u^{-1}\right)^{m_{i}^{\prime}}},
$$

with similar conventions $\widetilde{A}_{0}(u)=\widetilde{A}_{n}(0)=1$ and $m_{0}^{\prime}=m_{n}^{\prime}=0$.

From the definitions, for all $i$ we therefore have an equality in $Y_{\mu}$ :

$$
r_{i}(u) \frac{A_{i-1}\left(u-\frac{1}{2}\right) A_{i+1}\left(u-\frac{1}{2}\right)}{A_{i}(u) A_{i}(u-1)}=\widetilde{r}_{i}(u) \frac{\widetilde{A}_{i-1}\left(u-\frac{1}{2}\right) \widetilde{A}_{i+1}\left(u-\frac{1}{2}\right)}{\widetilde{A}_{i}(u) \widetilde{A}_{i}(u-1)} .
$$

Using the definition of $r_{i}(u)$ and $\widetilde{r}_{i}(u)$, for $i=n-1$ we can rewrite this as

$$
\frac{\widetilde{A}_{n-2}\left(u-\frac{1}{2}\right)}{\widetilde{A}_{n-1}(u) \widetilde{A}_{n-1}(u-1)}=\frac{R_{n-1}(u)}{\widetilde{R}(u)} \frac{u^{m_{n-1}^{\prime \prime}}(u-1)^{m_{n-1}^{\prime \prime}}}{\left(u-\frac{1}{2}\right)^{m_{n-2}^{\prime \prime}}} \frac{A_{n-2}\left(u-\frac{1}{2}\right)}{A_{n-1}(u) A_{n-1}(u-1)}
$$

and for $i=1, \ldots, n-2$ as

$$
\frac{\widetilde{A}_{i-1}\left(u-\frac{1}{2}\right) \widetilde{A}_{i+1}\left(u-\frac{1}{2}\right)}{\widetilde{A}_{i}(u) \widetilde{A}_{i}(u-1)}=R_{i}(u) \frac{u^{m_{i}^{\prime \prime}}(u-1)^{m_{i}^{\prime \prime}}}{\left(u-\frac{1}{2}\right)^{m_{i-1}^{\prime \prime}+m_{i+1}^{\prime \prime}}} \frac{A_{i-1}\left(u-\frac{1}{2}\right) A_{i+1}\left(u-\frac{1}{2}\right)}{A_{i}(u) A_{i}(u-1)} .
$$

Corollary 2.18. There are unique series $f_{i}(u) \in u^{m_{i}^{\prime \prime}}\left(1+u^{-1} \mathbb{C}\left[\left[u^{-1}\right]\right]\right)$ such that

$$
\widetilde{A}_{i}(u)=\frac{f_{i}(u)}{u^{m_{i}^{\prime \prime}}} A_{i}(u)
$$

These satisfy

$$
R_{n-1}(u)=\frac{\widetilde{R}(u) f_{n-2}\left(u-\frac{1}{2}\right)}{f_{n-1}(u) f_{n-1}(u-1)}, \quad R_{i}(u)=\frac{f_{i-1}\left(u-\frac{1}{2}\right) f_{i+1}\left(u-\frac{1}{2}\right)}{f_{i}(u) f_{i}(u-1)}
$$

for $i=1, \ldots, n-2$.

Proof. By GKLO05, Lemma 2.1], $A_{i}(u)$ and $\widetilde{A}_{i}(u)$ must differ by multiplication by an element of $1+u^{-1} \mathbb{C}\left[\left[u^{-1}\right]\right]$. The precise form above follows by rearranging (2.19) and (2.20).

Lemma 2.19. $\operatorname{ker} \phi^{\prime} \subset \operatorname{ker} \phi$ if and only if $f_{i}(u) \in \mathbb{C}[u]$. 
Proof. Assume that

$$
\widetilde{A}_{i}^{(s)} \in \operatorname{ker} \phi=\left\langle A_{i}^{(r)}: i \in I, r>m_{i}\right\rangle
$$

for all $s>m_{i}^{\prime}$. Equating coefficients in $u^{m_{i}^{\prime \prime}} \widetilde{A}_{i}(u)=f_{i}(u) A_{i}(u)$, we see that $f_{i}(u)$ cannot contain any negative powers of $u$. Indeed, if it did, then a non-trivial linear combination of elements $\left\{A_{i}^{(1)}, \ldots, A_{i}^{\left(m_{i}\right)}\right\}$ would be zero in $Y_{\mu}^{\lambda}(\mathbf{R})$. But these elements are algebraically independent in $\Gamma_{\mu}^{\lambda}(\mathbf{R})$.

Conversely, if $f_{i}(u)$ is a polynomial, then $\widetilde{A}_{i}^{(s)}$ is a linear combination of elements from ker $\phi$.

This implies that when $\phi^{\prime \prime}$ exists, it indeed sends $\Gamma_{\mu}^{N \omega_{1}}$ surjectively to $\Gamma_{\mu}^{\lambda}$. Theorem 2.13 follows from the next result:

Proposition 2.20. The map $\phi^{\prime \prime}$ exists iff the following identities hold:

$$
\begin{aligned}
\widetilde{R}(u) & =\prod_{i=1}^{n-1} R_{i}\left(u+\frac{n-i-1}{2}\right) R_{i}\left(u+\frac{n-i-3}{2}\right) \cdots R_{i}\left(u-\frac{n-i-1}{2}\right), \\
f_{k}\left(u-\frac{1}{2}\right) & =\prod_{i=1}^{k-1} R_{i}\left(u+\frac{k-i-1}{2}\right) R_{i}\left(u+\frac{k-i-3}{2}\right) \cdots R_{i}\left(u-\frac{k-i-1}{2}\right)
\end{aligned}
$$

for $k=1, \ldots, n-1$.

Proof. Note that $\phi^{\prime \prime}$ exists if and only if $\operatorname{ker} \phi^{\prime} \subset \operatorname{ker} \phi$. Hence if $\phi^{\prime \prime}$ exists, then $f_{i}(u)$ is a polynomial by Lemma 2.19, and it is monic of degree $m_{i}^{\prime \prime}$ by Corollary 2.18. Since $m_{1}^{\prime \prime}=0$ by Lemma 2.17 we know that $f_{1}(u)=1$. Applying (2.21) with $i=1$, we then obtain

$$
R_{1}(u)=f_{2}\left(u-\frac{1}{2}\right) .
$$

Proceeding by induction on $i$ using (2.21), we get the claimed form of $\widetilde{R}(u)$ and $f_{i}(u)$.

Conversely, if we define $\widetilde{R}(u)$ and $f_{i}(u)$ by the claimed form above, then (2.21) holds, and the $f_{i}(u)$ are monic polynomials of the correct degree. By the previous lemma, it follows that $\operatorname{ker} \phi^{\prime} \subset \operatorname{ker} \phi$.

\section{Around W-Algebras}

3.1. Finite $\mathbf{W}$-algebras. Let $\mathfrak{g}$ be a complex semisimple Lie algebra, and let $e \in \mathfrak{g}$ be a nilpotent element. Complete this to an $\mathfrak{s l}_{2}$-triple $\{f, h, e\}$. The Slodowy slice is the affine space $\mathcal{S}=e+\mathfrak{g}^{f}$, where $\mathfrak{g}^{f}=\{x \in \mathfrak{g} \mid[x, f]=0\}$. It naturally inherits a Poisson structure from $\mathfrak{g} \cong \mathfrak{g}^{*}$ GG02]. Recall that the symplectic leaves of $\mathfrak{g}$ are the nilpotent orbits $\mathcal{O}$, and $\mathcal{S}$ intersects the symplectic leaves transversally.

We recall now a construction of finite $\mathrm{W}$-algebras which quantize the Slodowy slices. Recall that a $\mathbb{Z}$-grading of $\mathfrak{g}$,

$$
\mathfrak{g}=\bigoplus_{i \in \mathbb{Z}} \mathfrak{g}_{i}
$$

is called good for a nilpotent $e$ if

(1) the operator $\operatorname{ad}(e)$ has degree 2 ,

(2) we have $\mathfrak{g}_{i} \cap \operatorname{ker} \operatorname{ad}(e)=0$ for $i \leq-1$,

(3) we have $\mathfrak{g}_{i} \subset$ image $\operatorname{ad}(e)$ if $i \geq 1$. 
Note that by a simple application of $\mathfrak{s l}_{2}$ representation theory, every nilpotent $e$ has a good grading induced by considering the weights of $h$.

For any good grading, the space $\mathfrak{g}_{-1}$ is symplectic with the form

$$
\langle x, y\rangle=(e,[x, y])=([e, x], y)=(x,[y, e]),
$$

where $(\cdot, \cdot)$ is the usual Killing form. This follows from the fact that $\operatorname{ad}(e): \mathfrak{g}_{-1} \rightarrow \mathfrak{g}_{1}$ is an isomorphism. Choose a Lagrangian subspace $\mathfrak{l} \subset \mathfrak{g}_{-1}$ and set

$$
\mathfrak{m}=\mathfrak{l} \oplus \bigoplus_{i<-1} \mathfrak{g}_{i}
$$

Note that if the grading in question is even (i.e., $\mathfrak{g}_{i} \neq 0$ implies $i \in 2 \mathbb{Z}$ ), then $\mathfrak{m}=\bigoplus_{i<-1} \mathfrak{g}_{i}$, and we can avoid the choice. Then $\chi=(e, \cdot): \mathfrak{m} \rightarrow \mathbb{C}$ is a character. Finally, let $\mathfrak{m}_{\chi}:=\operatorname{span}\{a-\chi(a): a \in \mathfrak{m}\}$.

Define the finite $\mathbf{W}$-algebra $W(e)=\left(U(\mathfrak{g}) / U(\mathfrak{g}) \mathfrak{m}_{\chi}\right)^{\mathfrak{m}}$. By the following theorem, this algebra is a quantization of $\mathcal{S}$.

Theorem 3.1 (Theorem 4.1, GG02). There is a filtration on $W(e)$ (the Kazhdan filtration) such that $\operatorname{gr}(W(e)) \cong \mathbb{C}[\mathcal{S}]$.

We will be interested in quotients of $W(e)$, called parabolic W-algebras, which quantize the intersection $\mathcal{S} \cap \overline{\mathcal{O}}$.

3.1.1. Conventions. We closely follow the conventions of [BK06, Section 3], BK05, Section 7], although we do not follow their grading conventions: Brundan and Kleshchev divide their even gradings by two, while we will not. We will also number the boxes of our pyramid differently. Let us briefly outline our conventions here.

For $\pi=\left(p_{1} \leq p_{2} \leq \cdots \leq p_{n}\right)$ a partition of $N$, we will consider $\pi$ as a rightjustified pyramid with boxes numbered from right to left, top to bottom. For example, $\pi=(2,3,4)$ will correspond to

$$
\begin{array}{|l|l|l|l|}
\cline { 2 - 4 } \multicolumn{1}{l|}{} & 2 & 1 \\
\cline { 2 - 4 } \multicolumn{1}{l|}{} & 5 & 4 & 3 \\
\hline 9 & 8 & 7 & 6 \\
\hline
\end{array}
$$

We number the columns of $\pi$ from left to right and rows from top to bottom.

Corresponding to the pyramid $\pi$, we consider the nilpotent element

$$
e_{\pi}=\sum_{k, \ell} e_{k \ell}
$$

summing over pairs $k \mid \ell$ of adjacent boxes in $\pi$. The grading on $\mathfrak{g}$ is defined by $\operatorname{deg}\left(e_{k \ell}\right)=2(\operatorname{col}(\ell)-\operatorname{col}(k))$, where $\operatorname{col}(\ell)$ denotes the number of the column containing $\ell$. Finally, the Kazhdan filtration on $U(\mathfrak{g})$ corresponding to $\pi$ is defined by declaring that

$$
\operatorname{deg}\left(e_{k \ell}\right)=2(\operatorname{col}(\ell)-\operatorname{col}(k)+1) .
$$

Remark 3.2. In [BK06], the authors use the convention of (3.3) in the introduction but divide by a factor of 2 in [BK06, Section 8] to match the usual filtration on Yangians. 


\subsection{Brundan and Kleshchev's presentation.}

3.2.1. Shifted Yangians. In the case where $\mathfrak{g}=\mathfrak{g l}_{N}$ Brundan and Kleshchev gave a presentation of the $\mathrm{W}$-algebra. To describe this result we first recall their definition of the shifted Yangians [BK06]. Here we work with the $\mathfrak{g l}_{n}$-Yangian $Y_{n}$, which is a $\mathbb{C}$-algebra with generators $E_{i}^{(r)}, F_{i}^{(r)}$ for $1 \leq i<n$, and $D_{i}^{(r)}$ for $1 \leq i \leq n$ and $r \geq 1$.

To describe the defining relations of $Y_{n}$ we follow BK05, Theorem 5.2] and introduce generating series $D_{i}(u)=1+\sum_{r \geq 1} D_{i}^{(r)} u^{-r}$ and define $\tilde{D}_{i}^{(r)}$ via

$$
\sum_{r \geq 0} \tilde{D}_{i}^{(r)} u^{-r}=-D_{i}(u)^{-1}
$$

The defining relations of $Y_{n}$ are as follows:

$$
\begin{aligned}
{\left[D_{i}^{(r)}, D_{j}^{(s)}\right] } & =0, \\
{\left[E_{i}^{(r)}, F_{j}^{(s)}\right] } & =\delta_{i, j} \sum_{t=0}^{r+s-1} \tilde{D}_{i}^{(t)} D_{i+1}^{(r+s-1-t)}, \\
{\left[D_{i}^{(r)}, E_{j}^{(s)}\right] } & =\left(\delta_{i, j}-\delta_{i, j+1}\right) \sum_{t=0}^{r-1} D_{i}^{(t)} E_{j}^{(r+s-1-t)}, \\
{\left[D_{i}^{(r)}, F_{j}^{(s)}\right] } & =\left(\delta_{i, j+1}-\delta_{i, j}\right) \sum_{t=0}^{r-1} F_{j}^{(r+s-1-t)} D_{i}^{(t)}, \\
{\left[E_{i}^{(r)}, E_{i}^{(s+1)}\right]-\left[E_{i}^{(r+1)}, E_{i}^{(s)}\right] } & =E_{i}^{(r)} E_{i}^{(s)}+E_{i}^{(s)} E_{i}^{(r)}, \\
{\left[F_{i}^{(r+1)}, F_{i}^{(s)}\right]-\left[F_{i}^{(r)}, F_{i}^{(s+1)}\right] } & =F_{i}^{(r)} F_{i}^{(s)}+F_{i}^{(s)} F_{i}^{(r)}, \\
{\left[E_{i}^{(r)}, E_{i+1}^{(s+1)}\right]-\left[E_{i}^{(r+1)}, E_{i+1}^{(s)}\right] } & =-E_{i}^{(r)} E_{i+1}^{(s)}, \\
{\left[F_{i}^{(r+1)}, F_{i+1}^{(s)}\right]-\left[F_{i}^{(r)}, F_{i+1}^{(s+1)}\right] } & =-F_{i+1}^{(s)} F_{i}^{(r)}, \\
{\left[F_{i}^{(r)}, F_{j}^{(s)}\right] } & =0 \quad \text { if }|i-j|>1, \\
{\left[E_{i}^{(r)},\left[E_{i}^{(s)}, E_{j}^{(t)}\right]\right]+\left[E_{i}^{(s)},\left[E_{i}^{(r)}, E_{j}^{(t)}\right]\right] } & =0 \quad \text { if }|i-j|=1, \\
{\left[F_{i}^{(r)},\left[F_{i}^{(s)}, F_{j}^{(t)}\right]\right]+\left[F_{i}^{(s)},\left[F_{i}^{(r)}, F_{j}^{(t)}\right]\right] } & =0 \quad \text { if }|i-j|=1 .
\end{aligned}
$$

$Y_{n}$ has a filtration defined as follows [BK06, Section 5]: inductively define elements $E_{i, j}^{(r)}$, for $1 \leq i<j \leq n$ and $r>0$, by $E_{i, i+1}^{(r)}=E_{i}^{(r)}$ and $E_{i, j}^{(r)}=\left[E_{i, j-1}^{(r)}, E_{j-1}^{(1)}\right]$, and similarly by $E_{i+1, i}^{(r)}=F_{i}^{(r)}$ and $E_{j, i}^{(r)}=\left[F_{j-1}^{(1)}, E_{j-1, i}^{(r)}\right]$. Also denote $E_{i, i}^{(r)}=D_{i}^{(r)}$. Then the filtration is defined by declaring the elements $E_{i, j}^{(r)}$ to have degree $r$; note that $Y_{n}$ satisfies a PBW theorem in these elements.

Let $\sigma=\left(s_{i, j}\right)_{1 \leq i, j \leq n}$ be a shift matrix of non-negative integers, meaning that

$$
s_{i, j}+s_{j, k}=s_{i, k}
$$

whenever $|i-j|+|j-k|=|i-k|$. Throughout this paper, we will use only lower-triangular shift matrices. 
Definition 3.3 (Section 2, BK06]). The shifted $\mathfrak{g l}_{\mathfrak{n}}$-Yangian $Y_{n}(\sigma) \subset Y_{n}$ is the subalgebra generated by $D_{i}^{(r)}$ for $r>0, E_{i}^{(r)}$ for $r>s_{i, i+1}$, and $F_{i}^{(r)}$ for $r>s_{i+1, i}$, with the induced filtration from $Y_{n}$.

There is another family of generators for $Y_{n}(\sigma)$, denoted $T_{i, j}^{(r)}$ for $1 \leq i, j \leq n$, and $r>s_{i, j}$. See [BK06] for the definition of these generators as well as their relation to the presentation given above. For $1 \leq i \leq n$ we define the principal quantum minor,

$$
Q_{i}(u)=\sum_{w \in S_{i}}(-1)^{w} T_{i, w(i)}(u-i+1) \cdots T_{1, w(1)}(u),
$$

where $T_{i, j}(u)=\delta_{i, j}+\sum_{r>s_{i j}} T_{i, j}^{(r)} u^{-r}$. For our present purposes, the most important relation involving these new generators is the following equation (cf. BK05, Theorem $8.7(\mathrm{i})])$ :

$$
D_{i}(u)=\frac{Q_{i}(u+i-1)}{Q_{i-1}(u+i-1)}
$$

Remark 3.4. There is a subtle point here: the identity $D_{i}(u)=\frac{Q_{i}(u+i-1)}{Q_{i-1}(u+i-1)}$ is true in the Yangian with no shift. However, in the case of an upper-triangular shift matrix, Brown and Brundan prove that the quantum minors are in fact the same, so the identity is true with the shifted $T_{i j}^{(r)}$ as well [BB09. Unfortunately, for other reasons, it is more convenient for us to use lower-triangular shift matrices, so we need to confirm that the result holds in this case as well.

Since we have to be careful about shifts, and row vs. column determinants, we include them (locally) in our notation. Define

$$
\begin{aligned}
& r Q_{i}^{\sigma}(u)=\sum_{w \in S_{i}}(-1)^{w} T_{i, w(i)}^{\sigma}(u-i+1) \cdots T_{1, w(1)}^{\sigma}(u), \\
& c Q_{i}^{\sigma}(u)=\sum_{w \in S_{i}}(-1)^{w} T_{w(1), 1}^{\sigma}(u) \cdots T_{w(i), i}^{\sigma}(u-i+1) .
\end{aligned}
$$

Let $\tau: Y_{n} \rightarrow Y_{n}$ be the transpose anti-automorphism, defined as in BK08, Section 2.3], BK05, equation (2.16)]. Then $\tau$ gives an anti-isomorphism between $Y_{n}(\sigma)$ and $Y_{n}\left(\sigma^{t}\right)$, and we note that $\tau\left(c Q_{i}^{\sigma}(u)\right)=r Q_{i}^{\sigma^{t}}(u)$.

Suppose now that $\sigma$ is a lower-triangular shift matrix, as we have assumed throughout this paper. By [BK05, equation (8.6)] we have that $\tau\left(c Q_{i}^{0}(u)\right)=$ $c Q_{i}^{0}(u)$, and by BB09, Theorem 2.2] we have that $c Q_{i}^{0}(u)=c Q_{i}^{\sigma^{t}}(u)$. Hence $c Q_{i}^{0}(u)=r Q_{i}^{\sigma}(u)$, and (3.5) also holds in our set-up.

We'll need also the decomposition

$$
Y_{n}(\sigma) \cong S Y_{n}(\sigma) \otimes Z\left(Y_{n}(\sigma)\right),
$$

where $Z\left(Y_{n}(\sigma)\right)$ is the center and $S Y_{n}(\sigma)$ is the subalgebra of $Y_{n}(\sigma)$ generated by $H_{i}^{(r)}$ for $r>0, E_{i}^{(r)}$ for $r>s_{i, i+1}$, and $F_{i}^{(r)}$ for $r>s_{i+1, i}$. Here $H_{i}^{(r)}$ are coefficients of $\frac{D_{i+1}(u)}{D_{i}(u)}$ BK08, Section 2.6]). The center $Z\left(Y_{n}(\sigma)\right)$ is freely generated by the coefficients of the series $Q_{n}(u)$ [BK08, Theorem 2.6]. 
3.2.2. Brundan and Kleshchev's theorem. Let $\pi=\left(p_{1} \leq p_{2} \leq \cdots \leq p_{n}\right)$ be a partition of $N$, and consider the lower-triangular shift matrix $\sigma$ where $s_{i, j}=p_{i}-p_{j}$ for $i \geq j$. Let $W(\pi)$ be the quotient of $Y_{n}(\sigma)$ by the two-sided ideal generated by the elements $D_{1}^{(r)}$ for $r>p_{1}$,

$$
W(\pi)=Y_{n}(\sigma) /\left\langle D_{1}^{(r)}: r>p_{1}\right\rangle
$$

The algebra $W(\pi)$ inherits a filtration from $Y_{n}(\sigma)$.

Theorem 3.5 (Theorem 10.1, BK06]). There is an isomorphism of algebras $W(\pi)$ $\cong W\left(e_{\pi}\right)$. This isomorphism doubles filtered degrees, i.e., $F_{\leq r} W(\pi) \cong F_{\leq 2 r} W\left(e_{\pi}\right)$.

We will follow the conventions of [BK08, Sections 3.3-3.4] for the above isomorphism, which differ from BK06] by a certain automorphism $\eta$. This distinction will be relevant only in Section 5.4 .

Remark 3.6. Note that the above degree doubling is harmless: the filtration (3.3) on $W\left(e_{\pi}\right)$ is even, and so we may safely rescale it removing a factor of two. This is the approach followed by Brundan and Kleshchev, so in their work no such doubling appears. We have elected to maintain the factor of two to match standard conventions on the Kazhdan filtration (e.g. GG02, Section 4]), while also following usual conventions for filtrations of Yangians.

Definition 3.7. The commutative subalgebra $\Gamma(\pi) \subset W(\pi)$ generated by the centers of the subalgebras in a chain of inclusions $W\left(\pi_{1}\right) \subset \cdots \subset W\left(\pi_{n}\right)=W(\pi)$ is called the Gelfand-Tsetlin subalgebra of $W(\pi)$, following the terminology of [FMO10.

Remark 3.8. When $\pi=(1, \ldots, 1)$, we have $W(\pi)=U\left(\mathfrak{g l}_{n}\right)$, and $\Gamma(\pi) \subset U\left(\mathfrak{g l}_{n}\right)$ is the usual Gelfand-Tsetlin subalgebra.

Consider a module $M$ over the algebra $W(\pi)$. We call a vector $\mathbf{1} \in M$ a highest weight vector if it generates $M$ and

$$
\begin{aligned}
& D_{i}^{(r)} \mathbf{1} \in \mathbb{C} \mathbf{1} \quad \text { for } i=1, \ldots, n, r \geq 1, \\
& E_{i, j}^{(r)} \mathbf{1}=0 \quad \text { for } 1 \leq i<j \leq n, r \geq 1 .
\end{aligned}
$$

As in Section 2.5, the highest weight of $M$ is a collection of series whose coefficients record the action of the $D_{i}^{(r)}$ on $\mathbf{1}$.

Let $\operatorname{Row}(\pi)$ be the set of row symmetrized $\pi$-tableaux, i.e., tableaux of shape $\pi$ with complex entries viewed up to row equivalence. A row tableau $T \in \operatorname{Row}(\pi)$ encodes a highest weight of $W(\pi)$ via

$$
(u-i+1)^{p_{i}} D_{i}(u-i+1) \mapsto \prod_{a \in T_{i}}\left(u+\frac{1}{2} a-\frac{n}{2}\right),
$$

where $T_{i}$ denotes the $i$-th row of $T$. Brundan and Kleshchev prove that this describes a bijection between highest weights of $W(\pi)$ and $R o w(\pi)$ (BK08, Section $6]$ ). Given a multiset $\mathbf{R}$ of $N$ complex numbers we let $\operatorname{Row}_{\mathbf{R}}(\pi)$ be the set of row tableaux with entries from $\mathbf{R}$ (with the same multiplicities). 
3.3. Parabolic $\mathbf{W}$-algebras. We will require some facts about parabolic Walgebras which may be of some independent interest.

In type A parabolic W-algebras quantize the intersection of a Slodowy slice with the closure of a nilpotent orbit. They arise from Hamiltonian reduction of the primitive quotients of the universal enveloping algebra. These quotients were studied by the first author Web11, Section 2] and by Losev [Los12, Section 5.2].

3.3.1. Differential operators on partial flag varieties. Let $G$ be a reductive complex algebraic group. Given a parabolic $P$, we consider the homogenous space $X=G / P$ and the universal differential operators on it as a quotient of $U(\mathfrak{g})$. Let $\mathfrak{g} \cong \mathfrak{u}-\oplus \mathfrak{l} \oplus \mathfrak{u}$ be the decomposition of $\mathfrak{g}=\operatorname{Lie}(G)$ into a Levi subalgebra, and two complementary radicals, with $\mathfrak{p}=\mathfrak{l} \oplus \mathfrak{u}$.

We'll be interested in sheaves of twisted differential operators on $X$. See BB93, Sections 1-2] for a general discussion of these rings. Since we wish to consider TDOs over more general rings, let us give a complete definition. Fix a commutative $\mathbb{C}$ algebra $S$.

Definition 3.9. A filtered sheaf of algebras $\mathscr{D}$,

$$
\{0\}=\mathscr{D}_{\leq-1} \subset \mathscr{D}_{\leq 0} \subset \cdots \subset \mathscr{D}, \quad \bigcup_{n \geq 0} \mathscr{D}_{\leq n}=\mathscr{D},
$$

is a TDO with coefficients in $S$ if there is an isomorphism of graded Poisson algebras

$$
\operatorname{gr} \mathscr{D} \rightarrow \operatorname{Sym}^{\bullet}(\mathcal{T}) \otimes S,
$$

where $\mathcal{T}=\mathcal{T}(X)$ is the tangent sheaf of $X$. The Poisson bracket on $\operatorname{Sym}^{\bullet}(\mathcal{T}) \otimes S$ is the unique $S$-linear Poisson bracket such that $\{X, Y\}$ is the Lie derivative $\mathcal{L}_{X} Y$ for $X$ a vector field and $Y$ an arbitrary tensor.

A homogeneous TDO is a TDO equipped with a $G$-equivariant structure and a Lie algebra map $\mathfrak{g} \rightarrow \Gamma\left(X ; \mathscr{D}_{\leq 1}\right)$ lifting the action map $\mathfrak{g} \rightarrow \Gamma(X ; \mathcal{T})$.

As in [BB81, we consider the sheaf of $\mathfrak{g}$-valued functions $\mathfrak{g}^{0}=\mathfrak{g} \otimes \mathcal{O}_{X}$. Note that $\mathfrak{g}^{0}$ is the sheaf of sections of the trivial bundle $X \times \mathfrak{g}$, and we have a short exact sequence of vector bundles

$$
0 \rightarrow G \times{ }_{P} \mathfrak{p} \rightarrow X \times \mathfrak{g} \rightarrow G \times_{P} \mathfrak{g} / \mathfrak{p} \rightarrow 0 .
$$

We let $\mathfrak{p}^{0}$ be the local sections of $G \times{ }_{P} \mathfrak{p}$, and so we have an exact sequence of sheaves

$$
0 \rightarrow \mathfrak{p}^{0} \rightarrow \mathfrak{g}^{0} \rightarrow \mathcal{T} \rightarrow 0 .
$$

We consider also the algebra sheaf $U^{0}=U\left(\mathfrak{g}^{0}\right) \otimes S=U(\mathfrak{g}) \otimes \mathcal{O}_{X} \otimes S$.

Given a character $\gamma: \mathfrak{p} \rightarrow S$, we consider the ideal in $\mathcal{I}^{\gamma} \subset U^{0}$ generated by the kernel of the map $U\left(\mathfrak{p}^{0}\right) \otimes S \rightarrow \mathcal{O}_{X} \otimes S$ induced by the character $\gamma-\rho+\rho_{P}: \mathfrak{p}^{0} \otimes S \rightarrow$ $\mathcal{O}_{X} \otimes S$. Here $\rho$ is the usual half-sum of positive roots of $G$, and $\rho_{P}$ is the half-sum of the positive roots of the Levi subgroup $L$. In other words $\mathcal{I}^{\gamma}$ is generated by $\xi-\left(\gamma-\rho+\rho_{P}\right)(\xi)$, where $\xi \in \mathfrak{p}^{0} \otimes S$. Define $\mathscr{D}_{\gamma}=U^{0} / \mathcal{I}^{\gamma}$.

We can define a TDO $\mathscr{D}_{\gamma}$ on $X$ by considering the quotient of $U^{0}$ by this ideal, with the obvious homogeneous structure.

Proposition 3.10 ([Mil, Theorem 2.4]). This construction defines a bijection between homogeneous TDOs on $X$ and characters $\gamma: \mathfrak{p} \rightarrow S$. 
If we choose $S=\operatorname{Sym}(\mathfrak{p} /[\mathfrak{p}, \mathfrak{p}])$, we can take the universal character $\iota: \mathfrak{p} \rightarrow$ $\mathfrak{p} /[\mathfrak{p}, \mathfrak{p}] \subset S$. We can consider the section algebra $A(\mathfrak{p})=\Gamma\left(X ; \mathscr{D}_{\iota}\right)$. When there is no risk of confusion, we will simply write $A$. We'll also consider two other cases: when $S=\mathbb{C}$ and $\gamma: \mathfrak{p} \rightarrow \mathbb{C}$ is an honest character, and when $S=\widehat{\operatorname{Sym}}(\mathfrak{p} /[\mathfrak{p}, \mathfrak{p}])$, the completion of $\operatorname{Sym}(\mathfrak{p} /[\mathfrak{p}, \mathfrak{p}])$ at 0 , and we have $\gamma+\iota: \mathfrak{p} \rightarrow \widehat{\operatorname{Sym}}(\mathfrak{p} /[\mathfrak{p}, \mathfrak{p}])$. We have the resulting algebras $A_{\gamma}(\mathfrak{p})=\Gamma\left(X ; \mathscr{D}_{\gamma}\right)$ and $A_{\gamma+\iota}(\mathfrak{p})=\Gamma\left(X ; \mathscr{D}_{\gamma+\iota}\right)$, and as above, when there is no risk of confusion we'll write simply $A_{\gamma}, A_{\gamma+\iota}$.

We always have that gr $A_{\gamma} \cong \mathbb{C}\left[T^{*} X\right]$ with the grading induced by cotangent scaling. Note that this shows that the algebra $A$ is flat over $\operatorname{Sym}(\mathfrak{p} /[\mathfrak{p}, \mathfrak{p}])$, since its fibers have constant character for the $\mathbb{C}^{\times}$-action (so actually every piece of the order filtration is flat). This shows that $A_{\gamma+\iota}$ is flat as well.

Thus, the algebra $A_{\gamma+\iota}$ provides a family over a regular ring which interpolates between the generic behavior around $\gamma$ and the specialized behavior at $\gamma$. In this case, we let $K$ be the fraction field of $S=\widehat{\operatorname{Sym}}(\mathfrak{p} /[\mathfrak{p}, \mathfrak{p}])$ and let $\tilde{\mathscr{D}}_{\gamma}:=\mathscr{D}_{\gamma+\iota} \otimes_{S} K$ denote the TDO over $S$ associated to $\gamma+\iota$, base changed to $K$. We let $\tilde{A}_{\gamma}=$ $A_{\gamma+\iota} \otimes_{S} K$.

This last algebra is interesting because it satisfies the appropriate analog of the Beilinson-Bernstein theorem for all $\gamma$, without any dominance hypothesis. This should be expected, because $\gamma$ is always "generic", but the sense in which localization holds generically is subtle, since it is not a Zariski open property. However, it is easy to check that the original proof of Beilinson-Bernstein BB81 and its extension to the parabolic case by [Kit12, 2.9] work over any characteristic 0 field, in particular over $K$. Here we must interpret "dominant" as in Kit12, Section 2.6]: a weight over $K$ is dominant if for all $i$, its inner product with $\alpha_{i}^{\vee}$ is not a negative integer. The weight $\gamma+\iota$ is obviously dominant in this sense since this inner product is never an integer 1 Thus we have that:

Theorem 3.11. The functor

$$
\Gamma(X ;-): \tilde{\mathscr{D}}_{\gamma}-\bmod \rightarrow \tilde{A}_{\gamma}-\bmod
$$

is an equivalence.

The algebra $A(\mathfrak{p})$ is not quite an analog of the universal enveloping algebra since even in the case of a Borel $\mathfrak{p}=\mathfrak{b}$, we will not obtain $U(\mathfrak{g})$, but instead the finite extension $A(\mathfrak{b})=U(\mathfrak{g}) \otimes_{Z(\mathfrak{g})} U(\mathfrak{h})$ quantizing the Grothendieck-Springer resolution.

Geometrically, we can see that $A(\mathfrak{p})$ is a quotient of $A(\mathfrak{b})$ because the pull-back $\pi^{*} \mathscr{D}_{\iota}$ by the projection map $G / B \rightarrow G / P$ is a quotient of the corresponding TDO $\mathscr{D}_{\mathfrak{l}}^{(\mathfrak{b})}$ on $G / B$, induced by the map $\mathfrak{h}=\mathfrak{b} /[\mathfrak{b}, \mathfrak{b}] \rightarrow \mathfrak{p} /[\mathfrak{p}, \mathfrak{p}]$. A $\mathscr{D}_{\iota}^{(\mathfrak{b})}$-module can be thought of as a "coherent sheaf with connection" with special curvature, and this quotient imposes the condition of flatness along fibers of the map $\pi$.

Algebraically, $A(\mathfrak{b})$ contains the subalgebra generated by $U(\mathfrak{p})$ and $U(\mathfrak{h})$, and this subalgebra has a representation on $\operatorname{Sym}(\mathfrak{p} /[\mathfrak{p}, \mathfrak{p}])$ where both map by the obvious quotients. A quick computation with the Harish-Chandra homomorphism shows these are compatible. We can algebraically express $A(\mathfrak{p})$ as the quotient of $A(\mathfrak{b})$ that acts faithfully on the induced parabolic Verma module.

\footnotetext{
${ }^{1}$ The papers BB81 and Kit12 use opposite sign conventions. Luckily, this is irrelevant for us since $\gamma+\iota$ is dominant and anti-dominant in this sense, so even if one mixes up the sign conventions, one will arrive at the correct result.
} 
When we ultimately compare parabolic $\mathrm{W}$-algebras to Yangians, this algebra matches the larger algebra $Y_{\mu}^{\lambda}(\gamma)$, where formal roots of $R_{i}$ are adjoined; see Remark 2.3 .

We can identify $Z(\mathfrak{g})$ as a subalgebra of $U(\mathfrak{h})$ in two different ways: there is the usual Harish-Chandra homomorphism, which sends a central element to the Cartan term in its PBW expansion, and the $\rho$-shifted version of this homomorphism, which identifies $Z(\mathfrak{g})$ with $U(\mathfrak{h})^{W}$, so the maximal ideal for the orbit of a weight $\lambda$ is the ideal of central elements vanishing on the Verma module of highest weight $\lambda-\rho$. We'll usually want to use the latter, but it will be useful to sometimes have the former. Note that either map will give $A(\mathfrak{b})=U(\mathfrak{g}) \otimes_{Z(\mathfrak{g})} U(\mathfrak{h})$; the question is just one of the coordinates on $\mathfrak{h}$.

Remark 3.12. Note that in the case of $\mathfrak{g l}_{N}$, this matches the convention of [BK08, Section 3.8]: the elements $Z_{N}^{(r)}$ are sent to the degree $r$ elementary symmetric function in the diagonal elements $e_{i, i}$. If we identify a dominant weight of $\mathfrak{g l}_{N}$ with a partition $\nu_{1} \geq \cdots \geq \nu_{N}$ as usual, then this shift sends it to $\left(\nu_{1}+\frac{N-1}{2}, \nu_{2}+\right.$ $\left.\frac{N-3}{2}, \ldots, \nu_{N}+\frac{1-N}{2}\right)$.

We have an induced $W$-action on $A(\mathfrak{b})=U(\mathfrak{g}) \otimes_{Z(\mathfrak{g})} U(\mathfrak{h})$ trivially on the first tensor factor and as the usual action on the second (if we use the shifted HarishChandra homomorphism). Thus, we can recover $U(\mathfrak{g})$ as the invariants of this action.

Consider the group $\Theta=N_{G}(\mathfrak{l}) / L$, the normalizer of the Levi $\mathfrak{l}$ of $\mathfrak{p}$ in $G$ modulo the Levi subgroup integrating it. Since Cartan subalgebras in $\mathfrak{l}$ are unique up to conjugacy in $L$, we have that $\Theta$ is also the simultaneous normalizer of $L$ and $H$ modulo $H$. That is, it is the subgroup of $W$ normalizing $L$.

For general $\mathfrak{p}$, we can write $\mathfrak{p} /[\mathfrak{p}, \mathfrak{p}]=\mathfrak{z}(\mathfrak{l})$ as a quotient of $\mathfrak{h}$ and thus write $A(\mathfrak{p})$ as a quotient of $A(\mathfrak{b})=U(\mathfrak{g}) \otimes_{Z(\mathfrak{g})} U(\mathfrak{h})$, where here we have to be sure to use the unshifted Harish-Chandra homomorphism (and thus act by the dot action on $\mathfrak{h}$ ). The elements of $\Theta \subset W$ descend to automorphisms of $A(\mathfrak{p})$ under this map.

Definition 3.13. Let $W(0, \mathfrak{p})=A(\mathfrak{p})^{\Theta} \subset A(\mathfrak{p})$ be the invariant subalgebra.

Remark 3.14. In the type A context of primary interest to us, the Levi $\mathfrak{l}$ will be the block diagonal matrices with block sizes given by some composition; the group $\Theta$ will be a product of symmetric groups permuting the blocks with the same size. Under our ultimate match of conventions, the scalars $\lambda_{i}$ will be the number of blocks of size $i$, so $\Theta=\prod S_{\lambda_{i}}$. Note that this matches the use of $\Theta$ in Remark 2.3.

Note that we always have a surjective map of $S=\operatorname{Sym}(\mathfrak{p} /[\mathfrak{p}, \mathfrak{p}])$-algebras $U(\mathfrak{g}) \otimes_{\mathbb{C}}$ $S \rightarrow A(\mathfrak{p})$ as proven by Borho and Brylinski [BB82, 3.8]. Since this is a surjective map, it sends the center $Z(\mathfrak{g}) \otimes_{\mathbb{C}} S$ to the center $S$ of $A(\mathfrak{p})$.

The map $Z(\mathfrak{g}) \cong U(\mathfrak{h})^{W} \rightarrow S$ is induced by the translation by $\rho_{P}$, followed by the obvious projection $\mathfrak{h} \rightarrow \mathfrak{p} /[\mathfrak{p}, \mathfrak{p}]$. That is, the induced map on spectra sends a character $\gamma$ on $\mathfrak{p}$ to the $W$-orbit of the restriction of $\gamma+\rho_{P}$ to $\mathfrak{h}$. Since the $\Theta$-action is constructed by pushing down the action of $W$ in $U(\mathfrak{g}) \otimes_{Z(\mathfrak{g})} U(\mathfrak{h})$, the image of the natural map $U(\mathfrak{g}) \rightarrow A(\mathfrak{p})$ is $\Theta$-invariant. Though the map $W(0, \mathfrak{p}) \hookrightarrow A(\mathfrak{p})$ is not surjective, it becomes so after base change to $\mathbb{C}$ :

Lemma 3.15. The algebra $A_{\gamma}(\mathfrak{p})$ is naturally isomorphic to the quotient $W(0, \mathfrak{p})_{\gamma}$ of $W(0, \mathfrak{p})$ by the maximal ideal in $Z(\mathfrak{g})$ which corresponds to the weight $\gamma+\rho_{P}$ under the Harish-Chandra homomorphism. 
Proof. We have a surjective map $U(\mathfrak{g}) \otimes_{\mathbb{C}} S \rightarrow A_{\gamma}$, sending every element of $S$ to a scalar by [BB82, 3.8], so $U(\mathfrak{g}) \rightarrow A_{\gamma}$ must be surjective, and of course this factors through the map $W(0, \mathfrak{p}) \rightarrow A_{\gamma}$. Our calculation above of the map $Z(\mathfrak{g}) \rightarrow S$ shows that the maximal ideal for the weight $\gamma+\rho_{P}$ is indeed killed by this map. That this gives all elements of the ideal is easily checked by considering the associated graded.

3.3.2. Specializing to type $A$. For $\mathfrak{g l}_{N}$, we can take the parabolic subalgebra $\mathfrak{p}$ to consist of block upper triangular matrices for some composition $\tau$ of $N$. In particular, if $\tau=\left(\tau_{1}, \ldots, \tau_{\ell}\right)$, then the Levi subalgebra $\mathfrak{l} \subset \mathfrak{p}$ comprises block diagonal matrices where the $j$ th block consists of $\tau_{j} \times \tau_{j}$ matrices. A character $\gamma: \mathfrak{p} \rightarrow \mathbb{C}$ is simply an assignment of a scalar $r_{j}$ to the $j$ th block for $j=1, \ldots, \ell$. Given $\gamma$ we define a multiset $\mathbf{R}_{i}$ to be the set of (twice) the values we assign to a block of length $i$ :

$$
\mathbf{R}_{i}=\left\{2 r_{j} \mid \tau_{j}=i, j=1, \ldots, \ell\right\} .
$$

Combining these we obtain a set of parameters $\mathbf{R}=\left(\mathbf{R}_{i}\right)_{i \in I}$ (cf. Definition 2.4). (The factor of 2 in the definition of $\mathbf{R}_{i}$ is inserted to match the conventions of Section 2)

The vector $\rho_{P}$ is given by

$$
\frac{1}{2}\left(\tau_{1}-1, \tau_{1}-3, \ldots,-\tau_{1}+1, \ldots, \tau_{\ell}-1, \tau_{\ell}-3, \ldots,-\tau_{\ell}+1\right),
$$

so the weight $\gamma+\rho_{P}$ is a concatenation of vectors of the form $\frac{1}{2}(r+i-1, r+i-$ $3, \ldots, r-i+1)$ for the different $r \in R_{i}$. The normalizer $\Theta$ acts by permuting these blocks if they have the same size (cf. Remark 3.14), so after taking the $\Theta$-invariants of $A(\mathfrak{p})$, we need only remember $\mathbf{R}$. In other words, given $\mathbf{R}$ which is compatible with $\mathfrak{p}$ (that is, $\left|\mathbf{R}_{i}\right|$ equals the number of $i \times i$ blocks in $\mathfrak{l}$ ), we can choose a $\gamma$ so that $\gamma+\rho_{P}$ recovers $\mathbf{R}$ as above. The corresponding two-sided ideal of $W(0, \mathfrak{p})$ generated by the maximal ideal of $Z\left(\mathfrak{g l}_{N}\right)$ is independent of the choice of $\gamma$. Thus, we will use $W(0, \mathfrak{p})_{\mathbf{R}}$ to denote this quotient of $W(0, \mathfrak{p})$. By Lemma 3.15 that natural map $W(0, \mathfrak{p}) \rightarrow A(\mathfrak{p})$ induces an isomorphism $W(0, \mathfrak{p})_{\mathbf{R}} \cong A_{\gamma}(\mathfrak{p})$.

Remark 3.16. If we replace $\mathrm{GL}_{N}$ with $\mathrm{SL}_{N}$, we simply kill the kernel of the surjective map $U\left(\mathfrak{g l}_{N}\right) \rightarrow U\left(\mathfrak{s l}_{N}\right)$, which means that $\mathbf{R}$ would only be well-defined up to simultaneous translation. Alternatively, we can think about this in terms of the unique automorphism of $U\left(\mathfrak{g l}_{N}\right)$ which fixes $U\left(\mathfrak{s l}_{N}\right)$ and sends $Z_{N}^{(1)} \mapsto Z_{N}^{(1)}+k$. Thus, we have $W(0, \mathfrak{p})_{\mathbf{R}} \cong W(0, \mathfrak{p})_{\mathbf{R}+k}$ for any $k \in \mathbb{C}$.

Note that if $\mathfrak{p}=\mathfrak{b}$ is a Borel, then all blocks are of size 1 so we only have $R_{1}$. We let $U(\mathfrak{g})_{\mathbf{R}}=W(0, \mathfrak{b})_{\mathbf{R}}$. As discussed above (cf. Remark 3.12), the quotient $U(\mathfrak{g})_{\mathbf{R}}$ can be defined by sending $Z_{N}^{(s)}$ to the scalar $e_{s}\left(R_{1}\right)$, that is, by sending the formal polynomial $Z_{N}(u) \mapsto \prod_{r \in R_{1}}(u+r / 2)$. Our Harish-Chandra homomorphism calculation shows that:

Lemma 3.17. The surjective map $U(\mathfrak{g}) \rightarrow W(0, \mathfrak{p})_{\mathbf{R}}$ factors through $U(\mathfrak{g})_{\widetilde{\mathbf{R}}}$ where $\widetilde{\mathbf{R}}$ satisfies the condition of (2.15).

Remark 3.18. In this formalism, we can think of the deformation $A_{\gamma+\iota}$ as corresponding to a similar set, where we replace each complex number $r \in R_{i}$ with a "point" in a formal neighborhood of this point. 
3.3.3. Definition of parabolic W-algebras. Now we consider W-algebra analogs of the algebras defined in the previous section, which will be defined by non-commutative Hamiltonian reduction. Following the notation from Section 3.1, for any module $N$ of a quotient of $U(\mathfrak{g}) \otimes_{\mathbb{C}} S$, we have an induced m-action where

$$
m \cdot n=m n-\chi(m) n \text { for all } m \in \mathfrak{m}, n \in N,
$$

where on the RHS, the action is the module structure. Let $Q_{\pi}=W(0, \mathfrak{p}) / W(0, \mathfrak{p}) \mathfrak{m}_{\chi}$ and consider the non-commutative Hamiltonian reductions

$$
\begin{aligned}
A(e, \mathfrak{p}) & :=\operatorname{Hom}_{A(\mathfrak{p})}\left(A(\mathfrak{p}) / A(\mathfrak{p}) \mathfrak{m}_{\chi}, A(\mathfrak{p}) / A(\mathfrak{p}) \mathfrak{m}_{\chi}\right)=\left(A(\mathfrak{p}) / A(\mathfrak{p}) \mathfrak{m}_{\chi}\right)^{\mathfrak{m}}, \\
W(e, \mathfrak{p}) & :=\operatorname{Hom}_{W(0, \mathfrak{p})}\left(Q_{\pi}, Q_{\pi}\right)=Q_{\pi}^{\mathfrak{m}} .
\end{aligned}
$$

The algebra $W(e, \mathfrak{p})$ is the parabolic W-algebra.

We can also obtain $W(e, \mathfrak{p})_{\gamma}, A(e, \mathfrak{p})_{\gamma+\iota}$, and $\tilde{A}(e, \mathfrak{p})_{\gamma}$ over $\mathbb{C}, \widehat{\operatorname{Sym}}(\mathfrak{p} /[\mathfrak{p}, \mathfrak{p}])$ and $K$, respectively, by tensoring $A(e)$ with the appropriate base ring or by Hamiltonian reduction of the corresponding algebras when $e=0$. The equivalence of these descriptions follows from the flatness of $A(e, \mathfrak{p})$ over $\operatorname{Sym}(\mathfrak{p} /[\mathfrak{p}, \mathfrak{p}])$ and the fact that $H^{i}\left(\mathfrak{m} ; Q_{\pi}\right)=0$ for $i>0$. This latter vanishing is proven exactly as in GG02, Proposition 5.2]; the argument there uses only that the group $M$ integrating $\mathfrak{m}$ acts freely on the coadjoint orbit through $\chi$ and thus applies to any algebra with an inner action of $\mathfrak{m}$. Note that $W(e, \mathfrak{b}) \cong W(e)$, as defined in Section 3.1. In type A, we can use the notation $W(e)_{\mathbf{R}}, W(e, \mathfrak{p})_{\mathbf{R}}$ as in Section 3.3.1 as discussed there, these algebras only depend on $\mathbf{R}$ up to simultaneous translation.

The algebra $W(e, \mathfrak{p})_{\gamma}$ comprises the sections of a quantum structure sheaf on the S3-variety $\mathfrak{X}_{\mathfrak{p}}^{e}$, as defined in BLPW16, Section 9.2]. As proven in Web11, Proposition 10] and [Los12, Lemma 5.2.1], the associated graded of this algebra is isomorphic to the algebra of global functions on $\mathfrak{X}_{\mathfrak{p}}^{e}$.

We can also write $W(e, \mathfrak{p})$ as a quotient of the finite $\mathrm{W}$-algebra $W(e) \rightarrow W(e, \mathfrak{p})$ by an ideal $J_{\mathfrak{p}}$. This ideal is constructed by considering the kernel $I_{\mathfrak{p}}$ of the map $U(\mathfrak{g}) \rightarrow W(0, \mathfrak{p})$ and then applying Losev's lower dagger operation $J_{\mathfrak{p}}:=\left(I_{\mathfrak{p}}\right)_{\dagger}$ Los10. Note that this ideal must be prime, since $W(e, \mathfrak{p})$ is a domain. Our aim is to ultimately understand this ideal, using the geometry of $X$.

Definition 3.19. The Gelfand-Tsetlin subalgebra $\Gamma(\pi, \mathfrak{p})$ of $W(\pi, \mathfrak{p})$ is the image under the natural quotient map of $\Gamma(\pi)$.

Remark 3.20. We can make a slightly cleaner statement about the classical limit of $W(e, \mathfrak{p})_{\gamma}$ if the natural map $T^{*} X \rightarrow \mathfrak{g}^{*}$ is generically injective. This is always the case in type A, but for some parabolics in other Lie algebras it fails; for the classical groups, a criterion for this property is given by Hesselink [Hes78, Theorem 7.1]. In this case, the obvious map induces an isomorphism $\mathbb{C}\left[\mathfrak{X}_{\mathfrak{p}}^{e}\right] \cong \mathbb{C}\left[\mathcal{S}_{e} \cap\left(G \cdot \mathfrak{p}^{\perp}\right)\right]$.

In the case when the map is injective, we can therefore think of $W(e, \mathfrak{p})_{\gamma}$ as a quantization of $\mathcal{S}_{e} \cap\left(G \cdot \mathfrak{p}^{\perp}\right)$. In particular, in type A, if $\mathfrak{p}$ corresponds to $\tau$ as in Section 3.3 .2 , then $W(e, \mathfrak{p})_{\gamma}$ quantizes the intersection $\mathcal{S}_{e} \cap \overline{\mathbb{O}_{\tau}}$, where $\mathbb{O}_{\tau} \subset \mathfrak{g l}_{N}$ is the nilpotent orbit of type $\tau$.

As discussed in Los12, Remark 5.2.2], the issue about the map $T^{*} X \rightarrow \mathfrak{g}^{*}$ also manifests in the natural map $W(e)_{\gamma} \rightarrow W(e, \mathfrak{p})_{\gamma}$ failing to be surjective on the associated graded for the most obvious filtrations on these algebras.

Let $\left(\tilde{\mathscr{D}}_{\gamma}, \mathfrak{m}_{\chi}\right)$-mod denote the category of sheaves of $\tilde{\mathscr{D}}_{\gamma}$-modules on which the module action of $\mathfrak{m}_{\chi}$ integrates to a group action of the unipotent group $M$. Let 
$\tilde{\mathscr{Q}}_{\pi}=\tilde{\mathscr{D}}_{\gamma} / \tilde{\mathscr{D}}_{\gamma} \mathfrak{m}_{\chi}$. By Theorem 3.11 we have that $\tilde{\mathscr{D}}_{\gamma} \otimes_{\tilde{A}_{\gamma}}-$ is left and right adjoint to the sections functor $\Gamma$. Thus, we have that

$$
W \Gamma(\mathcal{M})=\operatorname{Hom}\left(\tilde{\mathscr{Q}}_{\pi}, \mathcal{M}\right)=\operatorname{Hom}\left(\tilde{Q}_{\pi}, \Gamma(M)\right),
$$

where $\tilde{Q}_{\pi}=\tilde{A}(\mathfrak{p})_{\gamma} / \tilde{A}(\mathfrak{p})_{\gamma} \mathfrak{m}_{\chi}$. Combining this with [Web11, Proposition 10], we obtain the result:

Corollary 3.21. The functor $W \Gamma=\operatorname{Hom}\left(\tilde{\mathscr{Q}}_{\pi},-\right):\left(\tilde{\mathscr{D}}_{\gamma}, \mathfrak{m}_{\chi}\right)-\bmod \rightarrow \tilde{A}(e, \mathfrak{p})_{\gamma}$-mod is an equivalence of categories.

3.3.4. Aside: $B$-algebras. Given a $\mathbb{Z}$-graded algebra $A$, the $B$-algebra with respect to this grading is the quotient $B(A)=A^{0} / \sum_{k \in \mathbb{Z}>0} A^{-k} A^{k}$ [BLPW16, Section 5.1]. As we'll now recall, this algebra controls aspects of the highest weight theory for $A$. In the context of symplectic duality it also has geometric significance, for example, as a cohomology ring by Hikita's conjecture [Hik17] and its extension by Nakajima; see KTWWY19a, Sections 1.6 and 8].

In particular, if $M$ is an $A$-module and $m \in M$ a "highest-weight" element (i.e., $A^{0} m \subseteq \mathbb{C} m$ and $A^{k} m=0$ for $k>0$ ), then there is an induced action of $B(A)$ on the line $\mathbb{C} m$. Conversely, for any homomorphism $B(A) \rightarrow \mathbb{C}$ we get a composed homomorphism

$$
A^{\geq 0}=\bigoplus_{k \geq 0} A^{k} \rightarrow B(A) \rightarrow \mathbb{C}
$$

and so an induced $A$-module $A \otimes_{A \geq 0} \mathbb{C}$ called a standard module. The element $m \in 1 \otimes 1$ is highest weight; this construction is left adjoint to that described above.

Remark 3.22. Suppose $S \subset A^{0}$ is a subalgebra which is central. Then for any commutative $S$-algebra $S^{\prime}$, we can extend the grading to $A \otimes_{S} S^{\prime}$. There is then a natural isomorphism $B\left(A \otimes_{S} S^{\prime}\right) \cong B(A) \otimes_{S} S^{\prime}$.

If $A$ is a commutative ring, then its $\mathbb{Z}$-grading corresponds to a $\mathbb{G}_{m}$-action on Spec $A$. In this case, $B(A)$ is canonically isomorphic to the coordinate ring of the scheme-theoretic fixed-point locus $(\operatorname{Spec} A)^{\mathbb{G}_{m}}$. We can leverage this in the noncommutative situation: when $A$ is the global section ring of a quantized conical symplectic resolution $\mathfrak{M}$, there is an inequality

$$
\operatorname{dim}_{\mathbb{C}} \mathbb{C}\left[\mathfrak{M}^{\mathbb{G}_{m}}\right]=\operatorname{dim}_{\mathbb{C}} B(\mathbb{C}[\mathfrak{M}]) \geq \operatorname{dim}_{\mathbb{C}} B(A),
$$

by [BLPW16, Proposition 5.1].

3.3.5. Highest weights in type A. We now return to the type A setting and set $\mathfrak{g}=\mathfrak{g l}_{N}$. Fix partitions $\pi, \tau \vdash N$. We let $e_{\pi} \in \mathfrak{g}$ be the nilpotent defined in Section 3.1.1, and $\mathfrak{p} \subset \mathfrak{g}$ (resp., $P \subset \mathrm{GL}_{N}$ ) be the parabolic subalgebra (resp., subgroup) corresponding to $\tau$. We fix also a character $\gamma: \mathfrak{p} \rightarrow \mathbb{C}$ and the corresponding set of parameters $\mathbf{R}$ as defined in Section 3.3.2. We set $W(\pi, \mathfrak{p})=W\left(e_{\pi}, \mathfrak{p}\right), A(\pi, \mathfrak{p})=$ $A\left(e_{\pi}, \mathfrak{p}\right), W(\pi, \mathfrak{p})_{\mathbf{R}}=W\left(e_{\pi}, \mathfrak{p}\right)_{\mathbf{R}}$, etc. Assume $w \in W=S_{N}$ is simultaneously a longest left coset representative of $W_{\pi}$ and a shortest right coset representative for $W_{\tau}$. We call such a permutation parabolic-singular and let $\mathcal{P S}(\pi, \mathfrak{p})$ be the set of such permutations. This set is in bijection with the set of row-strict tableaux of shape $\tau$ and type $\pi$, sending a tableau to the unique longest permutation that sends its row reading word to a weakly ordered one. Then the transposed version of [BO11, Corollary 2.6] shows that $\mathcal{P S}(\pi, \mathfrak{p})$ is non-empty if and only if $\tau \leq \pi^{t}$ in the dominance order. 
Let $\mathfrak{c}=\left\{h \in \mathfrak{h} \mid\left[h, e_{\pi}\right]=0\right\}$ be the centralizer of $e_{\pi}$ in the Cartan subalgebra $\mathfrak{h}$. If we complete $e_{\pi}$ to an $\mathfrak{s l}_{2}$ triple, this will also centralize $f_{\pi}$. In fact, the simultaneous centralizer of $e_{\pi}$ and $f_{\pi}$ is reductive with $\mathfrak{c}$ a Cartan.

The algebra $W(\pi, \mathfrak{p})$ carries an action of the group $C$ exponentiating $\mathfrak{c}$, and every cocharacter into this group induces a grading on $W(\pi, \mathfrak{p})$. We choose the grading induced by any dominant cocharacter which is generic in $\mathfrak{c}$ and consider the associated $B$-algebra $B(W(\pi, \mathfrak{p}))$. By [BLPW16, 5.1], this algebra is finite over $S=\operatorname{Sym}(\mathfrak{p} /[\mathfrak{p}, \mathfrak{p}])$.

We will be particularly interested in the related algebras $B\left(W(\pi, \mathfrak{p})_{\mathbf{R}}\right)$ and $B\left(\tilde{A}(\pi, \mathfrak{p})_{\gamma}\right)$; these are the base change of $B(A(\pi, \mathfrak{p}))$ to the closed point $\gamma$ and to the generic point of its formal neighborhood, respectively, as in Remark 3.22 . Thus, given a point $\nu \in \operatorname{Spec} B\left(\tilde{A}(\pi, \mathfrak{p})_{\gamma}\right)$, we can takes its Zariski closure in $\operatorname{Spec} B(A(\pi, \mathfrak{p}))$ and intersect that with $\operatorname{Spec} B\left(W(\pi, \mathfrak{p})_{\mathbf{R}}\right)$. Since $\operatorname{Spec} B(A(\pi, \mathfrak{p}))$ is finite and thus proper over $S$, this intersection will be a single point, which we call its specialization. For a general finite map, we could have points in Spec $B\left(W(\pi, \mathfrak{p})_{\mathbf{R}}\right)$ which are not the specialization of a more generic point, but this will not happen if $B(W(\pi, \mathfrak{p}))$ is free as a module over $S$ (or equivalently, flat over $S)$.

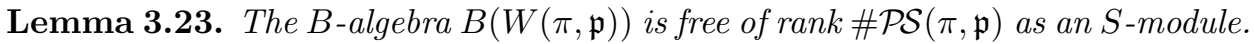
Thus, a weight for $W(\pi, \mathfrak{p})_{\mathbf{R}}$ is the highest weight of a module over $W(\pi, \mathfrak{p})_{\mathbf{R}}$ if and only if it is the specialization of the highest weight of a module over $\tilde{A}(\pi, \mathfrak{p})_{\gamma}$.

Proof. In order to show that an $S$-algebra is free of a given rank, it suffices to check that it has this rank generically and that there is no closed point where the rank of the base change is larger.

On the one hand, the base change of $B(A(\pi, \mathfrak{p}))$ to the generic point $B \otimes_{S} K$

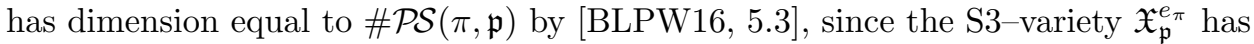
a torus action with fixed points in bijection with $\mathcal{P S}(\pi, \mathfrak{p})$.

On the other hand, by (3.13) the dimension of $B\left(W(\pi, \mathfrak{p})_{\mathbf{R}}\right)$ is bounded above by the "commutative $B$-algebra": the quotient of $\mathbb{C}[\mathfrak{X}]$ by the ideal generated by functions of non-zero weight. By [Hik17, A.1 and A.2], this has dimension equal to the Euler characteristic of another S3 variety, taking the Slodowy slice to a regular element in the Levi $\mathfrak{l}$ of $\mathfrak{p}$ (which thus has Jordan type $\tau$ corresponding to the diagonal blocks of $\mathfrak{l}$ ), and $G / Q$, where $Q$ is a parabolic with $e$ regular in its Levi (so of type $\pi$ ). Thus $\mathfrak{p}$ and $\pi$ essentially switch roles. We can obtain a bijection between $\mathcal{P S}(\pi, \mathfrak{p})$ and $\mathcal{P S}(\tau, \mathfrak{q})$ by taking the inverse and multiplying by $w_{0}$. Note that this requires reversing the order on blocks of $\tau$, but this order is immaterial, so this presents no issue. Note the appearance of the same reversal in [BLPW16, 10.4-5]. Thus $B\left(W(\pi, \mathfrak{p})_{\mathbf{R}}\right) \leq \# \mathcal{P S}(\pi, \mathfrak{p})$.

Since the dimension of the fiber is a lower semi-continuous function, this shows that this dimension must be constant, and by the usual argument, $B(A(\pi, \mathfrak{p}))$ must be a free $S$-algebra.

Thus, we can use localization to find the highest weights of modules over $\tilde{A}(\pi, \mathfrak{p})_{\gamma}$, and thus over $W(\pi, \mathfrak{p})_{\mathbf{R}}$. Note that the parabolic-singular permutations are precisely the shortest right coset representatives such that $M$ acts freely on the Schubert cell $U w P / P$, where $U$ is the unipotent of a Borel subgroup containing $M$. We lose no generality by assuming we have conjugated so that this is the standard Borel $B$. These are precisely the Schubert cells that carry an $(M, \chi)$-equivariant 
local system $\mathscr{L}_{w}$ Web11, Definition 12]. That is, $\mathscr{L}_{w}$ is a coherent $D$-module, such that the action of $\mathfrak{m}$ on $\mathscr{L}_{w}$ by $m \cdot x=\alpha_{m} x-\chi(m) x$ integrates to an $M$ action (where $\alpha_{m}$ is the vector field on $X$ given by the infinitesimal action of $m$ ); cf. equation (3.11). For such $w$, we consider the $D$-modules on $X$ given by $\delta_{w}=i_{!} \mathscr{L}_{w}, \nabla_{w}=i_{*} \mathscr{L}_{w}$, where $i: U w P / P \rightarrow G / P$ is the natural inclusion.

We thus have a natural map $U(\mathfrak{c}) \rightarrow W(\pi)$, which is a quantum moment map for the induced action of $C$ on the Slodowy slice $\mathcal{S}_{\pi}$.

Lemma 3.24. The highest weight of $W \Gamma\left(\nabla_{w}\right)$ over the torus $\mathfrak{c}$ is given by

$$
w\left(\gamma+\rho_{P}\right)+\rho=w w_{0}\left(\gamma-\rho_{P}\right)+\rho .
$$

Proof. This is analogous to HTT08, 12.3.1]. Let $w \in \mathcal{P S}(\pi, \mathfrak{p})$ be a parabolicsingular permutation. The module $\nabla_{w}$ is a pushforward $\iota: w w_{0} U w_{0} P / P \hookrightarrow X$, and thus we can just compute the pushforward on this open subvariety. We can identify $w U_{-} P / P \cong \operatorname{Ad}_{w}\left(\mathfrak{u}_{-}\right)$, with the subvariety $U w P / P$ sent to $\mathfrak{u} \cap \operatorname{Ad}_{w}\left(\mathfrak{u}_{-}\right)=$ $\mathfrak{u} \cap \operatorname{Ad}_{w}\left(\mathfrak{u}_{-}\right)$since $w$ is a shortest right coset representative. Now we enumerate the roots in $\operatorname{Ad}_{w}\left(\mathfrak{u}_{-}\right)$by $\beta_{1}, \ldots, \beta_{N}$, with the first $k$ roots $\left\{\beta_{1}, \ldots, \beta_{k}\right\}$ being those that are positive. Let $x_{i}$ denote the corresponding coordinates on $\operatorname{Ad}_{w}\left(\mathfrak{u}_{-}\right)$and $y_{i}$ the dual basis of $\operatorname{Ad}_{w}\left(\mathfrak{u}_{-}\right)$. We can also assume that $\left\{\beta_{1}, \ldots, \beta_{p}\right\}$ for some $p \leq k$ are the (necessarily simple) weight spaces on which $\chi$ is non-zero. The fact that $w$ is a longest left coset representative guarantees that any such weight space lies in $\operatorname{Ad}_{w}\left(\mathfrak{u}_{-}\right)$, so the parabolic-singular property shows that these lie in $\operatorname{Ad}_{w}\left(\mathfrak{u}_{-}\right)$.

Thus, we can identify the pushforward of $\mathscr{L}_{w}$ to this affine space with the module over the Weyl algebra $\mathbb{W}=\mathbb{C}\left[\mathfrak{u}_{-}\right] \otimes \operatorname{Sym}\left(\mathfrak{u}_{-}\right)$of $\mathfrak{u}_{-}$which is generated by a single element $e^{\chi}$ with the relations $\frac{\partial}{\partial x_{i}} e^{\chi}=\chi\left(y_{i}\right) e^{\chi}$ for $i=1, \ldots, k$, and $x_{i} \cdot e^{\chi}=0$ for $i=k+1, \ldots, n$. The function $e^{\chi}$ generates the Whittaker functions under multiplication by functions which are constant on $M$-orbits and multiplication by constant vector fields. In these coordinates, we have that $\mathfrak{c}$ acts by the Euler operator

$$
h \mapsto w\left(\gamma+\rho-\rho_{P}\right)(h)+\sum_{i=1}^{N} \beta_{i}(h) x_{i} \frac{\partial}{\partial x_{i}} .
$$

Note that since $\mathfrak{c}$ commutes with $e$, we have that $\beta_{i}(h)=0$ if $i \leq p$. On the function $e^{\chi}$, we have that

$$
x_{i} \frac{\partial}{\partial x_{i}} e^{\chi}= \begin{cases}\chi\left(y_{i}\right) x_{i} e^{\chi} & i \leq p, \\ 0 & p<i \leq k, \\ -\beta_{i}(h) & k<i .\end{cases}
$$

Thus, equation (3.14) becomes

$$
h \cdot e^{\chi} \mapsto\left(w\left(\gamma+\rho-\rho_{P}\right)(h)+\sum_{i=k+1}^{n} \beta_{i}(h)\right) e^{\chi} .
$$

Note here that $\beta_{i}$ ranges over the roots in $\operatorname{Ad}_{w}\left(\mathfrak{u}_{-}\right) \cap \mathfrak{u}_{-}=\operatorname{Ad}_{w w_{0}^{P}}\left(\mathfrak{u}_{-}\right) \cap \mathfrak{u}_{-}$, so the sum is $\rho-w w_{0}^{P} \rho=\rho-w \rho+2 w \rho_{P}$. Thus, we have that the weight of $e^{\chi}$ is $w\left(\gamma+\rho_{P}\right)+\rho=w w_{0}\left(\gamma-\rho_{P}\right)+\rho$.

We call $\gamma \in \mathfrak{h}^{*} \otimes_{\mathbb{C}} S$ row-sum-distinct if the restrictions $\left.w \cdot\left(\gamma+\rho_{P}\right)\right|_{\mathfrak{c}}$ are distinct for $w \in \mathcal{P S}(\pi, \mathfrak{p})$. Note that this is stronger than having stabilizer $W_{P}$, as the case of $N=4, \pi=(2,2), \mathfrak{p}=\mathfrak{b}$, and $\gamma=(4,3,2,1)$ shows: the permutations 
$(2,4,1,3)$ and $(2,4,3,1)$ are both parabolic-singular, but $w \cdot\left(\gamma+\rho_{P}\right)$ have the same restriction to $\mathfrak{c}=\{\operatorname{diag}(a, a, b, b): a, b \in \mathbb{C}\}$. For a fixed $P$, this is an open condition determined by finitely many hyperplanes.

In particular, the weight $\gamma+\iota$ for $\gamma \in \mathfrak{h}^{*}$ is always row-sum-distinct, since if $\left.w \cdot\left(\gamma+\iota+\rho_{P}\right)\right|_{\mathfrak{c}}=\left.w^{\prime} \cdot\left(\gamma+\iota+\rho_{P}\right)\right|_{\mathfrak{c}}$ for $w \neq w^{\prime} \in \mathcal{P S}(\pi, \mathfrak{p})$, then we must have $\left.w \cdot\left(\gamma+\rho_{P}\right)\right|_{\mathfrak{c}}=\left.w^{\prime} \cdot\left(\gamma+\rho_{P}\right)\right|_{\mathfrak{c}}$ and thus $\left.w \cdot \iota\right|_{\mathfrak{c}}=\left.w^{\prime} \cdot \iota\right|_{\mathfrak{c}}$. In this case, $w$ and $w^{\prime}$ are in the same double coset and thus must be equal (since each double coset contains at most one parabolic-singular permutation).

This actually shows something stronger: the difference $\left.w \cdot\left(\gamma+\iota+\rho_{P}\right)\right|_{\mathfrak{c}}-w^{\prime}$. $\left.\left(\gamma+\iota+\rho_{P}\right)\right|_{\mathfrak{c}}$ is never an integral weight (since its values are never scalars).

Recall that we have fixed a character $\gamma: \mathfrak{p} \rightarrow \mathbb{C}$ and the corresponding set of parameters $\mathbf{R}$ as defined in Section 3.3.2. For a given $w \in \mathcal{P S}(\pi, \mathfrak{p})$, we consider the weight $w \cdot\left(\gamma+\rho_{P}\right)$. We let

$$
\mathrm{T}_{w} \in \operatorname{Row}_{\widetilde{\mathbf{R}}}(\pi)
$$

be the row-symmetrized tableau of shape $\pi$ which has $w \cdot\left(\gamma+\rho_{P}\right)$ as a row reading (this corresponds to a filling in the alphabet $\widetilde{\mathbf{R}}$ ). We let $L_{w}$ be the simple module attached to this tableau by Brundan and Kleshchev (cf. Section 3.2.2). We let $\widetilde{\mathbf{T}}_{w}$ and $\widetilde{L}_{w}, \widetilde{\nabla}_{w}$ be corresponding objects for $\gamma+\iota$, base changed to $K$.

Lemma 3.25. We have an isomorphism $\widetilde{L}_{w} \cong W \Gamma\left(\widetilde{\nabla}_{w}\right)$.

Proof. By row-sum-distinctness, each of the simple modules has distinct highest weights for $\mathfrak{c}$, as do $W \Gamma\left(\tilde{\nabla}_{w}\right)$. In fact, since the weights of different $W \Gamma\left(\tilde{\nabla}_{w}\right)$ 's are never congruent modulo integral weights, there are no $\mathfrak{c}$-equivariant maps between them, and thus no $W(\pi, \mathfrak{p})$-equivariant ones. Thus, $W \Gamma\left(\tilde{\nabla}_{w}\right)$ will be simple and isomorphic to whichever of the modules $\tilde{L}_{w^{\prime}}$ has the same highest weight for $\mathfrak{c}$. By construction, this is $\tilde{L}_{w}$.

Theorem 3.26. The highest weights of modules in category $\mathcal{O}$ over $W(\pi, \mathfrak{p})_{\mathbf{R}}$ are given by the tableaux $\mathrm{T}_{w}$ for $w \in \mathcal{P S}(\pi, \mathfrak{p})$.

Proof. First we check this for $\gamma+\iota$ after base change to $K$. In this case, the simple modules are given by $\tilde{L}_{w}$. By Lemma 3.23 , the simples at $\gamma$ have highest weights obtained by specialization; that is, they are $L_{w}$.

Lemma 3.27. The action of $W(\pi, \mathfrak{p})_{\mathbf{R}}$ on $W \Gamma\left(\nabla_{w}\right)$ is faithful.

Proof. The module $\nabla_{w}$ is a naive pushforward from the open subset $w w_{0} U w_{0} P / P \subset$ $X$, so we can show faithfulness on this open subset. On this open subset, $\nabla_{w}$ is the pushforward of the Whittaker functions on an affine subspace, which is faithful.

A standard argument shows that a faithful module of finite length over a domain must have a faithful composition factor. Equivalently, we have that $J_{\mathfrak{p}}$ is the intersection of the annihilators of $W(\pi)_{\widetilde{\mathbf{R}}}$ acting on the composition factors of $W \Gamma\left(\nabla_{w}\right)$. Thus, we have:

Theorem 3.28. The algebra $W(\pi, \mathfrak{p})_{\mathbf{R}}$ acts faithfully on at least one $L_{w}$ for $w \in$ $\mathcal{P S}(\pi, \mathfrak{p})$; that is,

$$
W(\pi, \mathfrak{p})_{\mathbf{R}} \cong W(\pi)_{\widetilde{\mathbf{R}}} / \bigcap_{w \in \mathcal{P S}(\pi, \mathfrak{p})} \operatorname{Ann}\left(L_{w}\right) .
$$




\section{The quantized Mirković-Vybornov isomorphism}

Throughout this section we let $\mathfrak{g}=\mathfrak{s l}_{n}$.

4.1. The main theorem. Recall our notation from $1.2, \lambda \geq \mu$ are dominant coweights of $\mathfrak{g}$, and $\tau \geq \pi$ are partitions of $N$. Let $\mathfrak{p} \subset \mathfrak{g l}_{N}$ be the parabolic subalgebra corresponding to $\tau$.

Recall from (2.2) that for $\Lambda \in \operatorname{Gr}_{\mu}^{\bar{\lambda}}$, we have $\Lambda \subset \Lambda_{0}$ with $\Lambda_{0} / \Lambda \cong \mathbb{C}^{N}$ via the basis $E_{\pi}$. Furthermore the operator on $\Lambda_{0} / \Lambda$ induced by multiplication by $t$ is nilpotent of type $\leq \tau$. Therefore we have a map $\mathrm{Gr}_{\mu}^{\bar{\lambda}} \rightarrow \overline{\mathbb{O}_{\tau}} \subset \mathfrak{g l}_{N}$.

Theorem 4.1 (MV07b]). The map sending $\Lambda \in \mathrm{Gr}_{\mu}^{\bar{\lambda}}$ to the action of $t$ on $\Lambda_{0} / \Lambda \cong$ $\mathbb{C}^{N}$ defines an isomorphism of varieties

$$
\mathrm{Gr}_{\mu}^{\bar{\lambda}} \stackrel{\sim}{\longrightarrow} \mathcal{T}_{\pi} \cap \overline{\mathbb{O}_{\tau}}
$$

for a suitable transverse slice $\mathcal{T}_{\pi}$ to $\mathbb{O}_{\pi} \subset \overline{\mathbb{O}_{\tau}}$. Moreover, the following diagram commutes:

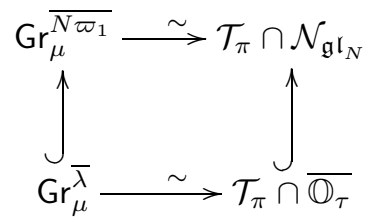

where the vertical arrows are the inclusions of closed subvarieties.

We review this theorem in more detail in Section 5.3 below.

Remark 4.2. In this paper, we use a formulation of the above result due to CautisKamnitzer [CK08, Section 3.3]. It is somewhat simpler than the original construction of Mirković-Vybornov. It is possible to modify the results of this paper to precisely match the original Mirković-Vybornov isomorphism; however, this comes at the cost of less pleasant maps of algebras and associated combinatorics.

We note that although in general $\mathcal{T}_{\pi}$ differs from the better-known Slodowy slice, these are isomorphic as Poisson varieties (cf. Section 5.1). Therefore the above theorem implies that $\mathcal{S}_{\pi} \cap \overline{\mathbb{O}_{\tau}} \cong \mathrm{Gr}_{\mu}^{\bar{\lambda}}$, where $\mathcal{S}_{\pi}$ is the Slodowy slice.

Now, on the one hand $\mathrm{Gr}_{\mu}^{\bar{\lambda}}$ is quantized by $Y_{\mu}^{\lambda}(\mathbf{R})$ for any set of parameters $\mathbf{R}$. On the other hand, $\mathcal{S}_{\pi} \cap \overline{\mathbb{O}_{\tau}}$ is quantized by $W(\pi, \mathfrak{p})_{\mathbf{R}}$. Our main result shows that we can lift the isomorphism of Mirković and Vybornov to the level of quantizations.

\section{Theorem 4.3.}

(a) There is an isomorphism of filtered algebras

$$
\Phi: Y_{\mu}^{N \varpi_{1}} \stackrel{\sim}{\longrightarrow} W(\pi)
$$

compatible with specialization of parameters on both sides. It identifies the Gelfand-Tsetlin subalgebras $\Gamma_{\mu}^{N \varpi_{1}} \stackrel{\sim}{\longrightarrow} \Gamma(\pi)$. 
(b) $\Phi$ induces a bijection between highest weight modules over $Y_{\mu}^{N \varpi_{1}}$ and $W(\pi)$, such that
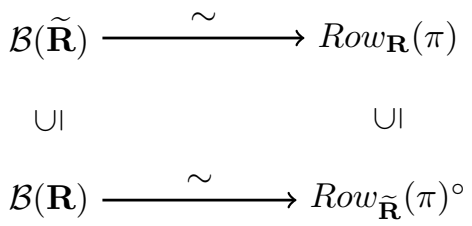

when $\widetilde{\mathbf{R}}$ and $\mathbf{R}$ are related as in (2.15). (Here $\operatorname{Row}_{\widetilde{\mathbf{R}}}(\pi)^{\circ}$ is a set of row symmetrized $\pi$-tableaux parametrizing highest weights for $W(\pi, \mathfrak{p})_{\mathbf{R}}$; see Section 4.3.2.)

(c) $\Phi$ induces an isomorphism of filtered algebras

$$
Y_{\mu}^{\lambda} \stackrel{\sim}{\longrightarrow} W(\pi, \mathfrak{p}) .
$$

It is compatible with specializations of parameters on both sides, yielding isomorphisms $Y_{\mu}^{\lambda}(\mathbf{R}) \stackrel{\sim}{\longrightarrow} W(\pi, \mathfrak{p})_{\mathbf{R}}$ for any set of parameters $\mathbf{R}$ via the commutative diagram

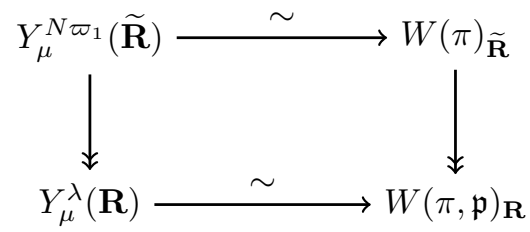

These isomorphisms identify the Gelfand-Tsetlin subalgebras $\Gamma_{\mu}^{\lambda} \stackrel{\sim}{\longrightarrow} \Gamma(\pi, \mathfrak{p})$ and $\Gamma_{\mu}^{\lambda}(\mathbf{R}) \stackrel{\sim}{\longrightarrow} \Gamma(\pi, \mathfrak{p})_{\mathbf{R}}$.

(d) The classical limit agrees with the $M V$ isomorphism.

We will split the proof of this theorem into parts, which occupy the remainder of the paper. In Sections 4.2 4.3, and 5, we will prove parts (a), (b), and (d) of this theorem, respectively. Part (c) follows from parts (a), (b) by a simple argument, as we will show presently.

This linkage uses the quotient maps

$$
Y_{\mu}^{N \varpi_{1}} \rightarrow Y_{\mu}^{\lambda}, \quad W(\pi) \rightarrow W(\pi, \mathfrak{p}) .
$$

introduced in Sections 2.6 and 3.3.3. respectively. Note that these maps are not surjective; instead it is better to work with the surjective maps

$$
Y_{\mu}^{N \varpi_{1}}(\tilde{\gamma}) \rightarrow Y_{\mu}^{\lambda}(\gamma), \quad A(\pi, \mathfrak{b}) \rightarrow A(\pi, \mathfrak{p})
$$

The subalgebras $Y_{\mu}^{\lambda} \subset Y_{\mu}^{\lambda}(\gamma)$ generate $Y_{\mu}^{\lambda}(\gamma)$ over its center, and similarly for $W(\pi, \mathfrak{b}) \subset A(\pi, \mathfrak{b})$.

From part (a), we can construct an $S_{N}$-equivariant isomorphism $Y_{\mu}^{N \varpi_{1}}(\tilde{\gamma}) \cong$ $A(\pi, \mathfrak{b})$ by base change. If we show that we have an induced isomorphism $Y_{\mu}^{\lambda}(\gamma) \cong$ $A(\pi, \mathfrak{p})$, this will necessarily be equivariant for the actions of $\Theta$ on $Y_{\mu}^{\lambda}(\gamma)$ by permuting formal roots as in Remark 2.3 and on $A(\pi, \mathfrak{p})$ by the action discussed in Section 3.3.1. We thus obtain the desired map in Theorem 4.3. (c) by taking invariants of these $\Theta$-actions. 
Proof of Theorem 4.3(c) (assuming parts (a) and (b)). In order to complete the proof, we need only show that the kernels in (4.1) match under $\Phi$ after base change at these maximal ideals. By Theorem 3.28, the kernel of the latter map is the intersection of the annihilators of the simple modules over $Y_{\mu}^{\lambda}(\gamma)$. By Theorem 4.3(b) and Corollary 2.16, we thus obtain an induced surjective map $Y_{\mu}^{\lambda}(\gamma) \rightarrow A(\pi, \mathfrak{p})$.

Recall that by Corollary 2.8, $Y_{\mu}^{\lambda}(\gamma)$ is a free module of infinite rank over its center. Thus, if this map is not injective, it will fail to be injective after quotient by any maximal ideal of the center. Similarly, since each piece of the filtration of $Y_{\mu}^{\lambda}(\gamma)$ and $A(\pi, \mathfrak{p})$ is finite dimensional, if the map $\Phi$ is not an isomorphism of filtered algebras, it will fail to be an isomorphism after specialization at a maximal ideal in the center. Thus, we can consider the quotient $Y_{\mu}^{\lambda}(\mathbf{R})$ with $\mathbf{R}$ giving a maximal ideal of the center and the corresponding quotient of $W(\pi, \mathfrak{p})_{\mathbf{R}}$.

When we take associated graded of both sides, we obtain the functions on $\mathrm{Gr}_{\mu}^{\bar{\lambda}}$ (Theorem 2.5) and $\mathcal{S}_{\pi} \cap \overline{\mathbb{O}_{\tau}}$, respectively. Both are irreducible varieties of the same dimension; thus a surjective ring map from one to the other must be an isomorphism. Since $\Gamma(\pi, \mathfrak{p})$ is the image of $\Gamma(\pi)$ by Definition 3.19 and $\Gamma_{\mu}^{\lambda}$ the image of $\Gamma_{\mu}^{N \varpi_{1}}$ by Theorem 2.13, the compatibility of Gelfand-Tsetlin subalgebras follows from the same statement for the case $\lambda=N \varpi_{1}$.

We next note an immediate corollary about the original Mirković-Vybornov isomorphism:

Corollary 4.4. $\operatorname{Gr}_{\mu}^{\bar{\lambda}}$ and $\mathcal{T}_{\pi} \cap \overline{\mathbb{O}_{\tau}}$ are isomorphic as Poisson varieties. This isomorphism intertwines the $\mathbb{C}^{\times}$-action by loop rotation on $\mathrm{Gr}_{\mu}^{\bar{\lambda}}$ with the square root

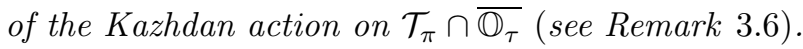

Remark 4.5. In the classical limit, the Gelfand-Tsetlin subalgebras $\Gamma_{\mu}^{\lambda}(\mathbf{R}) \subset Y_{\mu}^{\lambda}(\mathbf{R})$ and $\Gamma(\pi, \mathfrak{p})_{\mathbf{R}} \subset W(\pi, \mathfrak{p})_{\mathbf{R}}$ become Poisson commutative subalgebras of the coordinate rings of $\mathrm{Gr}_{\mu}^{\bar{\lambda}}$ and $\mathcal{T}_{\pi} \cap \overline{\mathbb{O}_{\tau}}$, respectively. In other words, they define integrable systems. It follows that the Mirković-Vybornov isomorphism also intertwines these integrable systems.

We note a second immediate corollary, which establishes a generalization of [FMO10, Conjecture 2] for all parabolic W-algebras. By combining part (c) of the main theorem with Corollary 2.8, we deduce:

Corollary 4.6. $W(\pi, \mathfrak{p})$ is free as a left (or right) module over its Gelfand-Tsetlin subalgebra $\Gamma(\pi, \mathfrak{p})$.

4.2. Proof of Theorem 4.3(a): The case of $\lambda=N \varpi_{1}$. In this section, we will consider the case where $\lambda=N \varpi_{1}$ and $\mu$ is a dominant weight such that $\mu \leq \lambda$. From this data, we have a partition $\pi \vdash N$ as in Section 1.2. We'll describe an

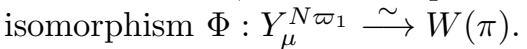

To state the theorem precisely, first we need to define a map

$$
\phi: Y_{\mu} \rightarrow S Y_{n}(\sigma)
$$


by

$$
\begin{aligned}
H_{i}(u) & \mapsto \frac{\left(u+\frac{i-1}{2}\right)^{\mu_{i}}}{u^{\mu_{i}}} \frac{D_{i+1}\left(-u-\frac{i-1}{2}\right)}{D_{i}\left(-u-\frac{i-1}{2}\right)}, \\
E_{i}(u) & \mapsto E_{i}\left(-u-\frac{i-1}{2}\right), \\
F_{\mu, i}(u) & \mapsto(-1)^{\mu_{i}} F_{\mu, i}\left(-u-\frac{i-1}{2}\right)
\end{aligned}
$$

for $i \in I$. Here $F_{\mu, i}(u)=\sum_{r>0} F_{i}^{\left(\mu_{i}+r\right)} u^{-r}$ on each side.

Proposition 4.7. The map $\phi$ is an isomorphism of filtered algebras.

For the proof, we will make use of the following lemma regarding "non-standard" embeddings of the shifted Yangian $Y_{\mu} \hookrightarrow Y$ :

Lemma 4.8. Fix a monic polynomial

$$
P_{i}(u)=u^{\mu_{i}}+P_{i}^{(1)} u^{\mu_{i}-1}+\cdots+P_{i}^{\left(\mu_{i}\right)} \in \mathbb{C}[u]
$$

for each $i=1, \ldots, n-1$. There is a corresponding embedding $Y_{\mu} \hookrightarrow Y$, defined on the generators by

$$
\begin{aligned}
E_{i}^{(r)} & \mapsto E_{i}^{(r)} \\
H_{i}^{(r)} & \mapsto H_{i}^{(r)}+P_{i}^{(1)} H_{i}^{(r-1)}+\cdots+P_{i}^{\left(\mu_{i}\right)} H_{i}^{\left(r-\mu_{i}\right)}, \\
F_{i}^{(s)} & \mapsto F_{i}^{(r)}+P_{i}^{(1)} F_{i}^{(s-1)}+\cdots+P_{i}^{\left(\mu_{i}\right)} F_{i}^{\left(s-\mu_{i}\right)}
\end{aligned}
$$

for all $r>0$ and $s>\mu_{i}$, and where we interpret $H_{i}^{(0)}=1$ and $H_{i}^{(r)}=0$ for $r<0$.

Proof. Assuming that this map defines a homomorphism, it is easy to see that it is an embedding: its associated graded agrees with that of the defining embedding $Y_{\mu} \subset Y$.

To prove that it is a homomorphism, one can verify the relations directly; we give a different argument. By [KTWWY19a, Lemma 3.7], $Y_{\mu}$ is a left coideal of $Y$ with respect to its defining embedding $Y_{\mu} \subset Y$ (see Definition 2.1). By [KTWWY19a, Proposition 3.8], there is a 1-dimensional module $\mathbb{C} \mathbf{1}_{Q}$ for $Y_{\mu}$ determined by the polynomials $P_{i}(u)$. We can then consider

$$
Y_{\mu} \stackrel{\Delta}{\longrightarrow} Y \otimes Y_{\mu} \longrightarrow Y \otimes \operatorname{End}\left(\mathbb{C} \mathbf{1}_{Q}\right) \cong Y .
$$

The composition is precisely the claimed homomorphism.

Proof of Proposition 4.7. When $\mu=0$ the fact that this map defines an isomorphism $Y \stackrel{\sim}{\rightarrow} S Y_{n}$ follows from [BK05, Remark 5.12] after a minor modification: here we are following Drinfeld's conventions as opposed to the "opposite" presentation of BK05].

When $\mu \neq 0$, consider the composition

$$
Y_{\mu} \hookrightarrow Y \stackrel{\sim}{\longrightarrow} S Y_{n},
$$

where the second arrow is the above $\mu=0$ isomorphism, while the first arrow is the embedding from the previous lemma for the polynomials $P_{i}(u)=\left(u+\frac{i-1}{2}\right)^{\mu_{i}}$. This map $Y_{\mu} \hookrightarrow S Y_{n}$ agrees with $\phi$ on the generators of $Y_{\mu}$, and its image is precisely $S Y_{n}(\sigma)$. 
Recall the algebra $Y_{\mu}\left[R^{(j)}\right]$ from (2.3); note that as $\lambda=N \varpi_{1}$ we only adjoin variables $R^{(j)}:=R_{n-1}^{(j)}$ for $j=1, \ldots, N$. We extend $\phi$ to an isomorphism $\phi$ : $Y_{\mu}\left[R^{(j)}\right] \rightarrow S Y_{n}(\sigma) \otimes \mathbb{C}\left[Z^{(1)}, \ldots, Z^{(N)}\right]$, where the $Z^{(j)}$ are formal variables. On the central generators $\phi$ is defined by the equation

$$
(-1)^{N} R\left(-u+\frac{n}{2}\right) \mapsto u^{N}+Z^{(1)} u^{N-1}+\cdots+Z^{(N)}=: Z_{N}(u) .
$$

We now consider the following diagram:

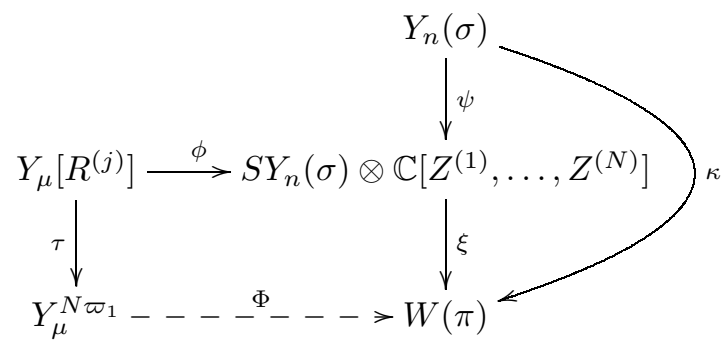

Here $\tau: Y_{\mu}\left[R^{(j)}\right] \rightarrow Y_{\mu}^{N \varpi_{1}}$ is the defining quotient map, while $\kappa: Y_{n}(\sigma) \rightarrow W(\pi)$ is Brundan and Kleshchev's surjection from Theorem 3.5. The map $\psi$ is the identity on $S Y_{n}(\sigma)$ and on the center is defined by the equation

$$
Z_{N}(u)=u^{p_{1}}(u-1)^{p_{2}} \cdots(u-n+1)^{p_{n}} \psi\left(Q_{n}(u)\right) .
$$

The map $\xi$ is equal to $\kappa$ on $S Y_{n}(\sigma)$ and on the center is defined by the equation

$$
\xi\left(Z_{N}(u)\right)=u^{p_{1}}(u-1)^{p_{2}} \cdots(u-n+1)^{p_{n}} \kappa\left(Q_{n}(u)\right) .
$$

Note that by [BK08, Lemma 3.7] the right-hand side of the above equation is a polynomial in $u$ of degree $N$, and hence $\xi$ is a well-defined surjection. By construction we have that $\kappa=\xi \circ \psi$.

We will now show that $\Phi$ exists by proving the equality $\phi\left(I_{\mu}^{\lambda}\right)=\operatorname{ker}(\xi)$. Notice that $\Phi$ is then an isomorphism. It also induces an isomorphism between the central quotients $Y_{\mu}^{N \varpi_{1}}(\mathbf{R}) \cong W(\pi)_{\mathbf{R}}$ because of (4.4) and the above discussion.

Our first order of business is to determine the image of $A_{i}^{(\ell)}$ under $\phi$. From the identity $D_{i}(u)=\frac{Q_{i}(u+i-1)}{Q_{i-1}(u+i-1)}$ of (3.5) we obtain that

$$
\frac{D_{i+1}\left(-u-\frac{i-1}{2}\right)}{D_{i}\left(-u-\frac{i-1}{2}\right)}=\frac{Q_{i-1}\left(-u+\frac{i-1}{2}\right)}{Q_{i}\left(-u+\frac{i-1}{2}\right)} \frac{Q_{i+1}\left(-u+\frac{i+1}{2}\right)}{Q_{i}\left(-u+\frac{i+1}{2}\right)}
$$

and hence the image

$$
\phi\left(H_{i}(u)\right)=\frac{\left(u+\frac{i-1}{2}\right)^{\mu_{i}}}{u^{\mu_{i}}} \frac{\psi\left(Q_{i-1}\left(-u+\frac{i-1}{2}\right)\right)}{\psi\left(Q_{i}\left(-u+\frac{i-1}{2}\right)\right)} \frac{\psi\left(Q_{i+1}\left(-u+\frac{i+1}{2}\right)\right)}{\psi\left(Q_{i}\left(-u+\frac{i+1}{2}\right)\right)} .
$$

The next result is analogous to Corollary 2.18.

Lemma 4.9. There exist unique series $s_{i}(u) \in \mathbb{C}\left[Z^{(1)}, \ldots, Z^{(N)}\right]\left[\left[u^{-1}\right]\right]$ with constant term 1 such that

$$
\phi\left(A_{i}(u)\right)=s_{i}(u) \psi\left(Q_{i}\left(-u+\frac{i-1}{2}\right)\right)
$$

for $i=1, \ldots, n-1$. These satisfy the equations

$$
\phi\left(r_{i}(u)\right) \frac{s_{i-1}\left(u-\frac{1}{2}\right) s_{i+1}\left(u-\frac{1}{2}\right)}{s_{i}(u) s_{i}(u-1)}=\frac{\left(u+\frac{i-1}{2}\right)^{\mu_{i}}}{u^{\mu_{i}}}
$$


for $i=1, \ldots, n-2$ and

$$
\phi\left(r_{n-1}(u)\right) \frac{s_{n-2}\left(u-\frac{1}{2}\right)}{s_{n-1}(u) s_{n-1}(u-1)}=\frac{\left(u+\frac{n-2}{2}\right)^{\mu_{n-1}}}{u^{\mu_{n-1}}} \psi\left(Q_{n}\left(-u+\frac{n}{2}\right)\right) .
$$

Moreover, these equations determine the $s_{i}(u)$ uniquely.

Proof. For each $i$, we have two factorizations for $\phi\left(H_{i}(u)\right)$ : one in terms of $\phi\left(r_{i}(u)\right)$ and the $\phi\left(A_{j}(u)\right)$ by (2.4), and one in terms of the $\psi\left(Q_{j}(-u)\right.$ ) by (4.6) (with appropriate shifts in $u$ in both cases). The claim now follows by applying the uniqueness of such factorizations [GKLO05, Lemma 2.1].

Note that this result implies the desired match of Gelfand-Tsetlin subalgebras.

Lemma 4.10. For $i=1, \ldots, n-1$,

$$
s_{i}(u)=\frac{\left(u-\frac{i-1}{2}\right)^{p_{1}}\left(u-\frac{i-3}{2}\right)^{p_{2}} \cdots\left(u+\frac{i-1}{2}\right)^{p_{i}}}{u^{m_{i}}} .
$$

Proof. Denote the right-hand side of (4.9) by $x_{i}(u)$. By the previous lemma, it suffices to show that the $x_{i}(u)$ satisfy the equations (4.7), (4.8).

For the case of equation (4.8), the left-hand side is

$$
\begin{gathered}
\phi\left(r_{n-1}(u)\right) \cdot \frac{x_{n-2}\left(u-\frac{1}{2}\right)}{x_{n-1}(u) x_{n-1}(u-1)} \\
=u^{-N} \phi(R(u)) \frac{\left(1-\frac{1}{2} u^{-1}\right)^{m_{n-2}}}{\left(1-u^{-1}\right)^{m_{n-1}}} \cdot \frac{u^{m_{n-1}}(u-1)^{m_{n-1}}}{\left(u-\frac{1}{2}\right)^{m_{n-2}}\left(u+\frac{n-2}{2}\right)^{p_{n-1}} \prod_{j=1}^{n-1}\left(u-\frac{n}{2}+j-1\right)^{p_{j}}}
\end{gathered}
$$

after cancelling common factors between $x_{n-2}\left(u-\frac{1}{2}\right)$ and $x_{n-1}(u)$. This reduces to

$$
\frac{\phi(R(u))}{u^{p_{n}-p_{n-1}}} \frac{1}{\left(u+\frac{n-2}{2}\right)^{p_{n-1}} \prod_{j=1}^{n-1}\left(u-\frac{n}{2}+j-1\right)^{p_{j}}} .
$$

Now consider the right-hand side of (4.8). Applying (4.5) and (4.4), we get

$$
\begin{aligned}
& \frac{\left(u+\frac{n-2}{2}\right)^{\mu_{n-1}}}{u^{\mu_{n-1}}} \phi\left(Q_{n}\left(-u+\frac{n}{2}\right)\right) \\
= & \frac{\left(u+\frac{n-2}{2}\right)^{p_{n}-p_{n-1}}}{u^{p_{n}-p_{n-1}}} \frac{Z_{N}\left(-u+\frac{n}{2}\right)}{\left(-u+\frac{n}{2}\right)^{p_{1}}\left(-u+\frac{n-2}{2}\right)^{p_{2}} \cdots\left(-u-\frac{n-2}{2}\right)^{p_{n}}} \\
= & \frac{\left(u+\frac{n-2}{2}\right)^{p_{n}-p_{n-1}}}{u^{p_{n}-p_{n-1}}} \frac{(-1)^{N} \phi(R(u))}{(-1)^{N}\left(u-\frac{n}{2}\right)^{p_{1}}\left(u-\frac{n-2}{2}\right)^{p_{2}} \cdots\left(u+\frac{n-2}{2}\right)^{p_{n}}}
\end{aligned}
$$

and we see that the right and left sides agree.

Verifying that the $x_{i}(u)$ satisfy equation (4.7) for $1 \leq i<n-1$ is analogous and is left as an exercise for the reader.

Lemma 4.11. $\phi\left(I_{\mu}^{\lambda}\right) \subset \operatorname{ker}(\xi)$.

Proof. Combining the two lemmas,

$$
\phi\left(A_{i}(u)\right)=\frac{\left(u-\frac{i-1}{2}\right)^{p_{1}}\left(u-\frac{i-3}{2}\right)^{p_{2}} \cdots\left(u+\frac{i-1}{2}\right)^{p_{i}}}{u^{m_{i}}} \psi\left(Q_{i}\left(-u+\frac{i-1}{2}\right)\right) .
$$


We will now apply the definition (3.4) of $Q_{i}(u)$. By Theorem 3.5 in BK08, we have that $\kappa\left(T_{\ell k}^{(r)}\right)=0$ for $r>p_{\ell}$. Therefore for any $\ell=1, \ldots, n$,

$$
\frac{\left(u-\frac{i-1}{2}+\ell-1\right)^{p_{\ell}}}{u^{p_{\ell}}} \kappa\left(T_{\ell k}\left(-u+\frac{i-1}{2}-\ell+1\right)\right)
$$

is a polynomial in $u^{-1}$ of degree $p_{\ell}$. Observe by (3.4) that

$$
\begin{aligned}
\xi \circ \psi\left(Q_{i}\right. & \left.\left(-u+\frac{i-1}{2}\right)\right) \\
= & \sum_{w \in S_{n-i}}(-1)^{w} \kappa\left(T_{i, w(i)}\left(-u+\frac{i-1}{2}-i+1\right)\right) \cdots \kappa\left(T_{1, w(1)}\left(-u+\frac{i-1}{2}\right)\right) .
\end{aligned}
$$

Since $p_{1}+\cdots+p_{n-i}=m_{i}$, it follows that $\xi \circ \phi\left(A_{i}(u)\right)$ is a polynomial in $u^{-1}$ of degree $m_{i}$. This proves the claim.

Lemma 4.12. $\phi\left(I_{\mu}^{\lambda}\right) \supset \operatorname{ker}(\xi)$.

Proof. By Lemmas 4.9 and 4.10, we have

$$
\phi\left(A_{1}(u)\right)=s_{1}(u) \psi\left(Q_{1}(-u)\right)=\psi\left(Q_{1}(-u)\right) .
$$

Noting that $D_{1}(u)=Q_{1}(u)$, it follows that $\psi\left(D_{1}^{(r)}\right)=(-1)^{r} \phi\left(A_{1}^{(r)}\right)$.

By [BK08, Section 3.4] $\operatorname{ker}(\kappa)=\left\langle D_{1}^{(r)}: r>p_{1}\right\rangle$, so

$$
\operatorname{ker}(\xi)=\psi(\operatorname{ker}(\kappa))=\left\langle\psi\left(D_{1}^{(r)}\right): r>p_{1}\right\rangle .
$$

Since $p_{1}=m_{1}$, the elements $\phi\left(A_{1}^{(r)}\right) \in \phi\left(I_{\mu}^{\lambda}\right)$ for $r>p_{1}$. So $\operatorname{ker}(\xi) \subset \phi\left(I_{\mu}^{\lambda}\right)$.

This completes the proof of Theorem 4.3(a).

4.3. Proof of Theorem 4.3(b): The product monomial crystal and row tableaux. Let $\mathbf{R}$ be a set of parameters of weight $\lambda$ and define $\widetilde{\mathbf{R}}$ to be the corresponding set of parameters of weight $N \varpi_{1}$, as in Theorem 2.13. We let $\gamma$ be a $W_{P}$-invariant weight such that the values of the weight on blocks of size $i$ are given by the elements of $R_{i}$ with multiplicity; while this is not unique, its orbit under the Weyl group is. Note that the elements of $\widetilde{\mathbf{R}}$ are just the entries of $\gamma+\rho_{P}$.

Note that the isomorphism $\Phi$ preserves the notion of highest weight vector and highest weight module: it sends $E$ 's to $E$ 's and $H$ 's to $D$ 's. In this section we describe how the highest weights of $Y_{\mu}^{\lambda}(\mathbf{R})$ and $Y_{\mu}^{N \varpi_{1}}(\widetilde{\mathbf{R}})$ (as described in Section 2.5) match up respectively with the highest weights of $W(\pi)_{\widetilde{\mathbf{R}}}$ and $W(\pi, \mathfrak{p})_{\mathbf{R}}$ (as described in [BK08] and Section 3.2). That is, we will describe the commutative diagram

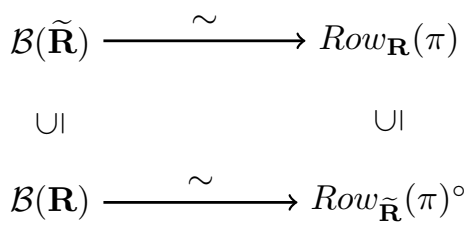

as prescribed by Theorem 4.3(b). Both vertical maps are natural inclusions of subsets, and the horizontal maps are bijections induced by $\Phi$. 
4.3.1. A bijection for $\lambda=N \varpi_{1}$. Consider the isomorphism $\Phi: Y_{\mu}^{N \varpi_{1}} \rightarrow W(\pi)$ from Section 4.2. By equation (4.4), it follows that $\Phi$ descends to an isomorphism

$$
Y_{\mu}^{N \varpi_{1}}(\widetilde{\mathbf{R}}) \cong W(\pi)_{\widetilde{\mathbf{R}}} .
$$

On the one hand, the set of highest weights $\mathcal{B}(\widetilde{\mathbf{R}})_{\mu}$ of $Y_{\mu}^{N \varpi_{1}}(\widetilde{\mathbf{R}})$ is in bijection with the set

$$
H_{\mu}^{N \varpi_{1}}(\widetilde{\mathbf{R}})=\left\{\left(\mathbf{S}_{i}\right)_{i \in I}: \begin{array}{l}
\left|\mathbf{S}_{i}\right|=m_{i} \text { and } \\
\mathbf{S}_{1}+n \subset \mathbf{S}_{2}+(n-1) \subset \cdots \subset \mathbf{S}_{n-1}+1 \subset \widetilde{\mathbf{R}}
\end{array}\right\} .
$$

As in (2.13), the highest weight corresponding to $\left(\mathbf{S}_{i}\right) \in H_{\mu}^{N \varpi_{1}}(\widetilde{\mathbf{R}})$ is given by

$$
A_{i}(u) \mapsto \prod_{s \in \mathbf{S}_{i}}\left(1-\frac{1}{2} s u^{-1}\right)=u^{-m_{i}} \prod_{s \in \mathbf{S}_{i}}\left(u-\frac{1}{2} s\right) .
$$

On the other hand, recall from Section 3.2 .2 that the set of highest weights for $W(\pi)_{\widetilde{\mathbf{R}}}$ is $\operatorname{Row}_{\widetilde{\mathbf{R}}}(\pi)$, the set of row symmetrized $\pi$-tableaux $T$ on the alphabet $\widetilde{\mathbf{R}}$, and that $T \in \operatorname{Row}_{\widetilde{\mathbf{R}}}(\pi)$ encodes a highest weight according to

$$
(u-i+1)^{p_{i}} D_{i}(u-i+1) \mapsto \prod_{a \in T_{i}}\left(u+\frac{1}{2} a-\frac{n}{2}\right) .
$$

Proposition 4.13. Let $\widetilde{\mathbf{R}}$ be a multiset of size $N$. The isomorphism $\Phi: Y_{\mu}^{N \varpi_{1}}(\widetilde{\mathbf{R}})$ $\rightarrow W(\pi)_{\widetilde{\mathbf{R}}}$ induces a bijection

$$
\operatorname{Row}_{\widetilde{\mathbf{R}}}(\pi) \rightarrow \mathcal{B}(\widetilde{\mathbf{R}})_{\mu}
$$

given by $T \mapsto \mathbf{S}=\left(\mathbf{S}_{i}\right)$, where

$$
\mathbf{S}_{i}=\left(T_{1} \cup \cdots \cup T_{i}\right)-(n-i+1),
$$

and $T_{i}$ denotes the $i$-th row of $T$.

Equivalently, the $i$-th row is obtained from

$$
T_{i}=\left(\mathbf{S}_{i}+(n-i+1)\right) \backslash\left(\mathbf{S}_{i-1}+(n-i+2)\right),
$$

that is, the difference between parts of the "flag" of multisets (4.11).

Proof. We begin with the equation

$$
(u-i+1)^{p_{i}} D_{i}(u-i+1)=(u-i+1)^{p_{i}} \frac{Q_{i}(u)}{Q_{i-1}(u)}=\frac{\left(u-\frac{i-1}{2}\right)^{m_{i}} \Phi\left(A_{i}\left(-u+\frac{i-1}{2}\right)\right)}{\left(u-\frac{i-1}{2}\right)^{m_{i-1}} \Phi\left(A_{i-1}\left(-u+\frac{i-2}{2}\right)\right)} .
$$

The first equality is equation (3.5), while the second equality follows from Lemmas 4.9, 4.10 after cancelling common factors.

For a highest weight $\mathbf{S}=\left(\mathbf{S}_{i}\right)$ for $Y_{\mu}^{N \varpi_{1}}(\widetilde{\mathbf{R}})$, the right-hand side maps to

$$
\frac{\prod_{s \in \mathbf{S}_{i}}\left(u+\frac{s-i+1}{2}\right)}{\prod_{s \in \mathbf{S}_{i-1}}\left(u+\frac{s-i+2}{2}\right)} \text {. }
$$

To find the corresponding tableaux $T \in \operatorname{Row}_{\widetilde{\mathbf{R}}}(\pi)$, we must write the above as

$$
\prod_{a \in T_{i}}\left(u+\frac{a}{2}-\frac{n}{2}\right)
$$

which leads to $T_{i}=\left(\mathbf{S}_{i}+(n-i+1)\right) \backslash\left(\mathbf{S}_{i-1}+(n-i+2)\right)$. This proves the proposition. 
4.3.2. A bijection for general $\lambda$. Next we'll prove that the bijection of Proposition 4.13 induces a bijection between the highest weights of $Y_{\mu}^{\lambda}(\mathbf{R})$ and the highest weights of $W(\pi, \mathfrak{p})_{\mathbf{R}}$. We'll do this by first identifying the tableaux in $\operatorname{Row}_{\widetilde{\mathbf{R}}}(\pi)$ which descend to highest weights of $W(\pi, \mathfrak{p})_{\mathbf{R}}$; we term these "overshadowing tableaux". Once this is done, we need only check that these satisfy the same conditions as the subcrystal $\mathcal{B}(\mathbf{R}) \subset \mathcal{B}(\widetilde{\mathbf{R}})$ (cf. Lemma 2.14). Let $\operatorname{Row}_{\widetilde{\mathbf{R}}}(\pi)^{\circ}$ denote the set of highest weights of $W(\pi, \mathfrak{p})_{\mathbf{R}}$. By Theorem 3.28 there is an inclusion $\operatorname{Row}_{\widetilde{\mathbf{R}}}(\pi)^{\circ} \subset \operatorname{Row}_{\widetilde{\mathbf{R}}}(\pi)$. Now suppose $c \in \mathbf{R}_{i}$. Then in $\widetilde{\mathbf{R}}$ the element $c$ has $n-i$ "descendants", namely, the elements

$$
\{c+n-i-1, c+n-i-3, \ldots, c-n+i+1\} .
$$

We'll call this set the $c$-block in $\widetilde{\mathbf{R}}$.

Given a row tableau $T \in \operatorname{Row}_{\widetilde{\mathbf{R}}}(\pi)$, we can divide the boxes of the tableau into $c$-blocks. Note that this decomposition will not be unique if $\widetilde{\mathbf{R}}$ contains any element with multiplicity greater than 1 . We say that the tableau $T$ is overshadowing if this division into $c$-blocks can be chosen so that for every $c \in \mathbf{R}$ the elements of the $c$-block occur in strictly decreasing order down the tableau.

Put another way, given $T \in \operatorname{Row}_{\widetilde{\mathbf{R}}}(\pi)$, an R-coloring of $T$ is a coloring of the contents of $T$ using $|\mathbf{R}|$ colors, such that for every $c \in \mathbf{R}$ the elements colored $c$ form a $c$-block, and they are in strictly decreasing order down the rows. Clearly $T$ is overshadowing if and only if there exists an $\mathbf{R}$-coloring of $T$.

Lemma 4.14. $\operatorname{Row}_{\widetilde{\mathbf{R}}}(\pi)^{\circ}$ is precisely the subset of overshadowing row tableaux in $\operatorname{Row}_{\widetilde{\mathbf{R}}}(\pi)$.

Proof. By Theorem 4.13, the set $\operatorname{Row}_{\widetilde{\mathbf{R}}}(\pi)^{\circ}$ is the set of tableaux where the row reading word is of the form $w \cdot\left(\gamma+\rho_{\mathfrak{p}}\right)$, for $w \in \mathcal{P S}(\pi, \mathfrak{p})$, and $\gamma$ is a $W_{\mathfrak{p}}$-invariant weight where each element of $\mathbf{R}_{i}$ appears $n-i$ times. Thus, the coordinates of $\gamma+\rho_{\mathfrak{p}}$ are the concatenations of the $c$-blocks for the different $c \in \mathbf{R}_{i}$ for all $i$, ordered by the value of $c$. The longest left coset property says that every pair of elements of the same $c$-block must be reversed in order. That is, they must be in decreasing order in rows (that is, they must satisfy the overshadowing condition) or in the same row. On the other hand, if they are in the same row, the shortest right coset condition assures that they must have remained in the same order, contradicting the longest left coset property. Thus, this tableau must be overshadowing.

Conversely, if a tableau is overshadowing, then the division into $c$-blocks fixes a unique parabolic-singular permutation which sends $\gamma+\rho_{\mathfrak{p}}$ to a row reading of this tableau which matches the $c$-blocks of the tableaux $c$-blocks of $\gamma+\rho_{\mathfrak{p}}$ while ordering each row by the order on $c$-blocks in $\gamma+\rho_{\mathfrak{p}}$. This makes the shortest right coset property clear, and the longest left coset property follows because overshadowing shows that every $c$-block is completely reversed.

Let $\mathbb{B}(\lambda)$ be the crystal associated to an irreducible representation of $\mathfrak{g}$ of highest weight $\lambda$. By [KTWWY19a, Proposition 2.9], the crystal $\mathcal{B}(\widetilde{\mathbf{R}})$ is isomorphic to $\mathbb{B}\left(t_{1} \varpi_{1}\right) \otimes \cdots \otimes \mathbb{B}\left(t_{q} \varpi_{1}\right)$, where $\widetilde{\mathbf{R}}=\left\{c_{1}^{t_{1}}, \ldots, c_{q}^{t_{q}}\right\}$.

Now, we shall describe the inclusion $\mathcal{B}(\mathbf{R}) \subset \mathcal{B}(\widetilde{\mathbf{R}})$. First, consider the case when $\lambda$ is fundamental. The elements of $\mathcal{B}\left(y_{i, c}\right)$ are in bijection with partitions fitting inside an $i \times n-i$ box, that is, with no more than $i$ parts and $\xi_{p} \leq n-i$ (cf. Section 2.5.3). We identify a partition with its diagram $\left\{(a, b) \in \mathbb{Z}_{>0} \times \mathbb{Z}_{>0} \mid 1 \leq a \leq \xi_{b}\right\}$, 
and to the partition $\xi$ we associate the monomial

$$
y_{\xi, c}=y_{i, c} \cdot \prod_{(a, b) \in \xi} z_{i-a+b, c-a-b}^{-1} .
$$

Thus, we wish to factor these into terms corresponding to $\mathcal{B}\left(y_{1, c+j}\right)$ for $j=-i+$ $1, \ldots, i-1$. This is easily done using the formula

$$
y_{i, c}=y_{1,-i+1} y_{1,-i+3} \cdots y_{1, i-1} \prod_{k=1}^{i-1} \prod_{j=0}^{k-1} z_{i-k, 2 j-k}^{-1} .
$$

Thus, we have that

$$
y_{\xi, c}=\prod_{p=1}^{i} y_{1, c+i-2 p+1} \prod_{q=1}^{\xi_{p}+i-p} z_{q, c-q+i-2 p}^{-1}=\prod_{p=1}^{i} y_{\left(\xi_{p}+i-p\right), c+i+1-2 p},
$$

where we consider $\left(\xi_{p}+i-p\right)$ as a partition with one row. This gives us an element in $\mathcal{B}\left(y_{1, c+i+1-2 p}\right)$, resulting in the inclusion

$$
\mathcal{B}\left(y_{i, c}\right) \subset \prod_{j=-i+1, \ldots, i-1} \mathcal{B}\left(y_{1, c+j}\right) .
$$

For general $\lambda$ we take the product over all such inclusions. More precisely, for $p \in \mathcal{B}(\mathbf{R})$ we write $p=\prod_{i \in I, c \in \mathbf{R}_{i}} y_{\xi^{n-i, c}, c}$. Then by the above argument we can view $y_{\xi^{i, c}, c} \in \prod_{j=-n+i+1, \ldots, n-i-1} \mathcal{B}\left(y_{1, c+j}\right)$, and hence

$$
p \in \prod_{i \in I, c \in \mathbf{R}_{i}} \prod_{j=-n+i+1, \ldots, n-i-1} \mathcal{B}\left(y_{1, c+j}\right)=\mathcal{B}(\widetilde{\mathbf{R}}) .
$$

This procedure has a nice description in terms of diagrams. Consider a monomial $p \in \mathcal{B}(\mathbf{R})$. Recall that $p$ can be represented diagrammatically as in Section 2.5.3. where here we assume that $\mathbf{R}$ is an integral set of parameters. To define the image of $p$ in $\mathcal{B}(\widetilde{\mathbf{R}})$, the idea is to "project" the circled vertices onto the line corresponding to the $n-1$ node of the Dynkin diagram and fill the squares along this projection with 1's.

For instance, if we work in type $A_{6}$, with $\mathbf{R}_{3}=\{4\}$, all other $\mathbf{R}_{i}$ empty, and we attach the partition $(2,1)$ to this vertex, then we have the picture on the left. After projecting we obtain the picture on the right:
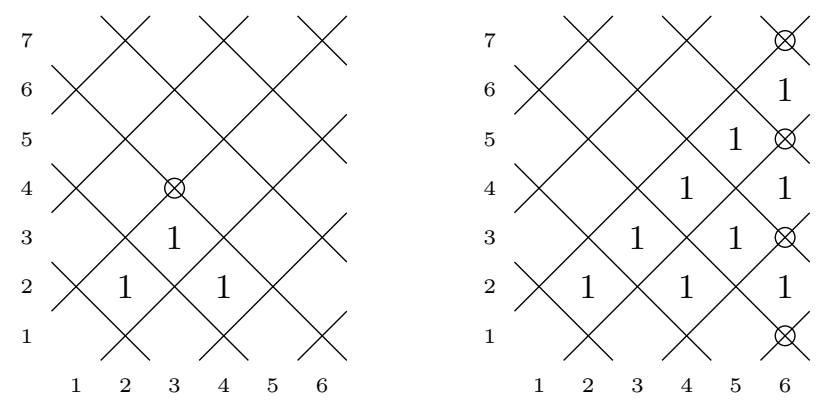

In general, the inclusion $\mathcal{B}(\mathbf{R}) \rightarrow \mathcal{B}(\widetilde{\mathbf{R}})$ is defined by applying this projection to every vertex. For instance, consider the monomial data $p_{0} \in \mathcal{B}(\mathbf{R})$ on the left below, 
where $\mathbf{R}_{3}=\mathbf{R}_{5}=\{4\}$ and all other $\mathbf{R}_{i}$ are empty. The corresponding monomial data in $\mathcal{B}(\widetilde{\mathbf{R}})$ is on the right:
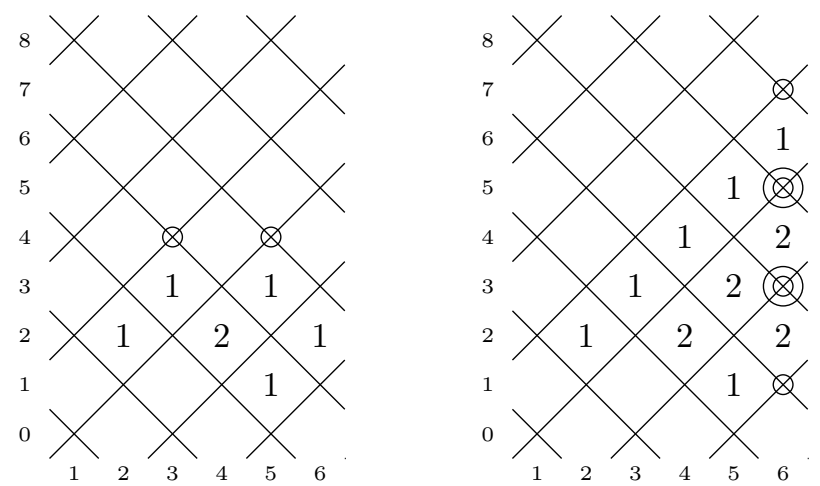

Finally we are ready to prove:

Proposition 4.15. Under the bijection of Proposition 4.13 , Row $_{\widetilde{\mathbf{R}}}(\pi)^{\circ}$ is identified with $\mathcal{B}(\mathbf{R})$.

This completes the proof of part (b) of Theorem 4.3 .

Proof. By the above discussion, we view $\mathcal{B}(\mathbf{R}) \subset \mathcal{B}(\widetilde{\mathbf{R}})$. Let $\mathbf{S}=\left(\mathbf{S}_{i}\right) \in \mathcal{B}(\mathbf{R})$, and suppose it corresponds to $T \in \operatorname{Row}_{\widetilde{\mathbf{R}}}(\pi)$ under the bijection of Proposition 4.13 . Denoting the rows of $T$ by $T_{i}$, we have that $T_{n}=\widetilde{\mathbf{R}} \backslash\left(\mathbf{S}_{n-1}+1\right), T_{1}=\mathbf{S}_{1}+n$, and for $i=1, \ldots, n-2$,

$$
T_{i}=\left(\mathbf{S}_{i}+(n-i)\right) \backslash\left(\mathbf{S}_{i-1}+(n-i+1)\right) .
$$

We'll show that $T \in \operatorname{Row}_{\widetilde{\mathbf{R}}}(\pi)^{\circ}$; i.e., $T$ has an $\mathbf{R}$-coloring. We'll first show that it suffices to prove this in the case when $p$ consists of only one vertex (i.e., $|\mathbf{R}|=1$ ). Without loss of generality assume that $\mathbf{R}$ is integral. Now color each partition in $p$. For instance we could have the example on the left below. When we view $p$ as a monomial datum in $\mathcal{B}\left(N \varpi_{1}, \widetilde{\mathbf{R}}\right)$ we remember the color of the partitions. In the example we obtain the diagram on the right.
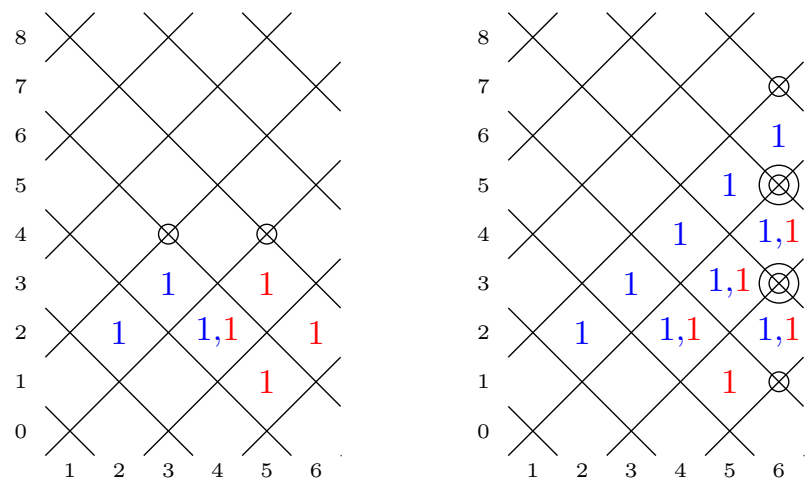

Now, when we apply the bijection, we naturally obtain a row tableau whose entries are colored (we don't know a priori that this is an $\mathbf{R}$-colored tableau - this is what we want to show). Indeed when we look at $\left(\mathbf{S}_{i}+(n-i)\right) \backslash\left(\mathbf{S}_{i-1}+(n-i+1)\right)$, we preserve the color of the elements that haven't been cancelled (for $c \in \mathbf{S}_{i}$, the 
element $c+n-i \in \mathbf{S}_{i}+(n-i)$ is understood to have the same color as $\left.c\right)$. Moreover, the last row is given by $\widetilde{\mathbf{R}} \backslash\left(\mathbf{S}_{n-1}+1\right)$, and the elements of $\widetilde{\mathbf{R}}$ are colored the same color as the node which "overshadowed" them. This is much easier with an example: the bijection applied to the above monomial data results in the following colored row tableau (which happens to contain two empty rows):

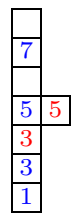

Note that the red content is precisely the block corresponding to $4 \in \mathbf{S}_{5}$, and the blue content is the block corresponding to $4 \in \mathbf{S}_{3}$. Moreover if $p$ consisted of, say, just the red partition, then the resulting row tableau is the red part of the above tableau. This shows that it suffices to consider the case where $|\mathbf{R}|=1$ and show that the resulting row tableau is overshadowing.

To this end, suppose $\mathbf{R}_{n-i}=\{k\}$ (so the other multisets $\mathbf{R}_{j}$ are empty) and in the monomial data $p$ the partition $\lambda=\left(\lambda_{1} \geq \cdots \geq \lambda_{i} \geq 0\right)$ corresponds to $k$. Then for $j=1, \ldots, i, T$ has content $k+i-2 j+1$ going down the rows, which is manifestly overshadowing. This proves that $T \in \operatorname{Row}_{\widetilde{\mathbf{R}}}(\pi)^{\circ}$ for any $p \in \mathcal{B}(\mathbf{R})$.

To prove that the bijection $\mathcal{B}(\mathbf{R}) \rightarrow \operatorname{Row}_{\widetilde{\mathbf{R}}}(\pi)^{\circ}$ is surjective, given $T \in \operatorname{Row}_{\widetilde{\mathbf{R}}}(\pi)^{\circ}$, choose an $\mathbf{R}$-coloring of $T$. This partitions the contents of $T$ into $c$-blocks, and for each such block we can reverse the process above to construct a monomial datum. If we do this for all blocks at once we obtain a datum in $\mathcal{B}(\mathbf{R}) \subset \mathcal{B}(\widetilde{\mathbf{R}})$.

Remark 4.16. Under this bijection, we obtain a crystal structure on overshadowing tableaux. One can easily work out that this coincides with the one induced by Brundan and Kleshchev's crystal structure on row tableaux in BK08, Section 4.3].

\section{Proof of Theorem 4.3(D): The Classical limit}

In this section, we will study the classical limit of our isomorphism

$$
\Phi: Y_{\mu}^{\lambda}(\mathbf{R}) \stackrel{\sim}{\longrightarrow} W(\pi, \mathfrak{p})_{\mathbf{R}} .
$$

Our goal is to establish part (d) of Theorem 4.3 and show that this classical limit agrees with the Mirković-Vybornov isomorphism.

Remark 5.1. We may immediately save ourselves some work with an observation: it suffices to prove the case of $\lambda=N \varpi_{1}$, as in general both isomorphisms are defined by restricting this case to closed subvarieties.

5.1. More about slices to nilpotent orbits. In this subsection we let $G$ be a reductive algebraic group over $\mathbb{C}$, with Lie algebra $\mathfrak{g}$. We will fix throughout a nonzero nilpotent element $e \in \mathfrak{g}$ and an $\mathfrak{s l}_{2}$-triple $\{e, h, f\}$. In this section, we slightly generalize some of the results on Slodowy slices from [GG02, showing in particular that the classical Slodowy slice and the transverse slice considered in MV07a are Poisson isomorphic. Since these results may be of independent interest, we provide brief proofs.

Definition 5.2. Let $C \subset \mathfrak{g}$ be an $\operatorname{ad}_{h}$-invariant subspace such that $\mathfrak{g}=[\mathfrak{g}, e] \oplus C$. Then the affine space $\mathcal{M}=e+C$ is called an $\mathbf{M V}$ slice. 
The most natural choice of such a slice is the Slodowy slice, where $C=\mathfrak{g}^{f}$ (cf. Section 3.1). There are many others however.

Remark 5.3. An MV slice $\mathcal{M}=e+C$ is a transverse slice to the nilpotent orbit $\mathbb{O}_{e}$ at the point $e$.

From now on, we assume that $\mathcal{M}$ is an MV slice. Note that the eigenvalues of $\operatorname{ad}_{h}$ acting on $C$ are necessarily non-positive. From our $\mathfrak{s l}_{2}$-triple we get a homomorphism $\mathrm{SL}_{2} \rightarrow G$, and we will denote by $\gamma(t)$ the image of $\left(\begin{array}{cc}t & 0 \\ 0 & t^{-1}\end{array}\right)$ in $G$. We consider the $\mathbb{C}^{\times}$-action (the Kazhdan action) on $\mathfrak{g}$ defined by

$$
\rho(t) \cdot x=t^{2}\left(\operatorname{Ad} \gamma\left(t^{-1}\right)\right)(x) .
$$

Note that $\rho$ preserves $\mathcal{M}$ and contracts it to the unique fixed point $e$.

Consider the decomposition $\mathfrak{g}=\bigoplus_{i \in \mathbb{Z}} \mathfrak{g}_{i}$ into $\operatorname{ad}_{h}$ weight spaces. As in Section 3.1 there is a non-degenerate skew-symmetric form $\langle x, y\rangle=(e,[x, y])$ on $\mathfrak{g}_{-1}$. Choose a Lagrangian subspace $\mathfrak{l} \subset \mathfrak{g}_{-1}$ with respect to $\langle\cdot, \cdot \cdot\rangle$.

Define the nilpotent Lie subalgebra $\mathfrak{m}=\mathfrak{l} \oplus \bigoplus_{i \leq-2} \mathfrak{g}_{i}$ and the corresponding unipotent subgroup $M \subset G$. Note that $\mathfrak{m}^{\perp}=[e, \mathfrak{l}] \oplus \bigoplus_{i<0} \mathfrak{g}_{i}$ is the orthogonal complement of $\mathfrak{m}$ with respect to the Killing form. The following result is a generalization of Lemma 2.1 in GG02.

Lemma 5.4. The adjoint action map $\alpha: M \times \mathcal{M} \rightarrow e+\mathfrak{m}^{\perp}$ is a $\mathbb{C}^{\times}$-equivariant isomorphism of affine varieties. Here $\mathbb{C}^{\times}$acts on $e+\mathfrak{m}^{\perp}$ by $\rho$ and on $M \times \mathcal{M}$ by

$$
t \cdot(g, x)=\left(\gamma\left(t^{-1}\right) g \gamma(t), \rho(t) \cdot x\right) .
$$

Proof. Since $\mathcal{M}$ is an MV slice we have $C \subset \bigoplus_{i \leq 0} \mathfrak{g}(i) \subset \mathfrak{m}^{\perp}$, so indeed the image of the adjoint map $M \times \mathcal{M} \rightarrow \mathfrak{g}$ is contained in $\bar{e}+\mathfrak{m}^{\perp}$.

Next, since $\mathfrak{g}=[\mathfrak{g}, e] \oplus C$ it follows that $[\mathfrak{m}, e] \cap C=0$. We also have $\operatorname{dim} \operatorname{Ker}(\operatorname{ad} f)$ $=\operatorname{dim} C$, since both spaces are complementary to $[\mathfrak{g}, e]$ in $\mathfrak{g}$. Since ade $: \mathfrak{m} \rightarrow[e, \mathfrak{m}]$ is an isomorphism,

$$
\begin{aligned}
\operatorname{dim} \mathfrak{m}^{\perp} & =\operatorname{dim} \mathfrak{m}+\operatorname{dim} \mathfrak{g}(0)+\operatorname{dim} \mathfrak{g}(-1)=\operatorname{dim}[\mathfrak{m}, e]+\operatorname{dim} \operatorname{Ker}(\operatorname{ad} f) \\
& =\operatorname{dim}[\mathfrak{m}, e]+\operatorname{dim} C .
\end{aligned}
$$

So $\mathfrak{m}^{\perp}=[\mathfrak{m}, e] \oplus C$. The remainder of the proof proceeds as in GG02].

Following Section 3.2 in GG02: $e$ is a regular value for the moment map $\mu$ : $\mathfrak{g}^{*} \rightarrow \mathfrak{m}^{*}, \mu^{-1}(e)=e+\mathfrak{m}^{\perp}$ (under $\left.\mathfrak{g}^{*} \cong \mathfrak{g}\right)$, and it follows from Lemma 5.4 that we have a Hamiltonian reduction of the Poisson structure on $\mathfrak{g}^{*}$ to $\mathcal{M}$. It is induced from the isomorphisms

$$
\mathcal{M} \cong \mu^{-1}(e) / M, \quad \mathbb{C}[\mathcal{M}] \cong\left(\mathbb{C}[\mathfrak{g}] / I\left(\mu^{-1}(e)\right)\right)^{M} .
$$

Theorem 5.5. There is a $\mathbb{C}^{\times}$-equivariant isomorphism of affine Poisson varieties between any two $M V$ slices.

Proof. With $\mathfrak{l}$ and $\mathfrak{m}$ fixed as above, for MV slices $\mathcal{M}_{1}, \mathcal{M}_{2}$ we have $\mathbb{C}^{\times}$-equivariant isomorphisms

$$
M \times \mathcal{M}_{1} \cong e+\mathfrak{m}^{\perp} \cong M \times \mathcal{M}_{2}
$$

by Lemma 5.4. The Poisson structures on $\mathcal{M}_{1}, \mathcal{M}_{2}$ are both induced by Hamiltonian reduction, giving us the desired Poisson isomorphism. 
Remark 5.6. As in GG02, Section 3.1], any MV slice $\mathcal{M}$ also inherits an induced Poisson structure as a subvariety of $\mathfrak{g}$ (with its Kostant-Kirillov-Souriau Poisson structure under $\mathfrak{g} \cong \mathfrak{g}^{*}$ ) by applying [Vai94, Proposition 3.10]. This Poisson structure agrees with that given above via Hamiltonian reduction; cf. GG02, Section $3.2]$.

5.2. More about affine Grassmannian slices. Let us briefly recall some aspects of the "loop group" description of the slices $\mathrm{Gr}_{\mu}^{\bar{\lambda}}$ in the affine Grassmannian of $G=\mathrm{SL}_{n}$ to supplement the lattice description given in Section 2.1.

Remark 5.7. In Section 2.1 we defined the slices in the affine Grassmannian of $P G L_{n}$. Here use $G=\mathrm{SL}_{n}$; this doesn't make a difference. In fact, we can use any group whose Lie algebra is $\mathfrak{s l}_{n}$ [KWWY14, Section 2G].

$\mathrm{Gr}_{\mu}^{\bar{\lambda}}$ is a transverse slice to $\mathrm{Gr}^{\mu} \subset \overline{\mathrm{Gr}^{\lambda}}$, defined as the intersection

$$
\mathrm{Gr}_{\mu}^{\bar{\lambda}}=\mathrm{Gr}_{\mu} \cap \overline{\mathrm{Gr}^{\lambda}}
$$

where $\mathrm{Gr}_{\mu}=G_{1}\left[t^{-1}\right] t^{w_{0} \mu}$ is an orbit for the opposite group $G_{1}\left[t^{-1}\right]=\operatorname{Ker}\left(G\left[t^{-1}\right] \stackrel{t \mapsto \infty}{\rightarrow}\right.$ $G)$. Every point in $\mathrm{Gr}_{\mu}$ has a unique representative of the form $g t^{w_{0} \mu}$, where $g=\left(a_{i j}\right) \in G_{1}\left[t^{-1}\right]$ satisfies

$$
a_{i j}=\delta_{i j}+a_{i j}^{(1)} t^{-1}+a_{i j}^{(2)} t^{-2}+\cdots+\in \delta_{i j}+t^{p_{i}-p_{j}-1} \mathbb{C}\left[t^{-1}\right] .
$$

(Recall that we are denoting $w_{0} \mu=\left(p_{1}, \ldots, p_{n}\right)$.) In this way, $\mathrm{Gr}_{\mu}^{\bar{\lambda}}$ may be considered as a closed subscheme of $G_{1}\left[t^{-1}\right]$.

Remark 5.8. It is sometimes convenient to work with the group $G_{1}\left[\left[t^{-1}\right]\right]$. One advantage is that elements of this group admit Gauss decompositions,

$$
G_{1}\left[\left[t^{-1}\right]\right]=U_{1}^{-}\left[\left[t^{-1}\right]\right] T_{1}\left[\left[t^{-1}\right]\right] U_{1}^{+}\left[\left[t^{-1}\right]\right],
$$

where $U^{ \pm}, T \subset G$ are the subgroups of upper/lower triangular and diagonal matrices. The varieties $\operatorname{Gr}_{\mu}^{\bar{\lambda}}$ may also be considered as closed subschemes of $G_{1}\left[\left[t^{-1}\right]\right]$.

In particular, we may describe $\operatorname{Gr}_{\mu}^{\overline{N \varpi 1}}$ as the variety of matrices $g=\left(a_{i j}\right)$ of the form (5.2) (with det $g=1$ ), with the additional constraint that $a_{i j}^{(r)}=0$ for $r>p_{j}$. Then explicitly $g$ corresponds to the lattice

$$
\Lambda=\operatorname{span}_{\mathcal{O}}\left\{t_{j}^{p} e_{j}+\sum_{i, r} a_{i j}^{(r)} t^{p_{j}-r} e_{i}: 1 \leq j \leq n\right\},
$$

allowing us to compare with our previous description (2.1) of $\mathrm{Gr}_{\mu}^{\overline{N \varpi_{1}}}$.

Using the above identification of $\mathrm{Gr}_{\mu}^{\bar{\lambda}} \subset G_{1}\left[\left[t^{-1}\right]\right]$, we now recall how the classical limit of $Y_{\mu}^{\lambda}(\mathbf{R})$ is identified with functions on $\mathrm{Gr}_{\mu}^{\bar{\lambda}}$ (as was promised in Section 2.3). Following Theorems 3.9, 3.12, and Proposition 4.3 in [WWY14,

$$
\begin{aligned}
& A_{i}^{(r)} \mapsto\left[t^{-r}\right] \Delta_{\{1, \ldots, i\},\{1, \ldots, i\}}, \\
& E_{i}^{(r)} \mapsto\left[t^{-r}\right] \frac{\Delta_{\{1, \ldots, i-1, i+1\},\{1, \ldots, i\}}}{\Delta_{\{1, \ldots, i\},\{1, \ldots, i\}}}, \\
& F_{i}^{(s)} \mapsto\left[t^{-s}\right] \frac{\Delta_{\{1, \ldots, i\},\{1, \ldots, i-1, i+1\}}}{\Delta_{\{1, \ldots, i\},\{1, \ldots, i\}}} .
\end{aligned}
$$


Here, for $I, J \subset\{1, \ldots, n\}$ we denote by $\Delta_{I, J}$ the minor with rows $I$ and columns $J$, thought of as a map $G_{1}\left[\left[t^{-1}\right]\right] \rightarrow \mathbb{C}\left[\left[t^{-1}\right]\right]$. Meanwhile, $\left[t^{-r}\right]$ extracts the coefficient of $t^{-r}$. Restricting these functions to the closed subscheme $\operatorname{Gr}_{\mu}^{\bar{\lambda}} \subset G_{1}\left[\left[t^{-1}\right]\right]$ gives the desired isomorphism.

The following result is clear from the structure of the GKLO representation KWWY14, Theorem 4.5]. It also follows from similar results for Zastava spaces

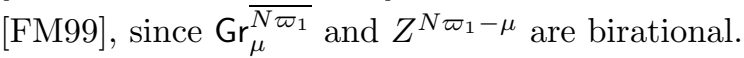

Proposition 5.9. The functions $A_{i}^{(r)}, E_{i}^{(r)}$ for $i \in I, 1 \leq r \leq m_{i}$, are birational coordinates on $\mathrm{Gr}_{\mu}^{\bar{\lambda}}$.

5.3. The $\mathrm{MV}$ isomorphism. As per usual, now fix $\lambda \geq \mu$ dominant coweights and associated partitions $\tau \geq \pi$ of $N$.

Let $e_{\pi} \in \mathfrak{g l}_{N}$ be the nilpotent element with lower triangular Jordan type $\pi=$ $\left(p_{1} \leq \cdots \leq p_{n}\right) \vdash N$. We will consider the transpose MV slice

$$
\mathcal{T}_{\pi}=\left\{\begin{array}{ll} 
& \text { (a) } X_{i j} \text { has size } p_{i} \times p_{j} \\
& \text { (b) } X_{i i} \text { has 1's below diagonal entries in final } \\
& \text { column, } \\
& \text { (c) } X_{i j} \text { for } i \neq j \text { has entries in final column, } \\
& \text { but not below row } p_{j} .
\end{array}\right\}
$$

Recall the description of $\mathrm{Gr}_{\mu}^{\bar{\lambda}}$ from Section 2.1. With this description and the definition of $\mathcal{T}_{\pi}$ in mind, we can now give a slightly more precise formulation of the MV isomorphism Theorem 4.1.

For any $\Lambda \in \mathrm{Gr}_{\mu}^{\bar{\lambda}}$, identify $\Lambda_{0} / \Lambda \cong \mathbb{C}^{N}$ via the basis $E_{\pi}$; in particular we may identify multiplication by $t$ on $\Lambda_{0} / \Lambda$ with an element $X \in \mathfrak{g l}_{N}$. The map taking $\Lambda$ to $X \in \mathfrak{g l}_{N}$ defines an isomorphism $\mathrm{Gr}_{\mu}^{\bar{\lambda}} \stackrel{\sim}{\longrightarrow} \mathcal{T}_{\pi} \cap \overline{\mathbb{O}_{\tau}}$. It is compatible with the inclusions of closed subvarieties $\operatorname{Gr}_{\mu}^{\bar{\lambda}} \subset \mathrm{Gr}_{\mu}^{\overline{N \varpi_{1}}}$ and $\mathcal{T}_{\pi} \cap \overline{\mathbb{O}_{\tau}} \subset \mathcal{T}_{\pi} \cap \mathcal{N}_{\mathfrak{g l}_{N}}$.

Following [CK18, Section 3.3], it is straightforward to write the above isomorphism explicitly in coordinates. On the affine Grassmannian side, we identify $\Lambda$ with $g \in G_{1}\left[\left[t^{-1}\right]\right]$ as in the previous section and use the coefficients $a_{i j}^{(r)}$ of the matix entries of $g$ as coordinates. On the nilpotent cone side, the image is a block matrix $X=\left(X_{i j}\right)$. Then under the above isomorphism, the block $X_{i j}$ has interesting entries only in its final column:

$$
X_{i j}=\left(\begin{array}{ccccccc}
0 & 0 & \cdots & 0 & \cdots & 0 & -a_{i j}^{\left(p_{j}\right)} \\
\delta_{i j} & 0 & \cdots & 0 & \ddots & \vdots & \vdots \\
0 & \delta_{i j} & \ddots & & \ddots & 0 & -a_{i j}^{(1)} \\
0 & 0 & \ddots & \ddots & & 0 & 0 \\
0 & \ddots & \ddots & \ddots & \ddots & \vdots & \vdots \\
\vdots & \ddots & \ddots & 0 & \delta_{i j} & 0 & 0 \\
0 & \cdots & 0 & 0 & 0 & \delta_{i j} & 0
\end{array}\right) .
$$

5.4. Completing the proof of Theorem 4.3. To finish the proof of the theorem, we will now compare the classical limit of our isomorphism $\Phi: Y_{\mu}^{N \varpi_{1}}(\mathbf{R}) \stackrel{\sim}{\longrightarrow}$ 
$W(\pi)_{\mathbf{R}}$ with the Mirković-Vybornov isomorphism. We interpret the classical limit of $\Phi$ as an isomorphism of coordinate rings

$$
\Phi: \mathbb{C}\left[\mathrm{Gr}_{\mu}^{\overline{N \varpi_{1}}}\right] \stackrel{\sim}{\longrightarrow} \mathbb{C}\left[\mathcal{T}_{\pi} \cap \mathcal{N}_{\mathfrak{g l}_{N}}\right] .
$$

In the notation of the previous section, suppose that $g \in \mathrm{Gr}_{\mu}^{\overline{N \varpi_{1}}} \subset G_{1}\left[\left[t^{-1}\right]\right]$ maps to $X \in \mathcal{T}_{\pi} \cap \mathcal{N}_{\mathfrak{g l}_{N}}$ under the Mirković-Vybornov isomorphism (both being closed points). To complete the proof of the theorem, it is sufficient to prove that

$$
f(g)=\Phi(f)(X) \quad \forall f \in \mathbb{C}\left[\mathrm{Gr}_{\mu}^{\overline{N \varpi_{1}}}\right] .
$$

Remark 5.10. Both sides are irreducible algebraic varieties, so in fact it is sufficient to prove that this equation holds for $f$ ranging over the birational coordinates described in Proposition 5.9.

The isomorphism $\operatorname{gr} Y_{\mu}^{\overline{N \varpi_{1}}}(\mathbf{R}) \cong \mathbb{C}\left[\mathrm{Gr}_{\mu}^{\overline{N \varpi_{1}}}\right]$ was described explicitly in Section 5.2. We now recall Brundan and Kleshchev's identification of the classical limit of $W(\pi)$ with functions on $\mathcal{T}_{\pi}$, following [BK08, Sections 3.3-3.4]. More precisely, they give an explicit isomorphism

$$
W(\pi) \stackrel{\sim}{\longrightarrow} U(\mathfrak{p})^{\mathfrak{m}} \subset U\left(\mathfrak{g l}_{N}\right) .
$$

In the classical limit, we identify $S\left(\mathfrak{g l}_{N}\right) \cong \mathbb{C}\left[\mathfrak{g l}_{N}\right]$ via the trace pairing and $S(\mathfrak{p})^{\mathfrak{m}} \cong$ $\mathbb{C}\left[e_{\pi}+\mathfrak{m}^{\perp}\right]^{M}$ (see [BK06, Section 8] for details). Since $\mathcal{T}_{\pi}$ is an MV slice, by Lemma 5.4, there is an isomorphism

$$
\mathbb{C}\left[e+\mathfrak{m}^{\perp}\right]^{M} \stackrel{\sim}{\rightarrow} \mathbb{C}\left[\mathcal{T}_{\pi}\right]
$$

by restriction. In other words, the isomorphism gr $W(\pi) \cong \mathbb{C}\left[\mathcal{T}_{\pi}\right]$ comes by the composition

$$
\operatorname{gr} W(\pi) \hookrightarrow \mathbb{C}\left[\mathfrak{g l}_{N}\right] \rightarrow \mathbb{C}\left[\mathcal{T}_{\pi}\right]
$$

where the first arrow is Brundan-Kleshchev's embedding and the second is restriction.

Brundan-Kleshchev's embedding is defined via explicit elements $T_{i j ; 0}^{(r)} \in U\left(\mathfrak{g l}_{N}\right)$, defined in [BK06, Section 9] (see also [BK08, Section 3.3]). Forming the $n \times n-$ matrix $T(u)=\left(T_{i j}(u)\right)$ whose entries are formal series of functions $T_{i j}(u)=\delta_{i j}+$ $\sum_{r>0} T_{i j ; 0}^{(r)} u^{-r}$, we take its formal Gauss decomposition

$$
T(u)=F(u) D(u) E(u)
$$

where $D(u)$ is diagonal and $E(u)$ (resp., $F(u)$ ) is upper (resp., lower) unitriangular. Denote the diagonal entries of $D(u)$ by $D_{i}(u)=1+\sum_{r>0} D_{i}^{(r)} u^{-r}$ and the superdiagonal entries of $E(u)$ by $E_{i}(u)=\sum_{r>0} E_{i}^{(r)} u^{-r}$. Then the elements $D_{i}^{(r)}, E_{i}^{(r)} \in$ $U\left(\mathfrak{g l}_{N}\right)$ are the images of the same-named elements of $W(\pi)$ (and similarly for the $\left.F_{i}^{(s)}\right)$.

By abuse of notation, let us denote by $T_{i j ; 0}^{(r)}, D_{i}^{(r)}$, etc., the corresponding elements of the associated graded algebras.

Lemma 5.11. Suppose $\mathrm{Gr}_{\mu}^{\overline{N \varpi_{1}}} \ni g \mapsto X \in \mathcal{T}_{\pi} \cap \mathcal{N}_{\mathfrak{g l}_{N}}$ are closed points corresponding under the Mirković-Vybornov isomorphism. Then

$$
T_{i, j ; 0}^{(r)}(X)=(-1)^{r} a_{j i}^{(r)} .
$$


Proof. Recall our conventions on the pyramid $\pi$ from Section 3.1.1. By definition,

$$
T_{i j ; 0}^{(r)}=\sum_{s=1}^{r}(-1)^{r-s} \sum_{\substack{k_{1}, \ldots, k_{s} \\ \ell_{1}, \ldots, \ell_{s}}} e_{k_{1}, \ell_{1}} \cdots e_{k_{s}, \ell_{s}},
$$

where the sum is over sequences with $1 \leq k_{t}, \ell_{t} \leq N$, satisfying conditions (a), (b), (c), (e), and (f) from [BK08, Section 3.3]. In the classical limit, this is a function on $\mathfrak{g l}_{N}$ via the trace pairing. When restricted to $\mathcal{T}_{\pi}$, conditions (a), (b), (e), and (f) imply that the value at $X$ has the form

$$
\sum_{s=1}^{r}(-1)^{r-s} \sum_{\substack{x_{2}, \ldots, x_{s} \\ r_{1}+\cdots+r_{s}=r}}(-1)^{s} a_{j x_{2}}^{\left(r_{1}\right)} a_{x_{2} x_{3}}^{\left(r_{2}\right)} \cdots a_{x_{s} i}^{\left(r_{s}\right)},
$$

where the sum is over all sequences where $1 \leq x_{t} \leq n$. However, condition (c) implies that only the term with $s=1$ contributes. This proves the claim.

Now, from [4.3) and Lemma4.9] it follows that the classical limit of $\Phi: Y_{\mu}^{N \varpi_{1}}(\mathbf{R})$ $\stackrel{\sim}{\rightarrow} W(\pi)_{\mathbf{R}}$ sends

$$
A_{i}^{(r)} \mapsto(-1)^{r} Q_{i}^{(r)}, \quad E_{i}^{(r)} \mapsto(-1)^{r} E_{i}^{(r)},
$$

where $Q_{i}(u)=D_{1}(u) \cdots D_{i}(u)$. Therefore, by Remark 5.10 the following result completes the proof of the main theorem:

Proposition 5.12. With notation as in the previous lemma, suppose that $g \mapsto X$. Then for $i=1, \ldots, n-1$ we have equality of evaluations

$$
\begin{aligned}
& A_{i}^{(r)}(g)=(-1)^{r} Q_{i}^{(r)}(X), \\
& E_{i}^{(r)}(g)=(-1)^{r} E_{i}^{(r)}(X)
\end{aligned}
$$

for the functions $A_{i}^{(r)}, E_{i}^{(r)} \in \mathbb{C}\left[\mathrm{Gr}_{\mu}^{\overline{N \varpi_{1}}}\right]$ and $D_{i}^{(r)}, E_{i}^{(r)} \in \mathbb{C}\left[\mathcal{T}_{\pi} \cap \mathcal{N}_{\mathfrak{g l}_{N}}\right]$, respectively.

Proof. If we take the Gauss decomposition of $T(u)$ and then evaluate the result at the point $X$, we will get the same result as first evaluating $T(u)$ at $X$ and then taking Gauss decomposition.

By the previous lemma, if we evaluate $T(u)$ at $X$ we get the matrix $g(-u)^{T}$ (i.e., the transpose of $g=g(t) \in G_{1}\left[\left[t^{-1}\right]\right]$ evaluated at $t=-u$ ). Using the relation between the minors of a matrix and its transpose, we observe that

$$
\begin{aligned}
E_{i}^{(r)}(g) & =\left[t^{-r}\right] \frac{\Delta_{\{1, \ldots, i-1, i+1\},\{1, \ldots, i\}}}{\Delta_{\{1, \ldots, i\},\{1, \ldots, i\}}}(g(t)) \\
& =\left[t^{-r}\right] \frac{\Delta_{\{1, \ldots, i\},\{1, \ldots, i-1, i+1\}}}{\Delta_{\{1, \ldots, i\},\{1, \ldots, i\}}}\left(g(t)^{T}\right) \\
& =(-1)^{r}\left[u^{-r}\right] \frac{\Delta_{\{1, \ldots, i\},\{1, \ldots, i-1, i+1\}}}{\Delta_{\{1, \ldots, i\},\{1, \ldots, i\}}}\left(g(-u)^{T}\right) .
\end{aligned}
$$

The latter precisely extracts the superdiagonal entries of the " $E$ " part of the Gauss decomposition of $g(-u)^{T}$. Hence $E_{i}^{(r)}(g)=(-1)^{r} E_{i}^{(r)}(X)$, as claimed. A similar calculation applies to $A_{i}^{(r)}$. 


\section{ACKNOWLEDGMENTS}

The authors thank Tomoyuki Arakawa, Joel Kamnitzer, Alex Molev, Stephen Morgan, and Dinakar Muthiah for helpful conversations. We also thank an anonymous referee for very helpful comments. The first and second authors thank the University of Sydney for hosting visits where major parts of this research were conducted and for providing an International Research Collaboration Award to support the first author's visit.

\section{REFERENCES}

[BB81] Alexandre Beulinson and Joseph Bernstein, Localisation de g-modules (French, with English summary), C. R. Acad. Sci. Paris Sér. I Math. 292 (1981), no. 1, 15-18. MR610137

[BB82] Walter Borho and Jean-Luc Brylinski, Differential operators on homogeneous spaces. I. Irreducibility of the associated variety for annihilators of induced modules, Invent. Math. 69 (1982), no. 3, 437-476, DOI 10.1007/BF01389364. MR679767

[BB93] A. Bělinson and J. Bernstein, A proof of Jantzen conjectures, I. M. Gel'fand Seminar, Adv. Soviet Math., vol. 16, Amer. Math. Soc., Providence, RI, 1993, pp. 1-50. MR 1237825

[BB09] Jonathan Brown and Jonathan Brundan, Elementary invariants for centralizers of nilpotent matrices, J. Aust. Math. Soc. 86 (2009), no. 1, 1-15, DOI 10.1017/S1446788708000608. MR2495993

[BF12] Alexander Braverman and Michael Finkelberg, Pursuing the double affine Grassmannian II: Convolution, Adv. Math. 230 (2012), no. 1, 414-432, DOI 10.1016/j.aim.2011.12.007. MR2900549

[BFN] Alexander Braverman, Michael Finkelberg, and Hiraku Nakajima, Coulomb branches of $3 d \mathcal{N}=4$ quiver gauge theories and slices in the affine Grassmannian, with two appendices by Braverman, Finkelberg, Joel Kamnitzer, Ryosuke Kodera, Nakajima, Ben Webster, and Alex Weekes, Adv. Theor. Math. Phys. 23 (2019), no. 1, 75-166, DOI 10.4310/ATMP.2019.v23.n1.a3. MR 4020310

[BFN18] Alexander Braverman, Michael Finkelberg, and Hiraku Nakajima, Towards a mathematical definition of Coulomb branches of 3-dimensional $\mathcal{N}=4$ gauge theories, II, Adv. Theor. Math. Phys. 22 (2018), no. 5, 1071-1147, DOI 10.4310/ATMP.2018.v22.n5.a1. MR.3952347

[BK05] Jonathan Brundan and Alexander Kleshchev, Parabolic presentations of the Yangian $Y\left(\mathfrak{g l}_{n}\right)$, Comm. Math. Phys. 254 (2005), no. 1, 191-220, DOI 10.1007/s00220004-1249-6. MR2116743

[BK06] Jonathan Brundan and Alexander Kleshchev, Shifted Yangians and finite $W$ algebras, Adv. Math. 200 (2006), no. 1, 136-195, DOI 10.1016/j.aim.2004.11.004. MR2199632

[BK08] Jonathan Brundan and Alexander Kleshchev, Representations of shifted Yangians and finite $W$-algebras, Mem. Amer. Math. Soc. 196 (2008), no. 918, viii+107. MR2456464

[BLPW16] Tom Braden, Anthony Licata, Nicholas Proudfoot, and Ben Webster, Quantizations of conical symplectic resolutions II: category $\mathcal{O}$ and symplectic duality (English, with English and French summaries), with an appendix by I. Losev, Astérisque 384 (2016), 75-179. MR3594665

[BO11] Jonathan Brundan and Victor Ostrik, Cohomology of Spaltenstein varieties, Transform. Groups 16 (2011), no. 3, 619-648, DOI 10.1007/s00031-011-9149-2. MR2827037

[CK08] Sabin Cautis and Joel Kamnitzer, Knot homology via derived categories of coherent sheaves. I. The $\mathfrak{s l}(2)$-case, Duke Math. J. 142 (2008), no. 3, 511-588, DOI 10.1215/00127094-2008-012. MR2411561 
[CK18] Sabin Cautis and Joel Kamnitzer, Categorical geometric symmetric Howe duality, Selecta Math. (N.S.) 24 (2018), no. 2, 1593-1631, DOI 10.1007/s00029-017-0362-2. MR.3782430

[CKL10] Sabin Cautis, Joel Kamnitzer, and Anthony Licata, Coherent sheaves and categorical $\mathfrak{s l}_{2}$ actions, Duke Math. J. 154 (2010), no. 1, 135-179, DOI 10.1215/001270942010-035. MR2668555

[FM99] Michael Finkelberg and Ivan Mirković, Semi-infinite flags. I. Case of global curve $\mathbf{P}^{1}$, Differential topology, infinite-dimensional Lie algebras, and applications, Amer. Math. Soc. Transl. Ser. 2, vol. 194, Amer. Math. Soc., Providence, RI, 1999, pp. 81-112, DOI 10.1090/trans2/194/05. MR1729360

[FMO10] Vyacheslav Futorny, Alexander Molev, and Serge Ovsienko, The Gelfand-Kirillov conjecture and Gelfand-Tsetlin modules for finite $W$-algebras, Adv. Math. 223 (2010), no. 3, 773-796, DOI 10.1016/j.aim.2009.08.018. MR2565549

[GG02] Wee Liang Gan and Victor Ginzburg, Quantization of Slodowy slices, Int. Math. Res. Not. 5 (2002), 243-255, DOI 10.1155/S107379280210609X. MR1876934

[GKLO05] A. Gerasimov, S. Kharchev, D. Lebedev, and S. Oblezin, On a class of representations of the Yangian and moduli space of monopoles, Comm. Math. Phys. 260 (2005), no. 3, 511-525, DOI 10.1007/s00220-005-1417-3. MR2182434

[Hes78] Wim H. Hesselink, Polarizations in the classical groups, Math. Z. 160 (1978), no. 3, 217-234, DOI 10.1007/BF01237035. MR480765

[Hik17] Tatsuyuki Hikita, An algebro-geometric realization of the cohomology ring of Hilbert scheme of points in the affine plane, Int. Math. Res. Not. IMRN 8 (2017), 2538-2561, DOI 10.1093/imrn/rnw115. MR3658207

[HTT08] Ryoshi Hotta, Kiyoshi Takeuchi, and Toshiyuki Tanisaki, D-modules, perverse sheaves, and representation theory, translated from the 1995 Japanese edition by Takeuchi, Progress in Mathematics, vol. 236, Birkhäuser Boston, Inc., Boston, MA, 2008. MR2357361

[Kit12] S. N. Kitchen, Cohomology of standard modules on partial flag varieties, Represent. Theory 16 (2012), 317-344, DOI 10.1090/S1088-4165-2012-00419-8. MR2945222

[KMWY18] Joel Kamnitzer, Dinakar Muthiah, Alex Weekes, and Oded Yacobi, Reducedness of affine Grassmannian slices in type A, Proc. Amer. Math. Soc. 146 (2018), no. 2, 861-874, DOI 10.1090/proc/13850. MR3731717

[KTWWY19a] Joel Kamnitzer, Peter Tingley, Ben Webster, Alex Weekes, and Oded Yacobi, Highest weights for truncated shifted Yangians and product monomial crystals, J. Comb. Algebra 3 (2019), no. 3, 237-303, DOI 10.4171/JCA/32. MR4011667

[KTWWY19b] Joel Kamnitzer, Peter Tingley, Ben Webster, Alex Weekes, and Oded Yacobi, On category $\mathcal{O}$ for affine Grassmannian slices and categorified tensor products, Proc. Lond. Math. Soc. (3) 119 (2019), no. 5, 1179-1233, DOI 10.1112/plms.12254. MR3968721

[KWWY14] Joel Kamnitzer, Ben Webster, Alex Weekes, and Oded Yacobi, Yangians and quantizations of slices in the affine Grassmannian, Algebra Number Theory $\mathbf{8}$ (2014), no. 4, 857-893, DOI 10.2140/ant.2014.8.857. MR3248988

[Los10] Ivan Losev, Quantized symplectic actions and $W$-algebras, J. Amer. Math. Soc. 23 (2010), no. 1, 35-59, DOI 10.1090/S0894-0347-09-00648-1. MR2552248

[Los12] Ivan Losev, Isomorphisms of quantizations via quantization of resolutions, Adv. Math. 231 (2012), no. 3-4, 1216-1270, DOI 10.1016/j.aim.2012.06.017. MR2964603

[Maf05] Andrea Maffei, Quiver varieties of type A, Comment. Math. Helv. 80 (2005), no. 1, 1-27, DOI 10.4171/CMH/1. MR2130242

[Mil] Dragan Miličić, Localization and representation theory of reductive Lie groups, http://www.math.utah.edu/ milicic/Eprints/book.pdf.

[MV07a] I. Mirković and K. Vilonen, Geometric Langlands duality and representations of algebraic groups over commutative rings, Ann. of Math. (2) 166 (2007), no. 1, 95-143, DOI 10.4007/annals.2007.166.95. MR2342692

[MV07b] I. Mirković and M. Vybornov, Quiver varieties and Beilinson-Drinfeld Grassmannians of type A, arXiv:0712.4160, 2007. 
[Nak16] Hiraku Nakajima, Towards a mathematical definition of Coulomb branches of 3dimensional $\mathcal{N}=4$ gauge theories, I, Adv. Theor. Math. Phys. 20 (2016), no. 3, 595-669, DOI 10.4310/ATMP.2016.v20.n3.a4. MR3565863

[Vai94] Izu Vaisman, Lectures on the geometry of Poisson manifolds, Progress in Mathematics, vol. 118, Birkhäuser Verlag, Basel, 1994. MR.1269545

[Web] Ben Webster, Gelfand-Tsetlin modules in the Coulomb context, arXiv:1904.05415, 2019.

[Web11] Ben Webster, Singular blocks of parabolic category $\mathcal{O}$ and finite $W$ algebras, J. Pure Appl. Algebra 215 (2011), no. 12, 2797-2804, DOI 10.1016/j.jpaa.2011.03.020. MR2811563

Department of Pure Mathematics, University of Waterloo, Waterloo, Ontario N2L 3G1, Canada; and Perimeter Institute for Theoretical Physics, Waterloo, Ontario N2L 2Y5, CANADA

Email address: ben.webster@uwaterloo.ca

Department of Mathematics, University of British Columbia, Vancouver, British Columbia V6T 1Z4, CANAdA

Email address: weekesal@math.ubc.ca

School of Mathematics and Statistics, University of Sydney, Camperdown, New South Wales 2006, Australia

Email address: oded.yacobi@sydney.edu.au 